\title{
Decoding crystal fractionation in calc-alkaline magmas from the Bezymianny Volcano (Kamchatka, Russia) using mineral and bulk rock compositions
}

\author{
Renat R. Almeev ${ }^{\mathrm{a}, *}$, Jun-Ichi Kimura ${ }^{\mathrm{b}}$, Alexei A. Ariskin ${ }^{\mathrm{c}}$, Alexey Yu. Ozerov ${ }^{\mathrm{d}}$ \\ a Institute of Mineralogy, Leibniz University of Hannover, Callinstr. 3, Hannover, 30167, Germany \\ b Institute for Research on Earth Evolution, JAMSTEC, 2-15 Natsushima-cho, Yokosuka 237-0061, Japan \\ c Vernadsky Institute, Kosygin Str. 19, Moscow, 119991, Russia \\ d Institute of Volcanology and Seismology, Bulvar Piipa, Petropavlovsk-Kamchatsky, 683006, Russia
}

\section{A R T I C L E I N F O}

\section{Article history:}

Received 28 December 2011

Accepted 16 January 2013

Available online 24 January 2013

\section{Keywords:}

Bezymianny Volcano

Fractional crystallization

Magma mixing

Calc-alkaline trend of magma differentiation

Link between HMBs, HABs, andesites and

dacites

\begin{abstract}
A B S T R A C T
We present a new dataset for whole-rock major, trace, isotopic, and phenocryst compositions indicating a genetic link between andesites of the Holocene eruptions of the Bezymianny stratovolcano (the Bezymianny stage), the andesitic to dacitic Late Pleistocene lava dome complex (the pre-Bezymianny stage), and the magnesian to high-alumina basalts of the adjacent Kliuchevskoi Volcano. We demonstrate that volcanic products from the Bezymianny stage of volcano evolution are most likely the products of magma mixing between silicic products of the earliest stages of magma fractionation and the less evolved basaltic andesite parental melts periodically injected into the magma reservoir. In contrast, the intermediate and silicic magmas of the pre-Bezymianny stage together with basalts from Kliuchevskoi much more closely resemble the liquid line of descent and may represent a unique prolonged and continuous calc-alkaline trend of magma evolution from high-magnesian basalt to dacite. As a result of the geothermobarometry, we recognize variable conditions of magma fractionation and magma storage beneath Bezymianny for different magma types during its evolution since the Late Pleistocene: (1) $1100-1150{ }^{\circ} \mathrm{C}, 500-640 \mathrm{MPa}, 1-2.5 \mathrm{wt} . \% \mathrm{H}_{2} \mathrm{O}$ for parental basaltic andesite; (2) $1130-1050{ }^{\circ} \mathrm{C}, 700-600 \mathrm{MPa}, 2.5-5$ wt. $\% \mathrm{H}_{2} \mathrm{O}$ for two-pyroxene andesites; (3) $1040-990{ }^{\circ} \mathrm{C}, 560-470 \mathrm{MPa}$, 5-6.5 wt.\% $\mathrm{H}_{2} \mathrm{O}$ for orthopyroxene-bearing andesites; (4) 950-1000 ${ }^{\circ} \mathrm{C}, 450-150 \mathrm{MPa}$, 3.5-5.5 wt.\% $\mathrm{H}_{2} \mathrm{O}$ for hornblende-bearing andesites; and (5) $950-900{ }^{\circ} \mathrm{C}, 410-250 \mathrm{MPa}, 6-7 \mathrm{wt} . \% \mathrm{H}_{2} \mathrm{O}$ for dacites. Repeated basalt injections and magma fractionation combined with internal mixing in the magma chamber are the main processes responsible for both the complex petrography and the geochemical trends observed in the lavas of Bezymianny Volcano.
\end{abstract}

(c) 2013 Elsevier B.V. All rights reserved.

\section{Introduction}

Calc-alkaline andesites are predominant and characteristic volcanic products of active continental margins and mature island arcs (Gill, 1981; Tatsumi and Takahashi, 2006). Their geochemical characteristics are reminiscent of the Earth's continental crust, so understanding andesite magma genesis may provide key constraints on understanding more general processes, such as those that proceeded during the early stages of silicate Earth differentiation, resulting in the formation and growth of the Earth's crust. Therefore, the formation of andesitic melts is one of the longstanding problems in igneous petrology and it continues to be the major subject of a number of geochemical and experimental investigations.

The key aspects of understanding andesite genesis are identifying the source(s), differentiation mechanism(s), and physicochemical conditions under which intermediate and silicic magmas can be produced.

\footnotetext{
* Corresponding author. Fax: + 495117623045.

E-mail address: r.almeev@mineralogie.uni-hannover.de (R.R. Almeev).
}

A number of hypotheses on andesite genesis concur in the literature and supplement each other, pointing to the complexity of the processes occurring in volcano plumbing systems at different levels: (1) partial melting of the hydrous upper mantle (Kushiro, 1969; Green, 1973; Wood and Turner, 2009); (2) partial melting of the crust (Beard and Lofgren, 1991); (3) crystal fractionation of hydrous basaltic parental magma (Grove and Baker, 1984; Grove and Kinzler, 1986; Sisson and Grove, 1993; Grove et al., 2003); (4) magma mixing between products of different magma fractionation steps (e.g. basalts and dacites) (Sakuyama, 1981) accompanied by magma mingling (Reubi and Blundy, 2009); (5) mixing between basaltic- and crustal-derived melts (Eichelberger, 1978; Tatsumi and Kogiso, 2003); (6) melting of a subducted slab (Taylor et al., 1969; Defant and Drummond, 1990); and (7) assimilation of the arc crust's plutonic roots (Reubi and Blundy, 2009).

Andesitic Bezymianny Volcano was renowned throughout the world for its catastrophic eruption that occurred on 30 March 1956 after almost 1000 years of dormancy. The mode of explosion was a directed-blast or "Bezymianny-type" eruption (Gorshkov, 1959; Gorshkov and Bogoyavlenskaya, 1965), and volcanological aspects of Bezymianny 
magmatism have been the primary focus in the subsequent 60 years of monitoring the hazard posed by this volcano (e.g. Gordeev, 2006).

Previous petrological and geochemical investigations of Bezymianny Volcano have been mainly focused on volcanic products of historical eruptions, especially those occurring in 1956 and later (Braitseva et al., 1991; Ozerov et al., 1997; Pineau et al., 1999; Tolstykh et al., 1999; Almeev et al., 2002; Dosseto et al., 2003; Turner et al., 2007; Plechov et al., 2008; Shcherbakov et al., 2011). Only a few studies have been devoted to obtaining information on the origin of pre-historic magmas, represented for example by abundant lava domes on the southern slope of Bezymianny edifice (Ermakov, 1977; Braitseva et al., 1991). Therefore, until now, the long-term geochemical history of the Bezymianny plumbing system was not completely understood. In general, identifying the magma source(s) and the contribution of different petrogenetic processes (e.g. crystal fractionation, magma mixing, crustal assimilation) in the evolution of Bezymianny Volcano magmas have been envisioned only for a restricted range of compositions.

Although a basaltic parental magma for Bezymianny andesites is widely accepted in the literature (Maksimov et al., 1978; Kadik et al., 1986; Bogoyavlenskaya et al., 1991), its composition and storage conditions remain unclear. It has been suggested that a magma similar to high-alumina basalt $(\mathrm{HAB})$ derived from a primitive high-magnesia basalt (HMB) of Kliuchevskoi Volcano (these volcanoes are located on opposite slopes of extinct Kamen Volcano, Fig. 1) may represent the parental magma composition of Bezymianny Volcano (Ozerov et al., 1997). Ozerov et al. (1997) compared the results of seismic observations of the deep structures of the two magma plumbing systems, the temporal relationship of the eruption sequences, and the bulk-rock chemistry with the different style of eruptions of these two neighboring volcanoes, and assumed a genetic link between Kliuchevskoi basalts and Bezymianny andesites. They hypothesized that (1) both magma plumbing systems share the same mantle source which generates high-magnesia parental basalt magma (Fig. 2 in Ozerov et al., 1997),
(2) a shallow magma chamber beneath Bezymianny was connected to the Kliuchevskoi magma conduit, whereas the shallow Kliuchevskoi conduit was connected to its eruption vent without a shallow magma chamber, and (3) the geochemical diversity of the magmas from the two volcanoes was attributable to the different crystallization regimes of a common primary magma.

According to Ozerov et al. (1997), the first crystallization regime occurs in the Kliuchevskoi conduit where a hydrous high-magnesia parental magma evolves and produces HAB andesite magma as a result of decompressional crystallization during ascent to the surface. This model is supported by a phase equilibria thermodynamic simulation that assumes (1) a 36\% fractionation of olivine-pyroxene assemblages, (2) from a hydrous $\left(\sim 2\right.$ wt.\% $\left.\mathrm{H}_{2} \mathrm{O}\right)$ mantle-derived parental $\mathrm{HMB}$ in (3) the pressure range from 19 to $7 \mathrm{kbar}$ (decompression crystallization after Ariskin et al., 1995; Ariskin, 1999). According to the calculations, at $7 \mathrm{kbar}, 1110{ }^{\circ} \mathrm{C}$, and about $3 \mathrm{wt} . \% \mathrm{H}_{2} \mathrm{O}$ in the system, plagioclase occurs on the liquidus and the resultant melt is similar to the Kliuchevskoi Volcano HABs.

The second crystallization regime is assumed to proceed in the magma chamber at nearly isobaric conditions and is related to the generation of Bezymianny andesites and dacites. Ozerov et al. (1997) assumes that at a depth of 30-40 km, the Kliuchevskoi magma conduit branches out and connects with the Bezymianny magma chamber located at a depth of between 10 and $20 \mathrm{~km}$. Thus, the Bezymianny chamber is fed by the derivatives of HMBs: HAB andesite melts. These magmas crystallize under essentially isobaric, high-water-activity conditions. Magma evolution in the shallow magma chamber finally generates andesitic to dacitic magmas (Ozerov et al., 1997).

The proposed scenario of the andesite magma genesis along the lineage of HMB-HAB-andesite has been advocated for many island arc volcanoes (Gill, 1981; Brophy, 1986, 1987, 1989; Nye and Reid, 1986; Baker et al., 1994; Heath et al., 1998; Gertisser and Keller, 2000; Smith et al., 2004; Conte et al., 2010) and has been supported by a number of

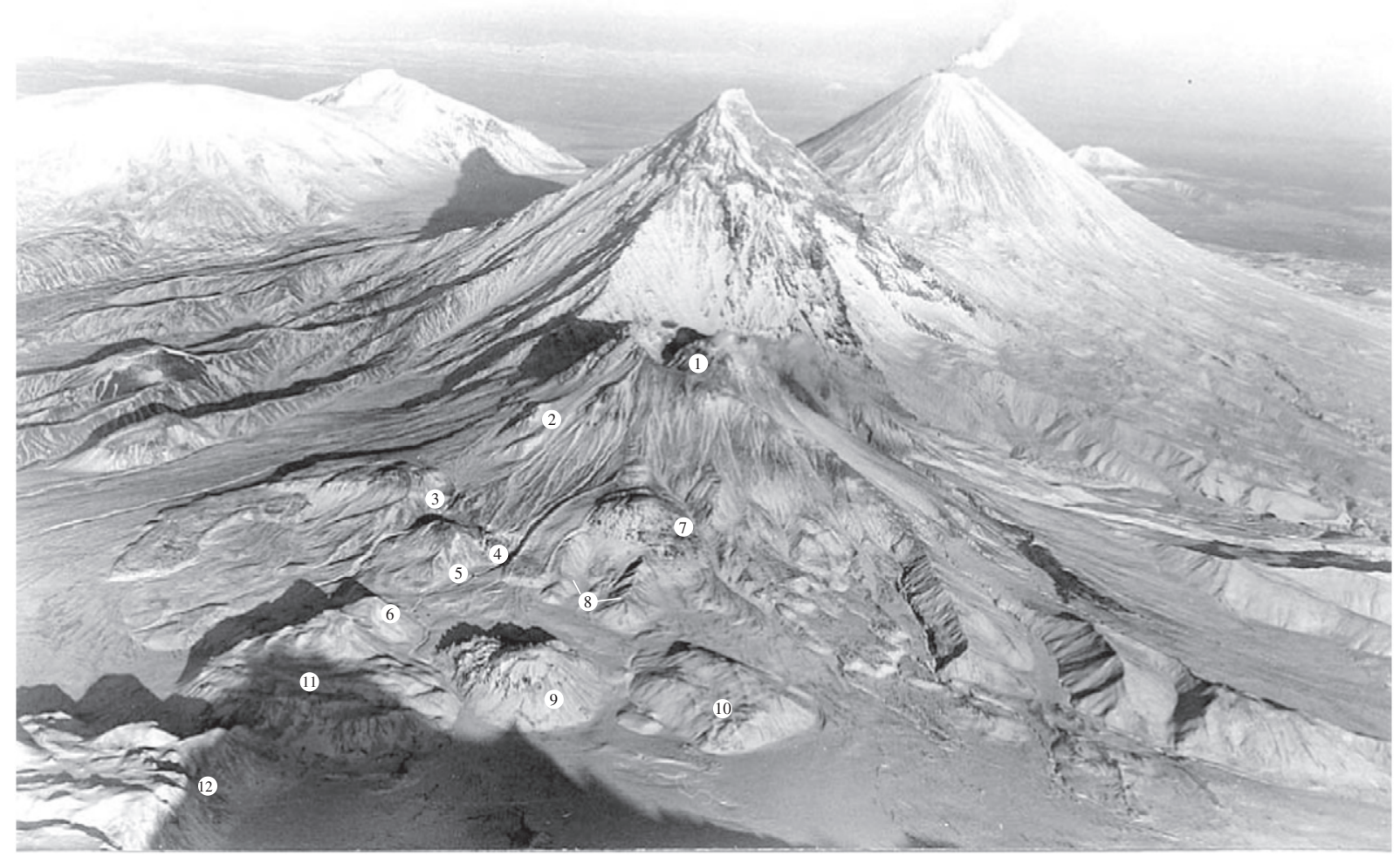

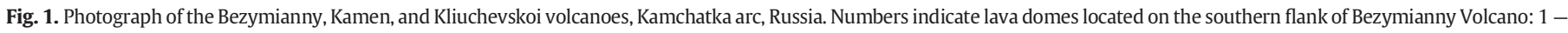

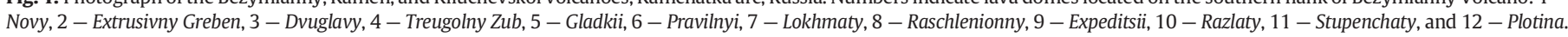
Photograph by Nikolay P. Smelov (05.01.1987). 
experimental studies (Sisson and Grove, 1993; Kawamoto, 1996; Moore and Carmichael, 1998; Martel et al., 1999; Blatter and Carmichael, 2001; Pichavant et al., 2002; Pichavant and Macdonald, 2007). In the case of Bezymianny Volcano, the model proposed by Ozerov et al. (1997) is rather qualitative. Limited geochemical data supported their conclusion about the genetic link between basalts and andesites. The petrochemical trends defined by the Bezymianny lavas were not supported by reliable phase equilibria constraints, e.g. produced in experiments and numeric simulations. Therefore the trends might also be interpreted to result from magma mixing between basaltic and dacitic end-members. In addition, the presence of a large compositional gap existing in Bezymianny between $\mathrm{HAB}$ and basaltic andesite to andesite (between 54 and 57 wt.\% $\mathrm{SiO}_{2}$ ) was not clearly explained (Ozerov et al., 1997), leaving the question about Bezymianny parental composition unresolved.

In this study we present new mineralogical and bulk-rock geochemical data for the intermediate Bezymianny Volcano lavas which span a timescale of magma evolution from $\sim 20 \mathrm{ky}$ to the present. For the first time, we report and discuss variations of some key trace elements in clinopyroxene phenocrysts of the KliuchevskoiBezymianny calc-alkaline volcanic suite which help us to strengthen conclusions about bulk-rock geochemistry. Based on the geochemical data and results of geothermobarometric calculations, we will discuss the genetic relationship between Bezymianny and Kliuchevskoi volcano lavas, the link between "Bezymianny" and "pre-Bezymianny" stage lavas, and the relative role of magma mixing and crystal fractionation, including the effects of pressure and water content, in the genesis of the Bezymianny andesites and dacites.

\section{Geological background and previous work}

\subsection{Regional setting}

Bezymianny Volcano (Fig. 1) belongs to the Kliuchevskoi Group located in the central part of the Central Kamchatka Depression (CKD), a rift-like tectonic structure bounded by the Sredinny Range (SR) to the west and the Eastern Volcanic Front (EVF) to the east (Fig. 2a). These three volcanic zones (corresponding to SR, CKD, and EVF) of the Quaternary volcanism in Kamchatka are developing in a NE-SW direction nearly parallel to the Kuril-Kamchatka trench, above the depth range of the Wadati-Benioff zone 100-140 (EVF), 100-200 (CKD) and $400 \mathrm{~km}$ (SR) (Gorbatov et al., 1997).

The pronounced geochemical variations of the lavas from frontal arc (EVF) to back arc (SR) have been mainly attributed to the decrease in degree of partial melting and (to a lesser extent) by mantle source variability (Churikova et al., 2001; Münker et al., 2004). The along-arc geochemical variations (mainly CKD lavas) are associated with the adjacent Kamchatka-Aleutian arc triple junction where there is a transition of the plate boundary from convergence of the Pacific Plate to oblique strike-slip transform of the western Aleutians (Baranov et al., 1991; Geist and Scholl, 1994; Gorbatov et al., 1997). This exceptional tectonic environment exposed the transform edge of the subducting Pacific Plate slab to the mantle. As a result, this setting manifests the strong geochemical diversity of the CKD magmas (Yogodzinski et al., 2001; Levin et al., 2002; Portnyagin et al., 2005, 2007b; Portnyagin and Manea, 2008) from predominant island-arc tholeiitic in the south (e.g. Tolbachik) to calc-alkaline magmas and high-magnesia andesites to the north (Shiveluch Volcano and the Shisheisky Complex). In addition, the upwelling of hot mantle flow around the edge of the subducting Pacific Plate is believed to be responsible for the extraordinary productivity of the CKD volcanoes (Levin et al., 2002; Portnyagin et al., 2005, 2007b).

\subsection{Plumbing system(s) of Bezymianny and Kliuchevskoi volcanoes from geophysical studies}

The crust below the CKD is about 30 to $40 \mathrm{~km}$ thick and consists of Cretaceous to Tertiary accreted arc and oceanic crust overlain by Pleistocene plateau basalts (Ivanov, 1990; Fedotov et al., 1991). Earlier geophysical studies revealed the existence of "seismic shadow" zones interpreted as a magmatic channel 20 to $60 \mathrm{~km}$ beneath Kliuchevskoi
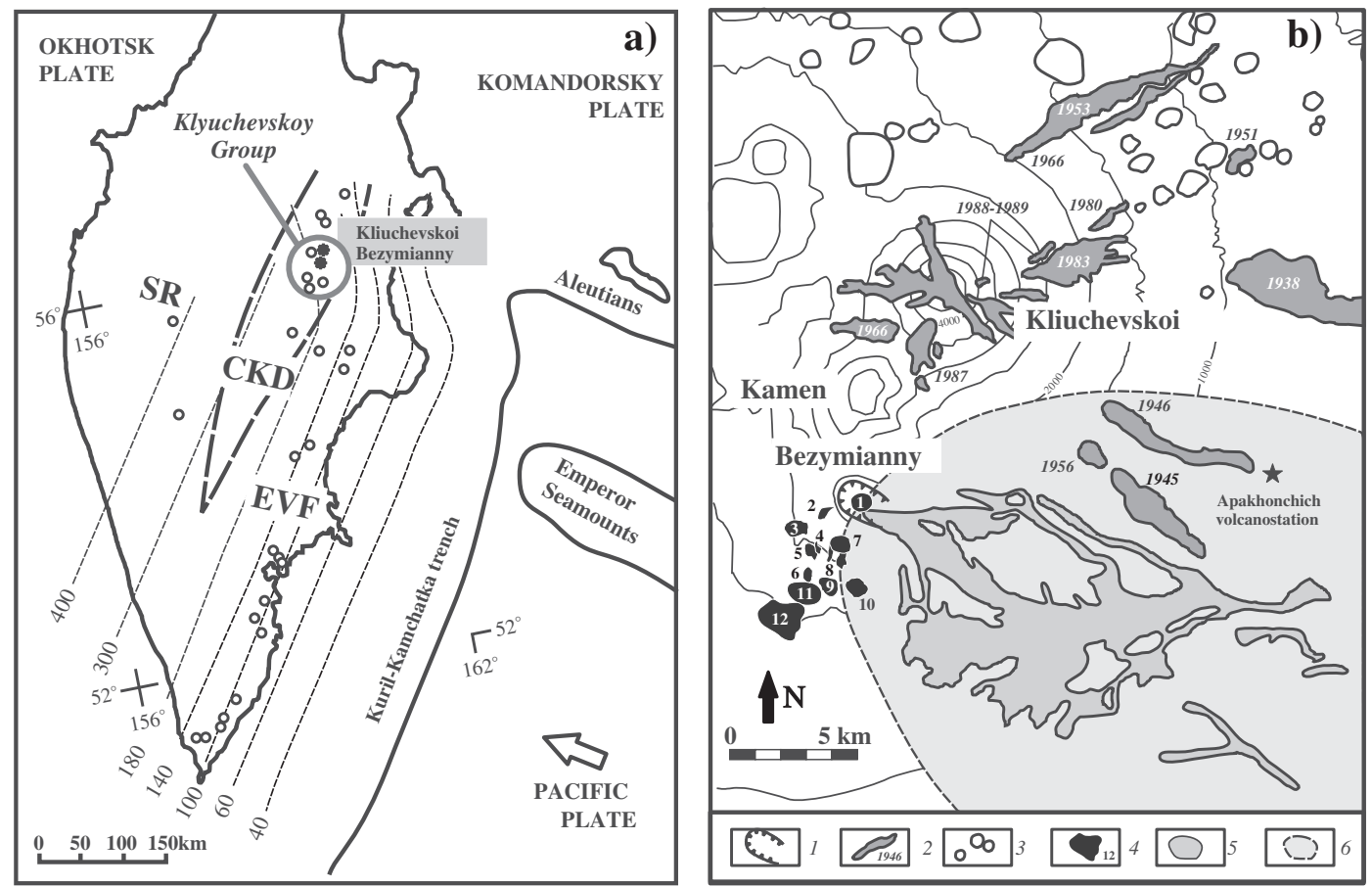

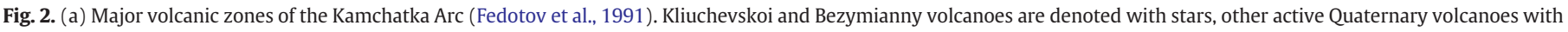

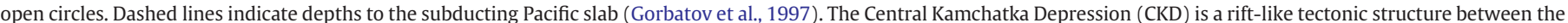

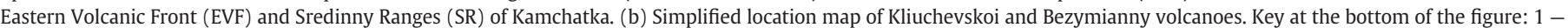

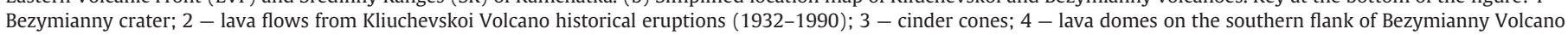

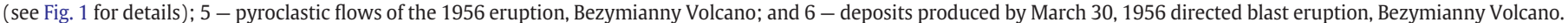


and a crustal magmatic chamber 10-20 km beneath Bezymianny (see review in Ozerov et al., 1997). More recent studies on the seismic tomography revisited the velocity structure of the crust beneath the Kliuchevskoi Group and found large and small low-velocity anomalies beneath Kliuchevskoi at about 25-40 and 5-10 km depth, respectively. The large-velocity anomaly appears narrow at $20 \mathrm{~km}$ depth and broadens laterally at around 30-35 km depth, suggesting a significant amount of magma ponding at the Moho discontinuity level (Lees et al., 2007). The small low-velocity anomaly was related to a number of dikes and small magma channels that exist in the upper part of the volcano plumbing systems (Khubunaya et al., 2007).

The most recent seismic model (Koulakov et al., 2011) suggests the existence of three magma storage levels beneath Kliuchevskoi: (1) in the uppermost mantle to lowermost crust (below $25 \mathrm{~km}$ ), (2) in the upper crust (8-13 km), and (3) at a very shallow depth. Importantly, this study confirmed previous results which indicated the lack of a large magma chamber beneath Kliuchevskoi at an intermediate crustal depth between 13 and $25 \mathrm{~km}$ (Fedotov et al., 1988). Beneath Bezymianny Thelen et al. (2010) identified (1) a shallow magma accumulation zone at 1-1.5 km, and (2) a deeper earthquake-free zone, a magma chamber proxy at $\sim 7 \mathrm{~km}$ depth, directed towards the KamenKliuchevskoi volcanoes. They also argued that the magma plumbing system beneath Bezymianny may be controlled by a large crustal fault which is sub-parallel to the direction of the Bezymianny and Kliuchevskoi vents. Thus, new seismological observations agree with the previous suggestions (Utnasin et al., 1976; Anosov et al., 1978) that a crustal reservoir exists beneath Bezymianny which might be connected to the deep feeding system of Kliuchevskoi Volcano.

\subsection{Eruption history, volcanic activity, and volcanic products}

The geology and eruptive activity of Bezymianny and Kliuchevskoi volcanoes have been thoroughly described in previous studies (Gorshkov and Bogoyavlenskaya, 1965; Ermakov, 1977; Bogoyavlenskaya et al., 1985, 1991; Braitseva et al., 1991; Khrenov et al., 1991; Khubunaya et al., 1993; Kersting and Arculus, 1994; Ariskin et al., 1995; Belousov, 1996; Ozerov et al., 1997; Ozerov, 2000; Belousov et al., 2002). We briefly review these reports below.

\subsubsection{Bezymianny Volcano $\left(55^{\circ} 97^{\prime} \mathrm{N}, 160^{\circ} 58^{\prime} \mathrm{E}\right)$}

Bezymianny is a typical stratovolcano located on the southeastern flank of the extinct Kamen Volcano (Fig. 1). The latest eruption of Bezymianny started on 30 March 1956 . An edifice failure was followed by a gigantic directed blast and subsequent climactic Plinian eruptions. At present the center of the eruption crater is occupied by a Novy lava dome (Fig. 1). Numerous lava flows are radially distributed around the cone. Their length rarely exceeds a few kilometers. An extremely wide area $\left(\sim 500 \mathrm{~km}^{2}\right.$, Belousov, 1996) on the eastern flank of the volcano is covered by the deposits of the directed blast eruption including pyroclastic surges and flows from the 1956, 1984, and 1997 explosive eruptions (Braitseva et al., 1991; Belousov, 1996; Belousov et al., 2002). More voluminous volcanic material is represented by the deposits of two older pyroclastic flows, the Western Pyroclastic Flow and the Eastern Pyroclastic Flow ca. 1000 and 1200 years ago, respectively, covering an area of $25-30 \mathrm{~km}^{2}$ (Braitseva et al., 1991). Twelve large lava domes are situated on the southern-southwestern Bezymianny Volcano slopes (Figs. 1 and 2b). Based on age estimations (Braitseva et al., 1991), they were separated into two groups. The first group includes domes that were formed in Late Pleistocene, prior to the formation of the main Bezymianny stratocone (Braitseva et al., 1991). They form the so-called Late Pleistocene lava-dome complex after Ermakov (1977) and include the dacitic Gladkii, Pravilny, and Raschlenionny domes and more younger (>10-11 kyr, Braitseva et al., 1991) the andesitic Plotina, Stupenchaty, Dvuglavy, Razlaty, and Kulich domes, with features of subglacial extrusion. The second group of Holocene lava domes includes andesitic Expeditsii and Lokhmaty and dacitic Extrusivny Greben and Treugolny Zub domes. The Holocene lava domes grew simultaneously with the formation of the Bezymianny stratocone (4700 14C BP, Braitseva et al., 1995). The detailed stratigraphy of the eruptive products is summarized in Braitseva et al. (1991). According to previous researchers, the compositional range of the Bezymianny lavas and pyroclastic flows varies from 51 to $64 \mathrm{wt} . \% \mathrm{SiO}_{2}$ (Bogoyavlenskaya et al., 1991; Braitseva et al., 1991) with the strong predominance of intermediate andesite.

\subsubsection{Kliuchevskoi Volcano $\left(56^{\circ} 07^{\prime} N, 160^{\circ} 08^{\prime}\right.$ E)}

Kliuchevskoi is a typical stratovolcano built up by lava flows and pyroclastic material and features about 80 parasitic vents (Fig. 2b) represented by cinder cones and extensive lava flows. The volcano is $5800-6000{ }^{14} \mathrm{C}$ BP old (Braitseva et al., 1995). The modern summit eruptions of the volcano are smaller in intensity, and eruption duration ranges from a few weeks up to a few years. Eruption products of the volcano are represented by aa-type lava flows and pyroclastic materials. Volcanic lavas are represented by a spectrum of basalts ranging in composition from HMBs to HABs (Khubunaya et al., 1993; Kersting and Arculus, 1994; Ariskin et al., 1995; Ozerov, 2000; Mironov et al., 2001). However, this traditional and widely-accepted classification is simplified and does not completely represent all compositional peculiarities of Kliuchevskoi rocks: most basalts are rich in $\mathrm{SiO}_{2}$ and located in the basaltic andesite field of the total alkali versus silica (TAS) diagram (see below); some HMBs may have high $\mathrm{Al}$, while some HABs may be high in magnesia (Portnyagin et al., 2007b; Mironov and Portnyagin, 2011). In this study we follow the traditional classification.

\section{Samples}

Fifty nine rock samples were obtained in the course of field campaigns in 1999 and 2001 on the slopes of Bezymianny Volcano and adjacent lava domes. Six samples were provided by Alexander P. Maksimov, including basaltic andesite M-1560 which represents a Kamen Volcano lava flow. For the sake of systematic comparison to previous studies, we follow the eruption age and nomenclatures proposed by Bogoyavlenskaya et al. (1991) and Braitseva et al. (1991). Our entire sample set covers almost all periods of Bezymianny activity (see Supplementary Material 1), including 24 samples from the "preBezymianny" stage when the Late Pleistocene lava-dome complex formed and the "Bezymianny stage" with eruptive phases $B$ ( 8 samples), $B-I$ (10 samples), and B-II ( 7 samples), and the most recent stage $B$-III (10 samples) (see stages in Braitseva et al., 1991). These samples represent all the petrological/chemical varieties of the erupted products. They range from basaltic andesite ( 3 samples), through two-pyroxene andesite (9), orthopyroxene-bearing andesite (14), and hornblende-bearing andesite (18), to dacite (16).

Samples of HMB (4), magnesian basalt (3), aluminous basalt (4), and HAB (4) from Kliuchevskoi were also selected from the collection of Ariskin et al. (1995). These 15 samples had been previously analyzed for major and some trace elements at the Vernadsky Institute (Moscow) in 1992-1993. To avoid possible inter-laboratory biases, the chemical analyses of these samples have been fully replicated in the course of this study.

Volcanic lavas were analyzed for major, trace, and isotopic ( $\mathrm{Sr}, \mathrm{Nd}$, $\mathrm{Pb}$ ) compositions (Tables 1-3). Major and trace elements were also analyzed in olivine $(\mathrm{Ol})$, clinopyroxene $(\mathrm{Cpx})$, orthopyroxene $(\mathrm{Opx})$, plagioclase (Plag), hornblende ( $H b l)$, magnetite $(M t)$, and ilmenite ( Ilm) from representative lavas. The details of analytical techniques are provided in Supplementary Material 2. Bezymianny mineral compositions are provided in Supplementary Material 3. Kliuchevskoi mineral compositions are from Ariskin et al. (1995) and Ozerov (2000). Modal proportions of phenocrysts and liquid compositions calculated by point-counting are presented in Table 1 (in volume percent on a vesicle-free basis). The average major element compositions of the 
Table 1

Modal proportions (vol.\%) of phenocrysts in the Kliuchevskoi and Bezymianny lavas.

\begin{tabular}{|c|c|c|c|c|c|c|c|c|c|c|c|c|c|c|c|c|}
\hline \multirow{3}{*}{$\begin{array}{l}\text { Group } \\
\text { Rock type } \\
\text { Sample }\end{array}$} & \multirow{2}{*}{\multicolumn{2}{|c|}{ KL-I }} & \multirow{3}{*}{$\frac{\frac{\text { KL-II }}{\text { Mg-basalt }}}{\text { KL-45 }}$} & \multirow{2}{*}{\multicolumn{2}{|c|}{$\frac{\text { KL-III }}{\text { Al basalt }}$}} & \multirow{2}{*}{\multicolumn{2}{|c|}{$\begin{array}{l}\text { KL-IV } \\
\mathrm{HAB}\end{array}$}} & \multirow{2}{*}{\multicolumn{2}{|c|}{$\frac{\text { BZ-I }}{\text { Basaltic andesite }}$}} & \multirow{2}{*}{\multicolumn{2}{|c|}{$\frac{\text { BZ-II }}{2 P x \text { andesite }}$}} & \multirow{3}{*}{$\frac{\frac{\text { BZ-III }}{\text { Opx andesite }}}{\text { OB-42 }}$} & \multirow{2}{*}{\multicolumn{2}{|c|}{$\frac{\mathrm{BZ}-\mathrm{IV}}{\mathrm{Hbl} \text { andesite }}$}} & \multirow{2}{*}{\multicolumn{2}{|c|}{$\frac{\text { BZ-V }}{\text { Dacite }}$}} \\
\hline & & & & & & & & & & & & & & & & \\
\hline & KL-3 & KL-12 & & KL-35 & KL-40 & KL-1 & KL-31 & OB-21 & OB-19 & OB-9 & OB-11 & & M-1412 & OB-16 & OB-15 & OB-40 \\
\hline $\mathrm{SiO}_{2}$ (wt.\%) & 51.82 & 52.35 & 53.13 & 53.26 & 53.74 & 54.85 & 54.1 & 53.87 & 53.17 & 56.32 & 55.85 & 57.5 & 60.54 & 63.87 & 66.78 & 65.64 \\
\hline MgO (wt.\%) & 12.10 & 11.00 & 8.98 & 6.50 & 7.83 & 5.20 & 4.81 & 5.23 & 5.81 & 5.38 & 4.97 & 3.43 & 2.73 & 2.23 & 1.22 & 1.1 \\
\hline Points & 21,000 & 14,600 & 15,300 & 15,000 & 8900 & 8000 & 4000 & 7000 & 8000 & 5000 & 6000 & 5000 & 4000 & 4000 & 4000 & 6000 \\
\hline \multicolumn{17}{|c|}{ Vesicle-free amounts of phenocrysts and groundmass (vol.\%) } \\
\hline $\mathrm{Ol}$ & 8.5 & 8.1 & 3.6 & 2.7 & 3.6 & 1.0 & 0.3 & 0.5 & 2.2 & 0.2 & 2.5 & - & - & - & - & - \\
\hline$C p x$ & 9.1 & 11.0 & 7.7 & 5.3 & 8.8 & 0.3 & 1.1 & 4.8 & 2.1 & 6.6 & 2.5 & - & - & - & - & - \\
\hline Opx & 0.1 & - & - & - & - & - & - & 1.5 & 0.5 & 2.5 & 0.3 & 3.5 & 1.7 & - & - & - \\
\hline Oxide $(S p, M t)$ & 0.1 & - & - & 0.5 & 0.4 & 0.3 & - & 1.0 & 0.4 & 0.4 & 0.1 & 0.4 & 0.6 & 0.9 & 0.3 & 0.4 \\
\hline Plag & - & 1.0 & - & 13.5 & 6.4 & 10.9 & 3.1 & 13.7 & 13.9 & 16.5 & 0.6 & 3.3 & 18.3 & 13.7 & 9.8 & 4.0 \\
\hline $\mathrm{Hbl}$ & - & - & - & - & - & - & - & 2.5 & - & - & - & 1.4 & 3.1 & 11.2 & 5.0 & 1.8 \\
\hline Groundmass & 82.2 & 79.9 & 88.8 & 77.9 & 80.8 & 87.5 & 95.5 & 76.0 & 80.8 & 73.8 & 94.0 & 91.4 & 76.3 & 74.3 & 85.0 & 93.8 \\
\hline Vesicles & 19.6 & 4.3 & 1.9 & 2.3 & 0.8 & 0.2 & 33.4 & 0.6 & - & 0.3 & 0.3 & 3.6 & 5.7 & 7.2 & - & - \\
\hline \multicolumn{17}{|c|}{ Calculated groundmass (liquid) compositions (wt.\%) } \\
\hline $\mathrm{SiO}_{2}$ & 53.06 & 53.59 & 53.62 & 55.01 & 55.26 & 55.95 & 54.30 & 56.43 & 55.01 & 57.42 & 56.48 & 58.36 & 63.41 & 70.31 & 69.89 & 67.11 \\
\hline $\mathrm{TiO}^{2}$ & 0.96 & 1.02 & 0.99 & 1.17 & 1.04 & 1.20 & 1.18 & 1.12 & 1.27 & 1.13 & 1.08 & 0.78 & 0.60 & 0.15 & 0.31 & 0.35 \\
\hline $\mathrm{Al}_{2} \mathrm{O}_{3}$ & 15.87 & 16.37 & 15.78 & 16.14 & 16.32 & 16.17 & 17.63 & 17.22 & 16.33 & 16.02 & 17.87 & 18.73 & 16.37 & 15.69 & 16.20 & 17.30 \\
\hline $\mathrm{FeO}_{\text {tot }}$ & 8.39 & 8.81 & 8.82 & 8.88 & 8.45 & 9.03 & 8.72 & 8.81 & 9.60 & 9.07 & 7.62 & 6.76 & 5.99 & 2.92 & 3.04 & 3.65 \\
\hline $\mathrm{MnO}$ & 0.15 & 0.15 & 0.15 & 0.16 & 0.16 & 0.17 & 0.15 & 0.17 & 0.19 & 0.10 & 0.15 & 0.12 & 0.13 & 0.12 & 0.11 & 0.14 \\
\hline MgO & 7.95 & 6.69 & 7.12 & 5.97 & 5.88 & 4.98 & 5.11 & 4.51 & 5.28 & 4.63 & 3.68 & 2.64 & 2.46 & 0.95 & 0.70 & 0.92 \\
\hline $\mathrm{CaO}$ & 10.18 & 9.61 & 9.44 & 7.91 & 8.24 & 7.18 & 7.77 & 6.89 & 7.48 & 6.71 & 8.17 & 7.35 & 5.83 & 3.54 & 3.49 & 4.29 \\
\hline $\mathrm{Na}_{2} \mathrm{O}$ & 2.61 & 2.79 & 2.84 & 3.58 & 3.20 & 3.57 & 3.56 & 3.45 & 3.46 & 3.13 & 3.27 & 3.74 & 3.30 & 3.86 & 3.85 & 4.46 \\
\hline $\mathrm{K}_{2} \mathrm{O}$ & 0.70 & 0.81 & 1.07 & 0.95 & 1.25 & 1.49 & 1.34 & 1.17 & 1.16 & 1.55 & 1.48 & 1.29 & 1.69 & 2.23 & 2.22 & 1.51 \\
\hline $\mathrm{P}_{2} \mathrm{O}_{5}$ & 0.15 & 0.16 & 0.18 & 0.23 & 0.21 & 0.25 & 0.23 & 0.23 & 0.21 & 0.24 & 0.21 & 0.25 & 0.23 & 0.23 & 0.20 & 0.27 \\
\hline
\end{tabular}

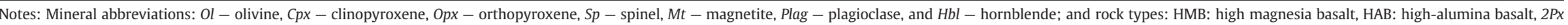
andesite: two-pyroxene bearing andesite, $O p x$ andesite: orthopyroxene bearing andesite, and $\mathrm{Hbl}$ andesite: hornblende bearing andesite. 
Table 2

Mean compositions and normative mineralogy of the main Kliuchevskoi and Bezymianny volcano petrochemical groups.

\begin{tabular}{|c|c|c|c|c|c|c|c|c|c|c|}
\hline Group & KL-I & KL-II & KL-III & KL-IV & $\begin{array}{l}\text { M-1560 } \\
\text { (Kamen volc.) }\end{array}$ & BZ-I & BZ-II & BZ-III & BZ-IV & BZ-V \\
\hline Rock type & HMB & Mg-basalt & Al basalt & $\mathrm{HAB}$ & Basalt & $\begin{array}{l}\text { Basaltic } \\
\text { andesite }\end{array}$ & $2 P x$ andesite & Opx andesite & $\mathrm{Hbl}$ andesite & Dacite \\
\hline Number of samples [wt.\%] & 15 & 46 & 50 & 131 & 1 & 3 & 9 & 14 & 18 & 16 \\
\hline $\mathrm{SiO}_{2}$ & $51.76(0.34)$ & $53.39(0.55)$ & $53.22(0.78)$ & $53.5(0.48)$ & 52.84 & $53.26(0.41)$ & $56.27(0.24)$ & $57.52(0.52)$ & $61.21(1.64)$ & $66.21(1.25)$ \\
\hline $\mathrm{TiO}_{2}$ & $0.86(0.07)$ & $0.84(0.11)$ & $0.95(0.13)$ & $1.09(0.07)$ & 0.94 & $1.1(0.05)$ & $0.88(0.06)$ & $0.8(0.05)$ & $0.62(0.06)$ & $0.4(0.03)$ \\
\hline $\mathrm{Al}_{2} \mathrm{O}_{3}$ & $13.86(0.33)$ & $15.29(0.55)$ & $16.79(0.66)$ & $18.26(0.5)$ & 17.05 & $17.75(0.44)$ & $16.59(0.25)$ & $18(0.29)$ & $17.32(0.65)$ & $17.48(0.39)$ \\
\hline $\mathrm{FeO}_{\text {tot }}$ & $8.83(0.18)$ & $8.52(0.24)$ & $8.83(0.47)$ & $8.67(0.33)$ & 8.68 & $9.22(0.16)$ & $7.97(0.1)$ & $7.56(0.34)$ & $6.07(0.58)$ & $3.96(0.44)$ \\
\hline $\mathrm{MnO}$ & $0.17(0.01)$ & $0.17(0.02)$ & $0.17(0.04)$ & $0.16(0.02)$ & 0.15 & $0.17(0)$ & $0.16(0)$ & $0.15(0.01)$ & $0.14(0.01)$ & $0.13(0.01)$ \\
\hline $\mathrm{MgO}$ & $11.55(0.43)$ & $8.58(0.65)$ & $6.89(1)$ & $5.24(0.34)$ & 7.05 & $5.39(0.37)$ & $5.32(0.23)$ & $3.72(0.3)$ & $2.98(0.73)$ & $1.12(0.16)$ \\
\hline $\mathrm{CaO}$ & $9.73(0.2)$ & $9.41(0.3)$ & $8.91(0.46)$ & $8.22(0.38)$ & 9.35 & $8.82(0.16)$ & $8.42(0.14)$ & $7.45(0.17)$ & $6.49(0.54)$ & $4.67(0.35)$ \\
\hline $\mathrm{Na}_{2} \mathrm{O}$ & $2.47(0.18)$ & $2.72(0.24)$ & $3.11(0.32)$ & $3.45(0.2)$ & 3.05 & $3.07(0.07)$ & $3.01(0.06)$ & $3.4(0.14)$ & $3.52(0.15)$ & $4.12(0.19)$ \\
\hline $\mathrm{K}_{2} \mathrm{O}$ & $0.63(0.09)$ & $0.9(0.15)$ & $0.96(0.19)$ & $1.2(0.1)$ & 0.74 & $0.91(0.03)$ & $1.2(0.08)$ & $1.2(0.09)$ & $1.45(0.18)$ & $1.7(0.24)$ \\
\hline $\mathrm{P}_{2} \mathrm{O}_{5}$ & $0.15(0.02)$ & $0.18(0.03)$ & $0.18(0.04)$ & $0.2(0.04)$ & 0.15 & $0.17(0)$ & $0.18(0.01)$ & $0.2(0.03)$ & $0.19(0.03)$ & $0.21(0.04)$ \\
\hline $\mathrm{Mg} /(\mathrm{Mg}+\mathrm{Fe})$ & 0.69 & 0.64 & 0.58 & 0.52 & 0.59 & 0.51 & 0.54 & 0.47 & 0.47 & 0.34 \\
\hline $\mathrm{CaO} /\left(\mathrm{CaO}+\mathrm{Al}_{2} \mathrm{O}_{3}\right)$ & 0.56 & 0.53 & 0.49 & 0.45 & 0.50 & 0.47 & 0.48 & 0.43 & 0.41 & 0.33 \\
\hline$\Sigma$ alkalis & 3.10 & 3.62 & 4.07 & 4.65 & 3.79 & 3.98 & 4.21 & 4.6 & 4.97 & 5.82 \\
\hline \multicolumn{11}{|l|}{ CIPW, norm } \\
\hline $\mathrm{Qtz}$ & - & - & - & - & - & 0.72 & 5.24 & 7.51 & 13.46 & 21.53 \\
\hline An & 24.87 & 26.85 & 29.02 & 30.79 & 30.65 & 31.97 & 28.21 & 30.31 & 27.18 & 21.80 \\
\hline$A b$ & 20.90 & 23.02 & 26.32 & 29.19 & 25.81 & 26.06 & 25.47 & 28.77 & 29.79 & 34.86 \\
\hline Or & 3.72 & 5.32 & 5.67 & 7.09 & 4.37 & 5.38 & 7.09 & 7.09 & 8.57 & 10.05 \\
\hline Cor & - & - & - & - & - & - & - & - & - & 0.87 \\
\hline$D i$ & 18.18 & 15.24 & 11.54 & 7.21 & 12.14 & 8.95 & 10.25 & 4.47 & 3.16 & - \\
\hline Hy & 17.90 & 25.05 & 21.93 & 21.20 & 21.20 & 24.42 & 21.65 & 19.87 & 16.22 & 9.64 \\
\hline $\mathrm{Ol}$ & 12.45 & 2.51 & 3.31 & 1.98 & 3.70 & - & - & - & - & - \\
\hline $\mathrm{Ilm}$ & 1.63 & 1.60 & 1.80 & 2.07 & 1.79 & 2.09 & 1.67 & 1.52 & 1.18 & 0.76 \\
\hline$A p$ & 0.35 & 0.42 & 0.42 & 0.46 & 0.35 & 0.39 & 0.42 & 0.46 & 0.44 & 0.49 \\
\hline
\end{tabular}

main lava types are given in Table 2. The entire dataset of major, trace, and isotope compositions is summarized in Table 3.

\section{Results}

\subsection{Statistical grouping and normative mineralogy}

Ariskin et al. (1995) applied a conventional cluster analysis to the Kliuchevskoi basalts and distinguished four chemical groups: HMBs (Group KL-I), magnesian basalts (KL-II), aluminous basalts (KL-III), and HABs (KL-IV). A similar statistical approach applied to the Bezymianny samples allowed us to distinguish five statistical groups (Table 2, see also dendrograph plot in Supplementary Material 4).

The separation of five statistical groups correlates well with lava petrography. The entire compositional space can be subdivided on the basis of presence and absence of $\mathrm{Hbl}$ in the lavas. The " $\mathrm{Hbl}$-free" cluster contains two distinctive groups with less-differentiated basaltic andesites (BZ-I) and intermediate andesites. The andesite cluster can be subdivided in two groups: andesites with Cpx (BZ-II) and andesites without $C p x$ (or in which $C p x$ is secondary to $O p x$; group BZ-III). The "Hbl-bearing" group contains two sub-clusters of $\mathrm{Hbl}$ andesites (BZ-IV) and dacites (BZ-V). Although the basaltic andesite from Kamen Volcano (sample M-1560) may be grouped together with Bezymianny basaltic andesites, it exhibits slightly different petrochemical characteristics in $\mathrm{Mg} /(\mathrm{Mg}+\mathrm{Fe}), \mathrm{K}_{2} \mathrm{O}, \mathrm{CaO} /\left(\mathrm{CaO}+\mathrm{Al}_{2} \mathrm{O}_{3}\right)$, total alkalis, and mineralogy. Therefore this sample is considered separately from the BZ-I group.

The average compositions of KL-I to KL-IV and BZ-I to BZ-V were further used to calculate their normative mineralogy (Table 2). All the samples are hypersthene-normative. HMB to HAB from Kliuchevskoi, including basalt from Kamen Volcano (M-1560), are Ol-normative whereas intermediate Bezymianny lavas are quartz (Qtz) normative. The transition from HMB to dacite throughout the whole volcanic suite is accompanied by monotonic decrease in normative $\mathrm{Ol}$ and diopside ( $\mathrm{Di}$ in Table 2). Normative Plag is almost constant, but the proportion of Plag end-member components is changed: albite ( $A b$ in Table 2 ) and orthoclase (Or in Table 2) increase whereas the anorthite (An in
Table 2) component decreases. Collectively, lava compositions from both volcanoes define a silica saturation trend typical of calc-alkaline arc magmas. The Kliuchevskoi-Bezymianny lava series begins with the "Ol-tholeiite" field of the basalt tetrahedron (Yoder and Tilley, 1962), crosses the plane of silica saturation, continues towards the Qtz tholeiite subspace, and finally reaches the side where Di-free or corundum-bearing compositions are located (not shown). This general evolutionary path is consistent with the crystallization of $\mathrm{Plag}+\mathrm{Ol}+\mathrm{Cpx}+\mathrm{Mt}$ (POAM association after Gill (1981).

\subsection{Petrography and mineral chemistry}

\subsubsection{HMB to HAB basaltic suite of Kliuchevskoi Volcano}

Kliuchevskoi lavas (KL-I $=$ HMB to KL-IV $=\mathrm{HAB}$ ) are vesicular or non-vesicular basalts with medium- to coarse-grained, porphyritic to microphyric textures. They contain abundant $\mathrm{Ol}$ and $\mathrm{Cpx}$ phenocrysts and aluminous varieties have additional Plag. Total phenocryst volume varies from 10 to 20 vol.\% (Table 1). Cpx always prevails in a mafic assemblage although the $\mathrm{Ol} / \mathrm{Cpx}$ ratio is not constant, ranging from 0.9 in HMB to 0.3 in HAB. Ol crystals range from 0.1 to $2 \mathrm{~mm}$ in size and their shapes vary, from euhedral to subhedral in HMB to euhedral and anhedral in HAB. In general, normal crystal zoning dominates throughout the entire basaltic series, and compositions cover the interval of $\mathrm{Mg} \#_{90-64}\left(\mathrm{Mg} \#=\mathrm{Mg} /\left(\mathrm{Mg}+\mathrm{Fe}^{*}\right)\right.$, molar, where $\mathrm{Fe}^{*}$ is total Fe expressed as $\mathrm{Fe}^{2+}$ ), including phenocrysts, subphenocrysts, and microlites. $C p x$ crystals are commonly smaller than $2 \mathrm{~mm}$ in diameter, but occasionally large aggregates can reach $3.0-3.5 \mathrm{~mm}$. Large phenocrysts are anhedral, whereas smaller phenocrysts are euhedral. Cpx cores are mainly diopside to $\mathrm{Mg}$-rich augite (Ozerov, 2000). Cpx Mg\# ranges in the interval of $\mathrm{Mg}_{93-65}$. Groundmass microlites have the same composition as microphenocryst rims, but some of them are pigeonites. The subordinate $O p x$ is sporadically observed as a phenocryst $(0.5-0.1 \mathrm{~mm})$ in magnesian basalts and rarely in HAB. Opx was also identified as inclusions in both $\mathrm{Ol}$ and $C p x$ in all rock types. Compositionally $\mathrm{Opx}$ ranges from $\mathrm{Mg} \#_{85}$ to Mg\# ${ }_{65}$. Abundance of Plag gradually and systematically increases from HMB to HAB. In HMB Plag occurs rarely as small euhedral sub- 
to micro-phenocrysts with average composition of $\mathrm{An}_{70}$. In $\mathrm{HAB}$, Plag forms euhedral and tabular or prismatic crystals, up to $2 \mathrm{~mm}$ in length, with normal, oscillatory, and reverse zoning. Plag in aluminous and some magnesian basalts exhibits complex zoning patterns, normal-to-reverse, reverse-to-normal, reverse, and normal in the same sample. Compositions of Plag phenocrysts and microphenocrysts in $\mathrm{HAB}$ vary widely, from $\mathrm{An}_{85}$ in the cores to $\mathrm{An}_{50}$ in the rims, with normal and occasionally complex zoning patterns. Cr-rich spinel (Sp) occurs as inclusions in $\mathrm{Ol}$, especially from $\mathrm{HMB}$ and magnesian basalt. In Plag crystals from an Apakhonchich lava flow HAB, rare amphibole in melt inclusions have been reported (Plechov et al., 2000), although hydrous minerals are generally not present in the Kliuchevskoi basalts.

\subsubsection{Basaltic andesites of Bezymianny Volcano}

Basaltic andesites from Bezymianny (BZ-I) are porphyritic to seriate porphyritic rocks, containing 15-25 vol.\% phenocrysts of Plag ( 14\%), Cpx (2-5\%), Ol (0.5-2\%), Opx (0.5-1.5\%), and Mt (0.5-1\%) (Table 1). The intergranular to intersertal groundmass is composed of Plag, Cpx, $M t$, and glass. Plag ( $\left.\mathrm{An}_{90-60}\right)$ is the dominant phenocryst in basaltic andesites (Fig. 3) as well as in all more evolved rocks, and constitutes about $70 \%$ of the phenocryst volume. It exhibits tabular euhedral to subhedral forms, and ranges from 0.2 to $2 \mathrm{~mm}$ in the longest dimension, with a typical size of $0.5-1 \mathrm{~mm}$. Plag phenocrysts display both oscillatory and normal/reverse zoning patterns; some crystals have sieved textures. $\mathrm{Cpx}\left(\mathrm{Mg \#}_{85-65}\right)$ and $\mathrm{Ol}$ (Forsterite (Fo) 80-60 $\left._{80}\right)$ phenocrysts are euhedral to subhedral and range in size from 0.5 to $1.5 \mathrm{~mm}$. Opx $\left(\mathrm{Mg}_{80-60}\right.$ ) are euhedral crystals $\sim 0.5 \mathrm{~mm}$ in size and are always subordinate to Cpx. Mt occurs only as microphenocrysts and inclusions in minerals. Sample OB-21 differs from other basaltic andesites by the presence of rare cristobalite, and subhedral $\mathrm{Hbl}$ up to $7 \mathrm{~mm}$ in length. These $\mathrm{Hbl}$ crystals are surrounded by opacite rims composed of micro grains of $M t$, pyroxenes (Px), and Plag, showing evidence of having reacted with the host magma. $\mathrm{Hbl}$ from OB-21 is the most magnesian amphibole from Bezymianny (Fig. 3) and compositionally it is very close to the amphiboles from the $\mathrm{Cpx}-\mathrm{Hbl}$-bearing cumulates which are frequently observed in $\mathrm{Hbl}$ andesites from the Plotina lava dome (Almeev, 2005).

\subsubsection{Andesites of Bezymianny Volcano}

Andesites exhibit porphyritic to seriate porphyritic textures; they consist of Plag, Cpx, Opx, $\mathrm{Hbl}$, and $\mathrm{Mt}$ phenocrysts, and groundmass in different proportions. Phenocryst amounts vary from 6 to 25 vol.\% (Table 1). The andesites can be subdivided petrographically into three sub-groups using the predominant mafic mineral. We distinguished two-pyroxene (2Px), Opx-, and $\mathrm{Hbl}$-bearing andesites. $2 P x$ andesites are characterized by the predominance of $C p x$ over $O p x$. In Opx andesites high-Ca $P x$ is rare and is always subordinate to $O p x$. $H b l$ andesites contain almost no $C p x$, and amphibole always prevails over $O p x$. These petrographical varieties are consistent with petrochemical groups BZ-II, BZ-III, and BZ-IV, as noted above.

Plag is the ubiquitous mineral in all three groups. In general, Plag predominates over mafic phenocrystic minerals except for the $2 P x$ andesite OB-11. We observed two populations of Plag phenocrysts. One is represented by small $(<1 \mathrm{~mm})$ euhedral tabular crystals with normal and, less commonly, reverse zoning patterns. The other includes large (1-3 mm) subhedral to anhedral phenocrysts with sieved textures or spongy cores with complex zoning patterns, containing numbers of tiny mineral (apatite, $O p x$ ) and glass inclusions. Plag from andesites varies from $\mathrm{An}_{92}$ to $\mathrm{An}_{40}$ (Fig. 3). Plag in $2 \mathrm{Px}$ andesites is characterized by the presence of two distinctive peaks in An content at $\sim \mathrm{An}_{85-80}$ and $\mathrm{An}_{58-52}$. The majority of Plag crystals from $\mathrm{Opx}$ and $\mathrm{Hbl}$ andesites have a mode at $A n_{58-60}$, although some samples are characterized by a bimodal pattern (e.g., OB-16; Fig. 3).

Mafic minerals in $2 P x$ andesites are represented by euhedral to subhedral phenocrysts of $\mathrm{Cpx}\left(\mathrm{Mg}_{85-65}\right)$ and $\mathrm{Opx}\left(\mathrm{Mg} \#_{80-60}\right)$ and rare subhedral to anhedral $\mathrm{Ol}\left(\mathrm{Fo \#}_{90-70}\right)$ in the most magnesian andesites. The typical $O l, C p x$, and $O p x$ phenocryst size varies from 0.7 to $0.3 \mathrm{~mm}$. The amount of $\mathrm{Ol}$ in the lavas varies inversely with the amount of $O p x$ : more $O l$ means less $O p x$, and vice versa.

In Opx andesites, $O p x\left(\mathrm{Mg \#}_{75-60}\right)$ are euhedral to subhedral, 0.3$0.7 \mathrm{~mm}$ in size. $\mathrm{Cpx}$ is rare with $\mathrm{Mg} \#_{75-65}$. One $\mathrm{Opx}$ andesite (OB-30) contains only Plag phenocrysts $<0.5 \mathrm{~mm}$ in size embedded in a hypoto holocrystalline groundmass composed of Plag, Opx, and Mt.

$\mathrm{Hbl}$ andesites contain $\mathrm{Hbl}$ as the principal mafic mineral, although Opx $\left(\mathrm{Mg}_{80-55}\right)$ is also present in some lavas. Cpx phenocrysts are absent, but xenocrystic C $p x$ in andesite from the Expeditsii lava dome (OB-2) has been found. Most lavas in this group are composed of an $\mathrm{Hbl}$-Plag phenocrystic assemblage. $\mathrm{Hbl}$ occurs as euhedral and unzoned crystals $0.5-4 \mathrm{~mm}$ in size. Compositionally these crystals vary from pargasite to tschermakitic pargasite with an increasing proportion of tschermakitic pargasite in the evolved andesites. $\mathrm{Hbl}$ crystals very often exhibit opacite reactive rims. The degree of opacitization varies as shown by the thickness of opacite margins, Some $\mathrm{Hbls}$ are fully replaced by Plag-Cpx-Mt microgranular pseudomorphs (Plechov et al., 2008). Fe-Ti oxides are present as microphenocrysts ( $<0.2 \mathrm{~mm})$ in all groups of andesites. Apatite is present in some $\mathrm{Hbl}$ andesites as microphenocrysts or abundant inclusions in Plag.

\subsubsection{Dacites of Bezymianny Volcano}

Dacites are porphyritic and contain phenocrystic Plag (5-10 vol.\%), green to brown, euhedral to subhedral, or strongly resorbed oxidized $\mathrm{Hbl}$ (2-5 vol.\%), and $\mathrm{Mt}$ microphenocrysts ( 0.5 vol.\%) (Table 1$)$. The groundmass (85-93 vol.\%) is composed of Plag and opaque mineral microlites.

Similar to what occurs in andesites, Plag is the most ubiquitous mineral in dacite; it occurs as single and twinned crystals with normal or reverse zoning and a wide compositional range from $\mathrm{An}_{85}$ to $\mathrm{An}_{50}$. The An content is bimodally distributed (Fig. 3). Large subhedral to anhedral Plag phenocrysts always exhibit sieved textures and reach $5 \mathrm{~mm}$ in size. Plag subphenocrysts show a normal zoning pattern with clear euhedral tabular shape and vary from 0.2 to $0.5 \mathrm{~mm}$ in size.

$\mathrm{Hbl}$ from Pravilnyi and Gladkii domes demonstrates opacite rims but is still fresh in cores, particularly in large crystals up to $7 \mathrm{~mm}$ in size. $\mathrm{Hbl}$ from dacites have the most Fe-rich composition $\left(\mathrm{Mg}_{60-50}\right)$. $\mathrm{Hbl}$ from the most evolved Raschlenionny lava dome are completely opacitized.

\subsubsection{Evolution of mineral compositions}

In Fig. 3, histograms of mineral compositions are given for all Bezymianny Volcano lava types and for a basalt sample from Kamen Volcano. They are arranged in order of increasing host rock $\mathrm{SiO}_{2}$ from top to bottom; the left bottom inset highlights the samples for which mineral phase compositions have been investigated (Fig. 3). Oxide mineral compositions are given for both microphenocrysts and inclusions in minerals.

In general, $\mathrm{Ol}, \mathrm{Cpx}, \mathrm{Opx}$, and $\mathrm{Hbl}$ cores display wide compositional ranges and unimodal $\mathrm{Mg \#}$ distribution in basaltic andesite, and more complex patterns and even bimodal $\mathrm{Mg} \#$ distribution in andesites (particularly in 2Px andesites). Plag core compositions span wider ranges and their histograms are often polymodal. Throughout the entire lava sequence from basaltic andesite to dacite, $C p x, O p x$, and $\mathrm{Hbl}$ cores tend to evolve to Fe-rich compositions and display wide varia-

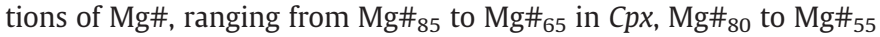
in $\mathrm{Opx}$, and $\mathrm{Mg}_{75}$ to $\mathrm{Mg} \#_{50}$ in $\mathrm{Hbl}$. The $\mathrm{Mg} \#$ modes of these minerals tend to decrease with increasing silica content of the host lava.

$\mathrm{Ol}$ compositions in $2 \mathrm{Px}$ andesites are more magnesian than those from basaltic andesites, although $\mathrm{MgO}$ contents of the host lavas are almost identical. In basaltic andesites, $\mathrm{Ol}$ tends to be more Fe-rich in host lavas with lower MgO content.

Plag compositions vary widely, from $\mathrm{An}_{92}$ in basaltic andesite to $\mathrm{An}_{50-40}$ in $\mathrm{Hbl}$-bearing andesites and dacites. An content in many samples is characterized by a bimodal pattern, with two distinctive peaks at $A n_{85-80}$ and $A n_{65-60}$ in basaltic andesites, $A n_{85-80}$ and $A n_{50}$ 
Table 3

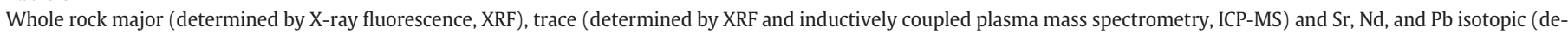

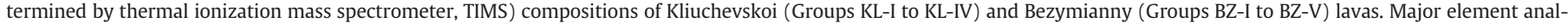
yses are normalized to $100 \%$ on a volatile-free basis with all iron expressed as $\mathrm{FeO}_{\text {total }}$.

\begin{tabular}{|c|c|c|c|c|c|c|c|c|c|c|c|c|}
\hline Sample & KL-03 & KL-05 & KL-09 & KL-12 & KL-28 & KL-45 & KL-06 & KL-40 & KL-34 & KL-35 & KL-19 & KL-15 \\
\hline Group & KL-I & KL-I & KL-I & KL-I & KL-II & KL-II & KL-III & KL-III & KL-III & KL-III & KL-III & KL-III \\
\hline $\begin{array}{l}\text { Lava Flow / } \\
\text { Lava Dome / } \\
\text { Cone }\end{array}$ & Bulochka & Bulochka & Novograblenov & Novograblenov & Maleev & Tuila & Belyankin & Bilyukai & Lepeshka & Lepeshka & Menyailov & Tsirk \\
\hline \multicolumn{13}{|c|}{ Major elements determined by XRF (wt \%) } \\
\hline $\mathrm{SiO}_{2}$ & 51.82 & 52.10 & 52.42 & 52.35 & 53.46 & 53.13 & 54.33 & 53.74 & 53.17 & 53.26 & 52.82 & 52.85 \\
\hline $\mathrm{TiO}_{2}$ & 0.83 & 0.81 & 0.84 & 0.83 & 0.84 & 0.90 & 1.01 & 0.96 & 1.05 & 1.05 & 0.97 & 0.92 \\
\hline $\mathrm{Al}_{2} \mathrm{O}_{3}$ & 13.34 & 13.32 & 13.92 & 13.72 & 14.34 & 14.16 & 16.81 & 15.17 & 15.98 & 16.57 & 15.34 & 15.73 \\
\hline FeOtot & 8.85 & 8.84 & 8.74 & 8.87 & 8.54 & 8.68 & 8.71 & 8.74 & 9.30 & 8.93 & 8.93 & 8.98 \\
\hline $\mathrm{MnO}$ & 0.16 & 0.16 & 0.16 & 0.16 & 0.15 & 0.16 & 0.16 & 0.16 & 0.17 & 0.16 & 0.16 & 0.16 \\
\hline $\mathrm{MgO}$ & 11.86 & 11.67 & 10.74 & 10.91 & 9.54 & 9.20 & 6.00 & 7.85 & 7.27 & 6.72 & 8.43 & 8.26 \\
\hline $\mathrm{CaO}$ & 10.27 & 10.17 & 10.12 & 10.09 & 9.64 & 10.13 & 8.50 & 9.43 & 9.32 & 9.22 & 9.73 & 9.48 \\
\hline $\mathrm{Na}_{2} \mathrm{O}$ & 2.17 & 2.17 & 2.28 & 2.29 & 2.56 & 2.54 & 3.30 & 2.77 & 2.75 & 3.16 & 2.74 & 2.76 \\
\hline $\mathrm{K}_{2} \mathrm{O}$ & 0.58 & 0.64 & 0.66 & 0.65 & 0.79 & 0.95 & 1.00 & 1.01 & 0.82 & 0.75 & 0.72 & 0.73 \\
\hline $\mathrm{P}_{2} \mathrm{O}_{5}$ & 0.12 & 0.12 & 0.13 & 0.13 & 0.14 & 0.16 & 0.18 & 0.17 & 0.16 & 0.18 & 0.16 & 0.14 \\
\hline Mg\# & 0.70 & 0.70 & 0.69 & 0.69 & 0.67 & 0.65 & 0.55 & 0.62 & 0.58 & 0.57 & 0.63 & 0.62 \\
\hline
\end{tabular}

Trace elements determined by XRF (ppm)

\begin{tabular}{|c|c|c|c|c|c|c|c|c|c|c|c|c|}
\hline $\mathrm{Ba}$ & 208 & 228 & 231 & 233 & 283 & 307 & 389 & 340 & 316 & 234 & 241 & 248 \\
\hline $\mathrm{Ce}$ & 5 & 9 & 7 & 13 & 12 & 5 & 14 & 12 & 14 & 16 & 17 & 17 \\
\hline $\mathrm{Cr}$ & 760 & 746 & 660 & 644 & 489 & 432 & 101 & 286 & 146 & 146 & 322 & 260 \\
\hline Ga & 14 & 14 & 14 & 14 & 15 & 15 & 18 & 16 & 17 & 17 & 16 & 16 \\
\hline $\mathrm{Nb}$ & 2.2 & 1.2 & 2.5 & 1.8 & 1.9 & 1.9 & 2.8 & 2.5 & 2.7 & 3.0 & 2.1 & 3.9 \\
\hline $\mathrm{Ni}$ & 186 & 181 & 160 & 162 & 138 & 103 & 39 & 74 & 39 & 48 & 87 & 83 \\
\hline $\mathrm{Pb}$ & 3.4 & 1.3 & 2.6 & 3.3 & 2.4 & 2.5 & 4.2 & 3.8 & 3.8 & 1.8 & 2.7 & 2.7 \\
\hline $\mathrm{Rb}$ & 10 & 10 & 12 & 10 & 12 & 15 & 17 & 16 & 13 & 11 & 11 & 12 \\
\hline Sc & 35 & 37 & 35 & 35 & 31 & 36 & 28 & 34 & 35 & 30 & 34 & 33 \\
\hline $\mathrm{Sr}$ & 242 & 239 & 253 & 249 & 308 & 319 & 319 & 322 & 306 & 308 & 286 & 299 \\
\hline Th & 1.8 & 2.1 & 3.6 & 1.5 & 1.5 & 1.3 & 2.6 & 2.9 & 2.6 & 2.4 & 1.5 & 1.0 \\
\hline V & 236 & 243 & 239 & 242 & 234 & 251 & 266 & 260 & 281 & 257 & 246 & 254 \\
\hline Y & 17 & 18 & 18 & 18 & 17 & 18 & 22 & 20 & 23 & 22 & 20 & 19 \\
\hline $\mathrm{Zr}$ & 66 & 67 & 71 & 70 & 74 & 72 & 94 & 82 & 83 & 95 & 83 & 76 \\
\hline
\end{tabular}

Trace elements determined by solution ICP-MS (ppm)

\begin{tabular}{|c|c|c|c|c|c|c|c|c|c|c|c|}
\hline & & & & & & & & & & & \\
\hline $\mathrm{Li}$ & 6.89 & 6.61 & 7.57 & 8.11 & 8.21 & 10.55 & 11.58 & 10.14 & 8.74 & 8.77 & 8.72 \\
\hline $\mathrm{Be}$ & 0.36 & 0.40 & 0.41 & 0.45 & 0.46 & 0.57 & 0.62 & 0.49 & 0.56 & 0.54 & 0.50 \\
\hline $\mathrm{Rb}$ & 7.67 & 8.41 & 9.07 & 8.96 & 10.82 & 13.08 & 14.07 & 13.20 & 11.96 & 9.39 & 9.42 \\
\hline Y & 15.26 & 15.53 & 16.31 & 16.25 & 14.52 & 16.71 & 20.21 & 18.08 & 20.79 & 20.09 & 17.67 \\
\hline $\mathrm{Zr}$ & 58.25 & 57.10 & 62.08 & 60.86 & 63.01 & 67.93 & 83.33 & 71.01 & 77.89 & 84.99 & 74.44 \\
\hline $\mathrm{Nb}$ & 1.24 & 1.27 & 1.33 & 1.30 & 1.37 & 1.47 & 1.79 & 1.62 & 1.85 & 1.96 & 1.68 \\
\hline $\mathrm{Sb}$ & 0.13 & 0.14 & 0.16 & 0.15 & 0.17 & 0.15 & 0.16 & 0.19 & 0.22 & 0.14 & 0.17 \\
\hline Cs & 0.29 & 0.34 & 0.35 & 0.32 & 0.38 & 0.44 & 0.51 & 0.42 & 0.46 & 0.17 & 0.34 \\
\hline $\mathrm{Ba}$ & 202 & 194 & 220 & 218 & 253 & 306 & 351 & 281 & 286 & 215 & 241 \\
\hline La & 3.73 & 3.95 & 4.14 & 4.14 & 4.79 & 5.56 & 6.25 & 5.71 & 5.35 & 5.48 & 4.86 \\
\hline $\mathrm{Ce}$ & 9.91 & 10.22 & 10.76 & 10.86 & 12.02 & 13.55 & 15.50 & 14.05 & 13.70 & 14.19 & 12.68 \\
\hline $\operatorname{Pr}$ & 1.55 & 1.60 & 1.68 & 1.70 & 1.82 & 2.06 & 2.38 & 2.15 & 2.12 & 2.17 & 1.94 \\
\hline $\mathrm{Nd}$ & 7.81 & 8.01 & 8.18 & 8.25 & 8.79 & 9.86 & 11.41 & 10.39 & 10.35 & 10.61 & 9.64 \\
\hline $\mathrm{Sm}$ & 2.22 & 2.27 & 2.39 & 2.39 & 2.38 & 2.68 & 3.08 & 2.80 & 2.99 & 2.99 & 2.68 \\
\hline $\mathrm{Eu}$ & 0.76 & 0.77 & 0.79 & 0.77 & 0.80 & 0.90 & 1.02 & 0.92 & 0.99 & 1.01 & 0.89 \\
\hline Gd & 2.65 & 2.67 & 2.73 & 2.76 & 2.67 & 3.02 & 3.46 & 3.17 & 3.44 & 3.46 & 3.04 \\
\hline $\mathrm{Tb}$ & 0.44 & 0.45 & 0.48 & 0.47 & 0.45 & 0.50 & 0.59 & 0.54 & 0.59 & 0.58 & 0.52 \\
\hline Dy & 2.85 & 2.91 & 2.95 & 3.00 & 2.74 & 3.10 & 3.76 & 3.39 & 3.77 & 3.66 & 3.28 \\
\hline Ho & 0.60 & 0.60 & 0.61 & 0.61 & 0.55 & 0.64 & 0.77 & 0.70 & 0.78 & 0.76 & 0.68 \\
\hline $\mathrm{Er}$ & 1.59 & 1.59 & 1.68 & 1.66 & 1.49 & 1.73 & 2.10 & 1.85 & 2.16 & 2.05 & 1.82 \\
\hline Tm & 0.25 & 0.25 & 0.26 & 0.26 & 0.23 & 0.26 & 0.32 & 0.30 & 0.33 & 0.33 & 0.29 \\
\hline $\mathrm{Yb}$ & 1.63 & 1.63 & 1.73 & 1.75 & 1.55 & 1.74 & 2.15 & 1.93 & 2.18 & 2.15 & 1.89 \\
\hline Lu & 0.24 & 0.25 & 0.26 & 0.27 & 0.23 & 0.26 & 0.33 & 0.29 & 0.33 & 0.32 & 0.29 \\
\hline Hf & 1.60 & 1.66 & 1.72 & 1.72 & 1.78 & 1.84 & 2.32 & 2.02 & 2.10 & 2.29 & 2.00 \\
\hline Тa & 0.12 & 0.13 & 0.14 & 0.13 & 0.15 & 0.14 & 0.19 & 0.16 & 0.19 & 0.19 & 0.17 \\
\hline $\mathrm{Tl}$ & 0.05 & 0.03 & 0.04 & 0.05 & 0.06 & 0.08 & 0.07 & 0.04 & 0.08 & 0.05 & 0.05 \\
\hline $\mathrm{Pb}$ & 1.97 & 2.11 & 2.17 & 2.19 & 3.28 & 2.74 & 3.15 & 2.84 & 2.68 & 1.84 & 2.31 \\
\hline Th & 0.36 & 0.40 & 0.43 & 0.44 & 0.55 & 0.64 & 0.71 & 0.63 & 0.63 & 0.50 & 0.47 \\
\hline U & 0.24 & 0.26 & 0.28 & 0.28 & 0.38 & 0.37 & 0.44 & 0.38 & 0.42 & 0.31 & 0.32 \\
\hline TIMS isotope & tios & & & & & & & & & & \\
\hline${ }^{87} \mathrm{Sr} /{ }^{86} \mathrm{Sr}$ & 0.70353 & & 0.70356 & & & 0.70350 & 0.70371 & & 0.70367 & & \\
\hline${ }^{143} \mathrm{Nd} /{ }^{144} \mathrm{Nd}$ & 0.51313 & & 0.51312 & & & 0.51312 & 0.51311 & & 0.51308 & & \\
\hline${ }^{206} \mathrm{~Pb} /{ }^{204} \mathrm{~Pb}$ & 18.291 & & & & & 18.290 & & & 18.303 & & \\
\hline${ }^{207} \mathrm{~Pb} /{ }^{204} \mathrm{~Pb}$ & 15.504 & & & & & 15.492 & & & 15.505 & & \\
\hline${ }^{208} \mathrm{~Pb} /{ }^{204} \mathrm{~Pb}$ & 37.994 & & & & & 37.956 & & & 38.007 & & \\
\hline
\end{tabular}

Notes: LD - lava dome. 


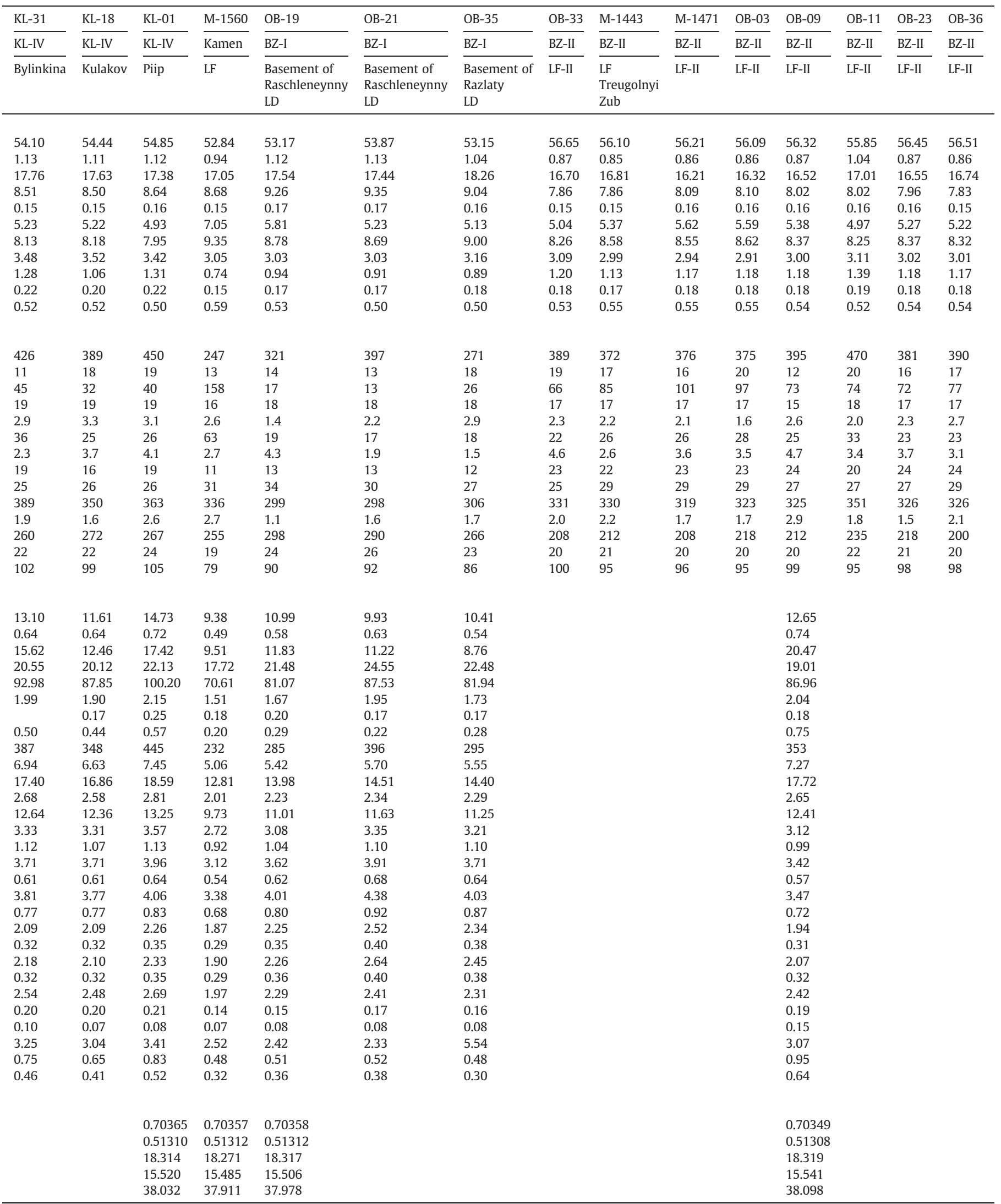


Table 3 (continued)

\begin{tabular}{|c|c|c|c|c|c|c|c|c|c|c|c|c|c|}
\hline Sample & OB-27 & OB-46 & OB-47 & OB-48 & OB-04 & OB-42 & OB-43 & Bez97-2 & Bez97-3 & Bez97-4 & Bez-B2 & Bez-B3 & OB-10 \\
\hline Group & BZ-II & BZ-III & BZ-III & BZ-III & BZ-III & BZ-III & BZ-III & BZ-III & BZ-III & BZ-III & BZ-III & BZ-III & BZ-III \\
\hline $\begin{array}{l}\text { Lava Flow / } \\
\text { Lava Dome / } \\
\text { Cone }\end{array}$ & LF-IV & $\begin{array}{l}\text { Plotina } \\
\text { LD }\end{array}$ & $\begin{array}{l}\text { Plotina } \\
\text { LD }\end{array}$ & $\begin{array}{l}\text { Plotina } \\
\text { LD }\end{array}$ & $\begin{array}{l}\text { Stupenchaty } \\
\text { LD }\end{array}$ & $\begin{array}{l}\text { Stupenchaty } \\
\text { LD }\end{array}$ & $\begin{array}{l}\text { Stupenchaty } \\
\text { LD }\end{array}$ & LF-1997 & LF-1997 & LF-1997 & LF-1997 & LF-1997 & LF-II \\
\hline \multicolumn{14}{|c|}{ Major elements determined by XRF (wt \%) } \\
\hline $\mathrm{SiO}_{2}$ & 56.25 & 57.06 & 57.00 & 57.26 & 57.26 & 57.50 & 57.47 & 57.85 & 58.37 & 57.51 & 57.69 & 57.72 & 57.18 \\
\hline $\mathrm{TiO}_{2}$ & 0.86 & 0.81 & 0.81 & 0.79 & 0.81 & 0.82 & 0.83 & 0.77 & 0.73 & 0.77 & 0.78 & 0.77 & 0.93 \\
\hline $\mathrm{Al}_{2} \mathrm{O}_{3}$ & 16.44 & 18.08 & 18.16 & 17.98 & 18.29 & 18.19 & 18.17 & 17.84 & 17.77 & 17.84 & 17.89 & 17.97 & 17.48 \\
\hline FeOtot & 7.98 & 7.76 & 7.72 & 7.85 & 7.76 & 7.62 & 7.51 & 7.41 & 7.17 & 7.51 & 7.33 & 7.24 & 7.57 \\
\hline $\mathrm{MnO}$ & 0.15 & 0.16 & 0.16 & 0.16 & 0.16 & 0.16 & 0.16 & 0.14 & 0.14 & 0.15 & 0.14 & 0.14 & 0.15 \\
\hline $\mathrm{MgO}$ & 5.48 & 3.85 & 3.81 & 3.72 & 3.44 & 3.43 & 3.65 & 3.84 & 3.71 & 4.01 & 3.95 & 3.87 & 4.22 \\
\hline $\mathrm{CaO}$ & 8.51 & 7.46 & 7.53 & 7.42 & 7.31 & 7.31 & 7.29 & 7.49 & 7.37 & 7.66 & 7.60 & 7.61 & 7.66 \\
\hline $\mathrm{Na}_{2} \mathrm{O}$ & 2.98 & 3.47 & 3.47 & 3.45 & 3.52 & 3.57 & 3.54 & 3.25 & 3.31 & 3.17 & 3.26 & 3.28 & 3.22 \\
\hline $\mathrm{K}_{2} \mathrm{O}$ & 1.16 & 1.11 & 1.11 & 1.13 & 1.21 & 1.18 & 1.16 & 1.24 & 1.27 & 1.22 & 1.21 & 1.23 & 1.39 \\
\hline $\mathrm{P}_{2} \mathrm{O}_{5}$ & 0.18 & 0.23 & 0.23 & 0.23 & 0.23 & 0.23 & 0.22 & 0.17 & 0.17 & 0.17 & 0.17 & 0.17 & 0.19 \\
\hline Mg\# & 0.55 & 0.47 & 0.47 & 0.46 & 0.44 & 0.45 & 0.46 & 0.48 & 0.48 & 0.49 & 0.49 & 0.49 & 0.50 \\
\hline \multicolumn{14}{|c|}{ Trace elements determined by XRF (ppm) } \\
\hline $\mathrm{Ba}$ & 386 & 368 & 357 & 369 & 370 & 371 & 357 & 393 & 404 & 382 & 385 & 385 & 523 \\
\hline $\mathrm{Ce}$ & 18 & 17 & 27 & 20 & 22 & 26 & 19 & 18 & 15 & 16 & 18 & 18 & 18 \\
\hline $\mathrm{Cr}$ & 93 & 26 & 22 & 29 & 8 & 13 & 14 & 20 & 21 & 24 & 22 & 19 & 25 \\
\hline $\mathrm{Ga}$ & 16 & 19 & 18 & 19 & 19 & 19 & 18 & 17 & 17 & 17 & 17 & 18 & 17 \\
\hline $\mathrm{Nb}$ & 2.7 & 2.9 & 3.2 & 4.0 & 2.9 & 3.1 & 3.9 & 2.9 & 2.9 & 1.6 & 2.1 & 2.2 & 2.9 \\
\hline $\mathrm{Ni}$ & 27 & 11 & 11 & 13 & 7 & 7 & 10 & 17 & 18 & 17 & 15 & 14 & 25 \\
\hline $\mathrm{Pb}$ & 3.3 & 2.4 & 3.3 & 2.5 & 3.1 & 3.2 & 4.4 & 4.0 & 4.9 & 4.3 & 3.6 & 4.1 & 3.6 \\
\hline $\mathrm{Rb}$ & 23 & 19 & 19 & 19 & 20 & 20 & 21 & 23 & 23 & 22 & 22 & 22 & 21 \\
\hline Sc & 28 & 19 & 18 & 19 & 19 & 19 & 20 & 22 & 23 & 24 & 22 & 22 & 22 \\
\hline $\mathrm{Sr}$ & 322 & 349 & 351 & 353 & 344 & 347 & 347 & 331 & 334 & 327 & 330 & 331 & 356 \\
\hline Th & 3.2 & 2.2 & 2.0 & 1.4 & 1.8 & 0.9 & 2.0 & 2.4 & 1.3 & 2.3 & 1.3 & 1.5 & 2.5 \\
\hline V & 208 & 161 & 165 & 159 & 166 & 156 & 169 & 194 & 174 & 189 & 203 & 195 & 215 \\
\hline Y & 21 & 22 & 22 & 23 & 23 & 22 & 23 & 18 & 19 & 19 & 18 & 19 & 21 \\
\hline $\mathrm{Zr}$ & 97 & 113 & 112 & 115 & 114 & 114 & 112 & 98 & 100 & 96 & 98 & 97 & 95 \\
\hline \multicolumn{14}{|c|}{ Trace elements determined by solution ICP-MS (ppm) } \\
\hline $\mathrm{Li}$ & 10.72 & 13.98 & & & & 13.40 & & 14.86 & & & & & 17.42 \\
\hline $\mathrm{Be}$ & 0.68 & 0.76 & & & & 0.80 & & 0.65 & & & & & 0.71 \\
\hline $\mathrm{Rb}$ & 21.22 & 16.73 & & & & 17.10 & & 18.74 & & & & & 14.93 \\
\hline Y & 18.68 & 20.05 & & & & 20.30 & & 16.35 & & & & & 17.86 \\
\hline $\mathrm{Zr}$ & 87.33 & 107.10 & & & & 104.20 & & 86.15 & & & & & 91.36 \\
\hline $\mathrm{Nb}$ & 1.98 & 3.07 & & & & 3.11 & & 1.75 & & & & & 2.02 \\
\hline $\mathrm{Sb}$ & 0.17 & 0.15 & & & & 0.23 & & 0.27 & & & & & 0.31 \\
\hline Cs & 0.71 & 0.51 & & & & 0.54 & & 0.71 & & & & & 0.59 \\
\hline $\mathrm{Ba}$ & 369 & 329 & & & & 320 & & 359 & & & & & 489 \\
\hline $\mathrm{La}$ & 7.18 & 8.63 & & & & 8.55 & & 7.17 & & & & & 6.41 \\
\hline $\mathrm{Ce}$ & 17.44 & 20.92 & & & & 20.69 & & 16.87 & & & & & 15.30 \\
\hline $\operatorname{Pr}$ & 2.57 & 3.06 & & & & 3.02 & & 2.41 & & & & & 2.42 \\
\hline $\mathrm{Nd}$ & 12.14 & 14.13 & & & & 14.04 & & 10.86 & & & & & 11.23 \\
\hline $\mathrm{Sm}$ & 3.04 & 3.49 & & & & 3.41 & & 2.71 & & & & & 2.98 \\
\hline $\mathrm{Eu}$ & 0.98 & 1.15 & & & & 1.11 & & 0.90 & & & & & 0.97 \\
\hline Gd & 3.39 & 3.77 & & & & 3.68 & & 2.90 & & & & & 3.24 \\
\hline $\mathrm{Tb}$ & 0.55 & 0.62 & & & & 0.60 & & 0.49 & & & & & 0.55 \\
\hline Dy & 3.39 & 3.69 & & & & 3.70 & & 2.99 & & & & & 3.38 \\
\hline Ho & 0.70 & 0.74 & & & & 0.78 & & 0.62 & & & & & 0.69 \\
\hline $\mathrm{Er}$ & 1.93 & 2.03 & & & & 2.10 & & 1.70 & & & & & 1.88 \\
\hline $\mathrm{Tm}$ & 0.30 & 0.32 & & & & 0.34 & & 0.28 & & & & & 0.30 \\
\hline $\mathrm{Yb}$ & 2.04 & 2.12 & & & & 2.24 & & 1.84 & & & & & 1.95 \\
\hline Lu & 0.31 & 0.34 & & & & 0.36 & & 0.29 & & & & & 0.31 \\
\hline $\mathrm{Hf}$ & 2.35 & 2.78 & & & & 2.85 & & 2.33 & & & & & 2.44 \\
\hline Ta & 0.19 & 0.29 & & & & 0.28 & & 0.17 & & & & & 0.24 \\
\hline $\mathrm{Tl}$ & 0.08 & 0.12 & & & & 0.13 & & 0.15 & & & & & 0.11 \\
\hline $\mathrm{Pb}$ & 2.79 & 2.43 & & & & 2.69 & & 3.43 & & & & & 3.46 \\
\hline Th & 0.91 & 0.85 & & & & 0.86 & & 1.00 & & & & & 0.67 \\
\hline $\mathrm{U}$ & 0.61 & 0.52 & & & & 0.55 & & 0.67 & & & & & 0.44 \\
\hline \multicolumn{14}{|c|}{ TIMS isotope ratios } \\
\hline${ }^{87} \mathrm{Sr} /{ }^{86} \mathrm{Sr}$ & & 0.70354 & & & & 0.70353 & & 0.70353 & & & & & \\
\hline${ }^{143} \mathrm{Nd} /{ }^{144} \mathrm{Nd}$ & & 0.51310 & & & & 0.51311 & & 0.51313 & & & & & \\
\hline \multicolumn{14}{|l|}{${ }^{206} \mathrm{~Pb} /{ }^{204} \mathrm{~Pb}$} \\
\hline \multicolumn{14}{|l|}{${ }^{207} \mathrm{~Pb} /{ }^{204} \mathrm{~Pb}$} \\
\hline${ }^{208} \mathrm{~Pb} /{ }^{204} \mathrm{~Pb}$ & & & & & & & & & & & & & \\
\hline
\end{tabular}




\begin{tabular}{|c|c|c|c|c|c|c|c|c|c|c|c|c|}
\hline OB-30 & OB-25 & OB-31 & OB-32 & OB-01 & OB-02 & M-1461 & OB-16 & OB-17 & M-1412 & OB-45 & OB-41 & Bez97-1 \\
\hline BZ-III & BZ-III & BZ-IV & BZ-IV & BZ-IV & BZ-IV & BZ-IV & BZ-IV & BZ-IV & BZ-IV & BZ-IV & BZ-IV & BZ-IV \\
\hline LF-II & LF-IV & $\begin{array}{l}\text { Dvuglavy } \\
\text { LD }\end{array}$ & $\begin{array}{l}\text { Dvuglavy } \\
\text { LD }\end{array}$ & $\begin{array}{l}\text { Expeditsii } \\
\text { LD }\end{array}$ & $\begin{array}{l}\text { Expeditsii } \\
\text { LD }\end{array}$ & $\begin{array}{l}\text { Lokhmaty } \\
\text { LD }\end{array}$ & $\begin{array}{l}\text { Lokhmaty } \\
\text { LD }\end{array}$ & $\begin{array}{l}\text { Lokhmaty } \\
\text { LD }\end{array}$ & $\begin{array}{l}\text { Plotina } \\
\text { LD }\end{array}$ & $\begin{array}{l}\text { Plotina } \\
\text { LD }\end{array}$ & $\begin{array}{l}\text { Stupenchaty } \\
\text { LD }\end{array}$ & LF-1956 \\
\hline 56.78 & 58.70 & 60.13 & 61.44 & 61.66 & 61.54 & 63.66 & 63.87 & 63.36 & 60.54 & 59.54 & 60.16 & 62.88 \\
\hline 0.76 & 0.76 & 0.67 & 0.55 & 0.62 & 0.62 & 0.52 & 0.51 & 0.55 & 0.62 & 0.66 & 0.68 & 0.60 \\
\hline 18.66 & 17.69 & 16.90 & 18.07 & 17.26 & 17.14 & 17.03 & 17.03 & 16.99 & 17.88 & 18.41 & 18.06 & 16.30 \\
\hline 8.34 & 6.97 & 6.28 & 6.11 & 5.87 & 5.86 & 5.22 & 5.08 & 5.28 & 6.32 & 6.68 & 6.62 & 5.56 \\
\hline 0.18 & 0.13 & 0.13 & 0.15 & 0.13 & 0.13 & 0.12 & 0.12 & 0.12 & 0.15 & 0.15 & 0.15 & 0.12 \\
\hline 2.97 & 3.66 & 3.78 & 1.93 & 3.04 & 3.15 & 2.27 & 2.23 & 2.49 & 2.73 & 2.57 & 2.61 & 3.20 \\
\hline 7.56 & 7.04 & 7.08 & 6.09 & 6.35 & 6.50 & 5.68 & 5.62 & 5.80 & 6.84 & 6.77 & 6.47 & 5.96 \\
\hline 3.51 & 3.55 & 3.36 & 3.97 & 3.49 & 3.46 & 3.60 & 3.65 & 3.56 & 3.42 & 3.76 & 3.78 & 3.52 \\
\hline 1.02 & 1.29 & 1.48 & 1.36 & 1.41 & 1.44 & 1.72 & 1.72 & 1.67 & 1.33 & 1.20 & 1.25 & 1.71 \\
\hline 0.24 & 0.19 & 0.18 & 0.32 & 0.17 & 0.17 & 0.17 & 0.17 & 0.17 & 0.18 & 0.25 & 0.24 & 0.16 \\
\hline 0.39 & 0.48 & 0.52 & 0.36 & 0.48 & 0.49 & 0.44 & 0.44 & 0.46 & 0.44 & 0.41 & 0.41 & 0.51 \\
\hline 291 & 397 & 511 & 377 & 490 & 499 & 612 & 622 & 604 & 434 & 398 & 395 & 618 \\
\hline 26 & 23 & 24 & 34 & 22 & 22 & 26 & 24 & 24 & 28 & 24 & 23 & 24 \\
\hline 1 & 44 & 70 & $<1$ & 53 & 65 & 24 & 24 & 24 & 13 & 11 & 5 & 69 \\
\hline 19 & 18 & 16 & 19 & 17 & 17 & 17 & 17 & 17 & 18 & 19 & 18 & 16 \\
\hline 2.8 & 2.2 & 2.2 & 3.2 & 2.3 & 3.6 & 2.8 & 3.2 & 3.7 & 3.3 & 3.3 & 2.8 & 2.5 \\
\hline 3 & 18 & 17 & 1 & 15 & 16 & 10 & 10 & 12 & 7 & 8 & 6 & 27 \\
\hline 2.3 & 4.4 & 5.2 & 3.8 & 4.0 & 4.4 & 6.5 & 5.3 & 4.2 & 4.7 & 4.5 & 1.7 & 5.6 \\
\hline 18 & 24 & 27 & 23 & 27 & 28 & 33 & 34 & 32 & 25 & 21 & 21 & 34 \\
\hline 13 & 17 & 20 & 10 & 14 & 15 & 12 & 12 & 13 & 15 & 15 & 14 & 18 \\
\hline 354 & 343 & 360 & 410 & 343 & 340 & 342 & 340 & 337 & 351 & 378 & 369 & 321 \\
\hline 1.4 & 1.7 & 3.8 & 2.5 & 2.2 & 2.2 & 2.4 & 3.1 & 2.6 & 1.4 & 2.5 & 2.1 & 2.0 \\
\hline 125 & 166 & 152 & 57 & 121 & 130 & 91 & 88 & 105 & 120 & 100 & 117 & 128 \\
\hline 24 & 20 & 17 & 21 & 16 & 16 & 17 & 16 & 17 & 19 & 21 & 21 & 18 \\
\hline 117 & 113 & 112 & 144 & 109 & 107 & 130 & 130 & 127 & 109 & 127 & 128 & 125 \\
\hline
\end{tabular}

\begin{tabular}{|c|c|c|c|c|}
\hline 15.18 & 18.24 & 18.37 & 20.16 & 12.53 \\
\hline 0.70 & 0.80 & 0.79 & 0.86 & 0.86 \\
\hline 14.42 & 22.01 & 23.38 & 30.70 & 18.52 \\
\hline 15.77 & 15.99 & 15.00 & 15.31 & 18.84 \\
\hline 106.60 & 110.50 & 72.95 & 121.50 & 102.00 \\
\hline 2.19 & 2.31 & 2.25 & 2.38 & 3.40 \\
\hline 0.28 & 0.80 & 0.26 & 0.40 & 0.21 \\
\hline 0.65 & 0.88 & 0.87 & 1.13 & 0.57 \\
\hline 374 & 534 & 453 & 569 & 376 \\
\hline 6.43 & 9.26 & 8.72 & 10.21 & 9.72 \\
\hline 15.31 & 21.38 & 19.84 & 22.80 & 23.18 \\
\hline 2.33 & 2.93 & 2.74 & 3.10 & 3.35 \\
\hline 10.58 & 12.68 & 11.86 & 12.96 & 15.18 \\
\hline 2.62 & 2.90 & 2.67 & 2.81 & 3.60 \\
\hline 0.86 & 0.91 & 0.86 & 0.85 & 1.20 \\
\hline 2.84 & 2.99 & 2.90 & 2.81 & 3.72 \\
\hline 0.48 & 0.48 & 0.45 & 0.44 & 0.59 \\
\hline 3.03 & 2.93 & 2.76 & 2.74 & 3.50 \\
\hline 0.63 & 0.61 & 0.56 & 0.56 & 0.70 \\
\hline 1.75 & 1.66 & 1.52 & 1.54 & 1.91 \\
\hline 0.28 & 0.27 & 0.25 & 0.26 & 0.31 \\
\hline 1.89 & 1.79 & 1.62 & 1.74 & 2.00 \\
\hline 0.30 & 0.28 & 0.26 & 0.29 & 0.33 \\
\hline 2.79 & 2.79 & 2.16 & 3.18 & 2.82 \\
\hline 0.28 & 0.24 & 0.23 & 0.24 & 0.31 \\
\hline 0.15 & 0.19 & 0.17 & 0.24 & 0.15 \\
\hline 3.84 & 4.83 & 4.31 & 5.51 & 3.00 \\
\hline 1.00 & 1.34 & 1.18 & 1.55 & 0.93 \\
\hline 0.54 & 0.86 & 0.81 & 1.10 & 0.55 \\
\hline \multirow{5}{*}{\multicolumn{2}{|c|}{$\begin{array}{l}0.70340 \\
0.51309\end{array}$}} & 0.70356 & 0.70354 & \\
\hline & & 0.51309 & 0.51305 & \\
\hline & & 18.294 & 18.269 & \\
\hline & & 15.503 & 15.475 & \\
\hline & & 37.977 & 37.885 & \\
\hline
\end{tabular}


Table 3 (continued)

\begin{tabular}{|c|c|c|c|c|c|c|c|c|c|c|c|c|}
\hline Sample & Bez97-5 & Bez97-6 & M-2020 & OB-08 & OB-26 & OB-44 & OB-06 & OB-29 & OB-34 & OB-05 & OB-37 & OB-38 \\
\hline Group & BZ-IV & BZ-IV & BZ-IV & BZ-IV & BZ-IV & BZ-IV & BZ-V & BZ-V & BZ-V & BZ-V & BZ-V & BZ-V \\
\hline $\begin{array}{l}\text { Lava Flow / } \\
\text { Lava Dome / } \\
\text { Cone }\end{array}$ & LF-1956 & LF-1956 & LF-1956 & LF-IV & LF-IV & LF-IV & $\begin{array}{l}\text { Gladkii } \\
\text { LD }\end{array}$ & $\begin{array}{l}\text { Gladkii } \\
\text { LD }\end{array}$ & $\begin{array}{l}\text { Greben } \\
\text { LD }\end{array}$ & $\begin{array}{l}\text { Niny } \\
\text { LD }\end{array}$ & $\begin{array}{l}\text { Pravilny } \\
\text { LD }\end{array}$ & $\begin{array}{l}\text { Pravilny } \\
\text { LD }\end{array}$ \\
\hline \multicolumn{13}{|c|}{ Major elements determined by XRF (wt \%) } \\
\hline $\mathrm{SiO}_{2}$ & 60.53 & 60.97 & 60.45 & 59.83 & 59.97 & 59.12 & 65.70 & 65.70 & 62.20 & 64.26 & 65.91 & 65.85 \\
\hline $\mathrm{TiO}_{2}$ & 0.63 & 0.61 & 0.61 & 0.71 & 0.69 & 0.71 & 0.42 & 0.41 & 0.60 & 0.45 & 0.41 & 0.41 \\
\hline $\mathrm{Al}_{2} \mathrm{O}_{3}$ & 17.78 & 17.70 & 18.03 & 16.82 & 16.93 & 16.48 & 17.71 & 17.74 & 17.00 & 18.09 & 17.65 & 17.62 \\
\hline FeOtot & 6.39 & 6.33 & 6.22 & 6.54 & 6.37 & 6.76 & 4.19 & 4.15 & 5.84 & 4.46 & 4.15 & 4.19 \\
\hline $\mathrm{MnO}$ & 0.14 & 0.14 & 0.14 & 0.13 & 0.13 & 0.13 & 0.14 & 0.14 & 0.13 & 0.14 & 0.14 & 0.14 \\
\hline $\mathrm{MgO}$ & 2.79 & 2.71 & 2.67 & 4.02 & 3.96 & 4.69 & 1.07 & 1.07 & 2.87 & 1.41 & 1.05 & 1.08 \\
\hline $\mathrm{CaO}$ & 6.83 & 6.60 & 6.96 & 6.87 & 6.94 & 7.28 & 4.79 & 4.81 & 6.18 & 5.24 & 4.73 & 4.70 \\
\hline $\mathrm{Na}_{2} \mathrm{O}$ & 3.41 & 3.39 & 3.45 & 3.39 & 3.36 & 3.24 & 4.33 & 4.35 & 3.53 & 4.01 & 4.35 & 4.32 \\
\hline $\mathrm{K}_{2} \mathrm{O}$ & 1.33 & 1.35 & 1.28 & 1.51 & 1.49 & 1.41 & 1.42 & 1.41 & 1.46 & 1.69 & 1.42 & 1.43 \\
\hline $\mathrm{P}_{2} \mathrm{O}_{5}$ & 0.17 & 0.17 & 0.17 & 0.17 & 0.17 & 0.18 & 0.25 & 0.23 & 0.18 & 0.25 & 0.19 & 0.25 \\
\hline Mg\# & 0.44 & 0.43 & 0.43 & 0.52 & 0.53 & 0.55 & 0.31 & 0.31 & 0.47 & 0.36 & 0.31 & 0.32 \\
\hline
\end{tabular}

Trace elements determined by XRF ( $\mathrm{ppm})$

\begin{tabular}{|c|c|c|c|c|c|c|c|c|c|c|c|c|}
\hline $\mathrm{Ba}$ & 439 & 452 & 441 & 525 & 517 & 496 & 433 & 437 & 500 & 516 & 430 & 445 \\
\hline $\mathrm{Ce}$ & 19 & 23 & 20 & 16 & 26 & 20 & 32 & 30 & 19 & 26 & 33 & 27 \\
\hline $\mathrm{Cr}$ & 13 & 22 & 12 & 67 & 61 & 115 & $<1$ & 1 & 47 & $<1$ & 6 & 2 \\
\hline Ga & 17 & 18 & 18 & 17 & 17 & 16 & 19 & 18 & 17 & 18 & 18 & 18 \\
\hline $\mathrm{Nb}$ & 2.7 & 3.4 & 3.0 & 3.5 & 2.1 & 2.8 & 3.9 & 3.8 & 2.9 & 2.8 & 3.6 & 3.6 \\
\hline $\mathrm{Ni}$ & 12 & 12 & 7 & 22 & 21 & 27 & 2 & 2 & 13 & 4 & 2 & 2 \\
\hline $\mathrm{Pb}$ & 4.5 & 5.7 & 4.7 & 3.5 & 4.6 & 4.4 & 3.7 & 4.7 & 4.8 & 5.7 & 4.7 & 4.2 \\
\hline $\mathrm{Rb}$ & 25 & 26 & 25 & 28 & 29 & 26 & 25 & 24 & 26 & 34 & 25 & 24 \\
\hline Sc & 14 & 14 & 15 & 20 & 17 & 23 & 5 & 4 & 15 & 5 & 6 & 4 \\
\hline $\mathrm{Sr}$ & 344 & 347 & 355 & 334 & 339 & 334 & 413 & 416 & 346 & 376 & 412 & 411 \\
\hline Th & 2.1 & 2.8 & 2.4 & 1.5 & 2.3 & 2.4 & 2.1 & 2.2 & 2.3 & 2.0 & 2.3 & 2.0 \\
\hline V & 132 & 118 & 116 & 161 & 151 & 172 & 14 & 14 & 111 & 41 & 12 & 18 \\
\hline Y & 19 & 17 & 18 & 18 & 18 & 18 & 17 & 18 & 17 & 18 & 17 & 17 \\
\hline $\mathrm{Zr}$ & 109 & 111 & 108 & 114 & 113 & 111 & 157 & 158 & 116 & 143 & 158 & 161 \\
\hline
\end{tabular}

Trace elements determined by solution ICP-MS (

\begin{tabular}{|c|c|c|c|}
\hline $\mathrm{Li}$ & 17.27 & 19.19 & 18.22 \\
\hline $\mathrm{Be}$ & 0.75 & 0.79 & 0.77 \\
\hline $\mathrm{Rb}$ & 21.78 & 22.22 & 22.59 \\
\hline Y & 17.18 & 16.34 & 14.75 \\
\hline $\mathrm{Zr}$ & 96.23 & 111.60 & 73.85 \\
\hline $\mathrm{Nb}$ & 2.06 & 2.29 & 2.29 \\
\hline $\mathrm{Sb}$ & 0.28 & 0.32 & 0.25 \\
\hline Cs & 0.81 & 0.90 & 0.85 \\
\hline $\mathrm{Ba}$ & 416 & 590 & 454 \\
\hline La & 8.39 & 9.16 & 8.88 \\
\hline $\mathrm{Ce}$ & 19.53 & 21.19 & 20.20 \\
\hline $\operatorname{Pr}$ & 2.75 & 2.89 & 2.78 \\
\hline $\mathrm{Nd}$ & 12.18 & 12.36 & 11.97 \\
\hline $\mathrm{Sm}$ & 2.92 & 2.91 & 2.77 \\
\hline $\mathrm{Eu}$ & 0.95 & 0.89 & 0.87 \\
\hline Gd & 3.10 & 3.03 & 2.77 \\
\hline $\mathrm{Tb}$ & 0.50 & 0.48 & 0.44 \\
\hline Dy & 3.13 & 2.94 & 2.62 \\
\hline Ho & 0.63 & 0.61 & 0.53 \\
\hline $\mathrm{Er}$ & 1.74 & 1.67 & 1.49 \\
\hline $\mathrm{Tm}$ & 0.28 & 0.26 & 0.24 \\
\hline $\mathrm{Yb}$ & 1.91 & 1.76 & 1.59 \\
\hline $\mathrm{Lu}$ & 0.30 & 0.28 & 0.26 \\
\hline $\mathrm{Hf}$ & 2.60 & 2.85 & 2.14 \\
\hline Ta & 0.20 & 0.22 & 0.22 \\
\hline $\mathrm{Tl}$ & 0.17 & 0.15 & 0.15 \\
\hline $\mathrm{Pb}$ & 4.22 & 4.73 & 4.03 \\
\hline Th & 1.15 & 1.39 & 1.17 \\
\hline $\mathrm{U}$ & 0.79 & 0.84 & 0.79 \\
\hline & & & \\
\hline${ }^{87} \mathrm{Sr}$ & & & 0.70362 \\
\hline${ }^{143} \mathrm{~N}$ & & & 0.51309 \\
\hline${ }^{206} \mathrm{P}$ & & & \\
\hline & & & \\
\hline & & & \\
\hline
\end{tabular}




\begin{tabular}{|c|c|c|c|c|c|c|c|c|c|c|}
\hline OB-39 & OB-40 & OB-12 & OB-13 & OB-14 & OB-15 & OB-18 & OB-20 & OB-24 & OB-07 & OB-28 \\
\hline BZ-V & BZ-V & BZ-V & BZ-V & BZ-V & BZ-V & BZ-V & BZ-V & BZ-V & BZ-V & BZ-V \\
\hline $\begin{array}{l}\text { Pravilny } \\
\text { LD }\end{array}$ & $\begin{array}{l}\text { Pravilny } \\
\text { LD }\end{array}$ & $\begin{array}{l}\text { Raschleneynny } \\
\text { LD }\end{array}$ & $\begin{array}{l}\text { Raschleneynny } \\
\text { LD }\end{array}$ & $\begin{array}{l}\text { Raschleneynny } \\
\text { LD }\end{array}$ & $\begin{array}{l}\text { Raschleneynny } \\
\text { LD }\end{array}$ & $\begin{array}{l}\text { Raschleneynny } \\
\text { LD }\end{array}$ & $\begin{array}{l}\text { Raschleneynny } \\
\text { LD }\end{array}$ & $\begin{array}{l}\text { Raschleneynny } \\
\text { LD }\end{array}$ & Zub LD & Zub LD \\
\hline 65.91 & 65.64 & 67.25 & 67.42 & 67.09 & 66.78 & 68.03 & 67.74 & 67.54 & 64.36 & 64.19 \\
\hline 0.41 & 0.41 & 0.37 & 0.36 & 0.39 & 0.39 & 0.34 & 0.35 & 0.36 & 0.44 & 0.44 \\
\hline 17.58 & 17.63 & 17.13 & 17.21 & 16.84 & 17.01 & 17.12 & 17.22 & 17.13 & 17.97 & 18.09 \\
\hline 4.15 & 4.29 & 3.56 & 3.44 & 3.99 & 3.95 & 3.19 & 3.24 & 3.46 & 4.50 & 4.43 \\
\hline 0.14 & 0.14 & 0.12 & 0.12 & 0.12 & 0.12 & 0.12 & 0.12 & 0.10 & 0.14 & 0.13 \\
\hline 1.07 & 1.10 & 1.05 & 0.97 & 1.22 & 1.24 & 0.87 & 0.92 & 1.05 & 1.37 & 1.38 \\
\hline 4.74 & 4.77 & 4.37 & 4.34 & 4.43 & 4.59 & 4.16 & 4.23 & 4.35 & 5.19 & 5.32 \\
\hline 4.30 & 4.34 & 4.03 & 4.03 & 3.82 & 3.84 & 4.03 & 4.04 & 3.92 & 4.10 & 4.11 \\
\hline 1.44 & 1.42 & 1.95 & 1.94 & 1.93 & 1.91 & 1.97 & 1.97 & 1.94 & 1.68 & 1.66 \\
\hline 0.25 & 0.25 & 0.17 & 0.17 & 0.18 & 0.17 & 0.16 & 0.17 & 0.15 & 0.24 & 0.24 \\
\hline 0.32 & 0.31 & 0.34 & 0.33 & 0.35 & 0.36 & 0.33 & 0.34 & 0.35 & 0.35 & 0.36 \\
\hline 435 & 447 & 641 & 640 & 650 & 641 & 644 & 633 & 638 & 517 & 505 \\
\hline 37 & 35 & 24 & 31 & 25 & 26 & 28 & 21 & 24 & 32 & 25 \\
\hline$<1$ & $<1$ & 3 & 2 & 2 & 2 & $<1$ & 8 & 1 & 5 & $<1$ \\
\hline 18 & 18 & 17 & 17 & 17 & 17 & 18 & 18 & 18 & 18 & 18 \\
\hline 4.5 & 3.5 & 2.6 & 4.5 & 3.4 & 3.5 & 3.3 & 4.1 & 4.3 & 4.0 & 3.7 \\
\hline 2 & 2 & 3 & 1 & 3 & 4 & 3 & 3 & 2 & 3 & 2 \\
\hline 4.7 & 5.4 & 6.8 & 7.9 & 6.0 & 6.5 & 7.0 & 8.0 & 6.9 & 5.6 & 4.3 \\
\hline 26 & 25 & 40 & 41 & 41 & 39 & 41 & 41 & 41 & 34 & 33 \\
\hline 5 & 5 & 7 & 8 & 7 & 8 & 5 & 5 & 5 & 7 & 7 \\
\hline 412 & 414 & 348 & 351 & 337 & 346 & 349 & 350 & 349 & 376 & 379 \\
\hline 2.1 & 2.2 & 2.0 & 2.0 & 2.3 & 1.4 & 2.4 & 2.5 & 2.4 & 3.6 & 2.5 \\
\hline 14 & 18 & 32 & 24 & 34 & 49 & 15 & 13 & 32 & 40 & 37 \\
\hline 18 & 19 & 16 & 15 & 15 & 15 & 15 & 15 & 15 & 18 & 18 \\
\hline 161 & 160 & 116 & 116 & 113 & 110 & 118 & 116 & 116 & 143 & 142 \\
\hline & 12.09 & & & & 24.81 & 25.71 & & & 20.81 & \\
\hline & 0.93 & & & & 0.98 & 1.18 & & & 1.06 & \\
\hline & 18.75 & & & & 35.91 & 38.62 & & & 30.93 & \\
\hline & 10.59 & & & & 12.97 & 12.63 & & & 16.92 & \\
\hline & 143.40 & & & & 43.74 & 72.78 & & & 144.00 & \\
\hline & 3.50 & & & & 2.99 & 3.00 & & & 3.14 & \\
\hline & 0.28 & & & & 0.23 & 0.44 & & & 0.37 & \\
\hline & 0.56 & & & & 1.01 & 1.19 & & & 0.97 & \\
\hline & 432 & & & & 515 & 662 & & & 549 & \\
\hline & 7.34 & & & & 9.84 & 10.55 & & & 11.41 & \\
\hline & 18.04 & & & & 22.70 & 23.82 & & & 26.73 & \\
\hline & 2.53 & & & & 3.09 & 3.14 & & & 3.62 & \\
\hline & 11.22 & & & & 12.90 & 12.98 & & & 15.10 & \\
\hline & 2.42 & & & & 2.78 & 2.71 & & & 3.25 & \\
\hline & 0.75 & & & & 0.84 & 0.79 & & & 0.99 & \\
\hline & 2.34 & & & & 2.72 & 2.51 & & & 3.17 & \\
\hline & 0.34 & & & & 0.41 & 0.38 & & & 0.49 & \\
\hline & 2.06 & & & & 2.45 & 2.21 & & & 2.99 & \\
\hline & 0.41 & & & & 0.48 & 0.46 & & & 0.60 & \\
\hline & 1.13 & & & & 1.30 & 1.19 & & & 1.67 & \\
\hline & 0.18 & & & & 0.21 & 0.19 & & & 0.28 & \\
\hline & 1.34 & & & & 1.42 & 1.31 & & & 1.96 & \\
\hline & 0.21 & & & & 0.23 & 0.21 & & & 0.31 & \\
\hline & 3.58 & & & & 1.53 & 2.11 & & & 3.50 & \\
\hline & 0.37 & & & & 0.34 & 0.32 & & & 0.34 & \\
\hline & 0.15 & & & & 0.25 & 0.09 & & & 0.24 & \\
\hline & 5.65 & & & & 6.00 & 6.18 & & & 5.90 & \\
\hline & 0.80 & & & & 1.30 & 1.35 & & & 1.43 & \\
\hline & 0.62 & & & & 0.70 & 1.01 & & & 0.96 & \\
\hline & 0.70362 & & & & 0.70357 & & & & 0.70353 & \\
\hline & 0.51311 & & & & 0.51310 & & & & 0.51310 & \\
\hline & & & & & 18.284 & & & & & \\
\hline & & & & & 15.472 & & & & & \\
\hline & & & & & 37.852 & & & & & \\
\hline
\end{tabular}



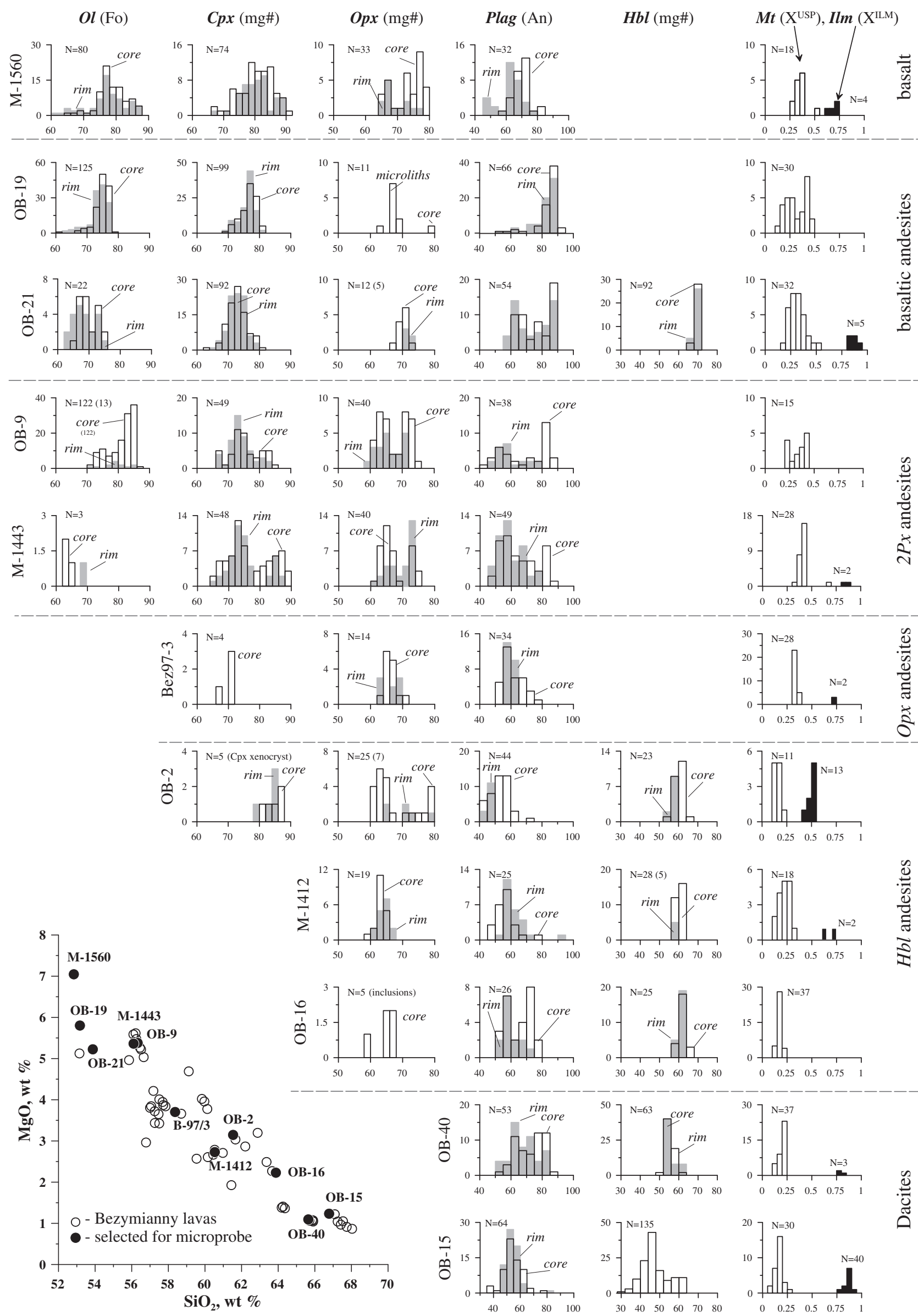

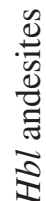
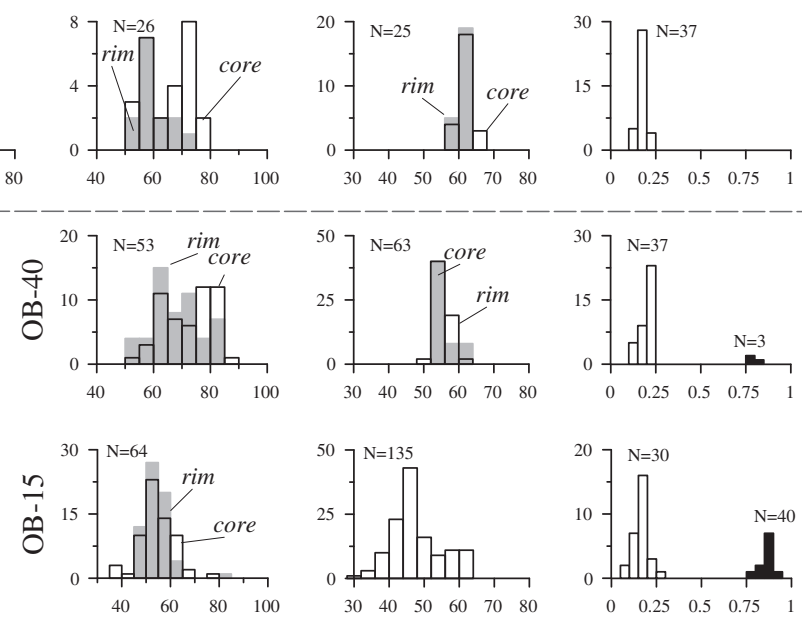
in $2 \mathrm{Px}$ andesites, and $\mathrm{An}_{80-75}$ and $\mathrm{An}_{60-58}$ in $\mathrm{Hbl}$ andesites and dacites (Fig. 3). Basaltic andesite OB-19 contains abundant Plag with the highest An content of as much as An ${ }_{92}$. Such a high-An Plag is rarely found in andesites and dacites.

Mt occurs in all lava types from basaltic andesite to dacite and its composition changes gradually with silica enrichment of the host lavas, showing a broad decrease in the ulvospinel component $\left(X^{\mathrm{USP}}\right)$ from $50 \mathrm{~mol} \%$ to $10 \mathrm{~mol} \%$.

\subsection{Major element compositions}

The lavas are classified as medium-K basalt, basaltic andesite, andesite, and dacite, displaying a calc-alkaline differentiation trend typical of island arc lavas (Fig. 4). The calc-alkaline affinity is more pronounced in Bezymianny lavas, whereas Kliuchevskoi basalt lavas exhibit a slight increase in $\mathrm{FeO} / \mathrm{MgO}$. Even so, the Kliuchevskoi-Bezymianny lavas span a continuum in compositional trend from $52 \mathrm{wt} . \%$ to $68 \mathrm{wt} . \% \mathrm{SiO}_{2}, 12$ to 0.9 wt.\% $\mathrm{MgO}$, and 0.6 to $1.9 \mathrm{wt} . \% \mathrm{~K}_{2} \mathrm{O}$; in general the trend shows a change of slope (or an inflection point) at $6 \mathrm{wt} . \% \mathrm{MgO}$ for some elements (e.g. $\mathrm{FeO}, \mathrm{Al}_{2} \mathrm{O}_{3}, \mathrm{TiO}_{2}$, Fig. 5). $\mathrm{Mg} \#$ of the lavas also decreases monotonically from 0.7 in HMB to 0.3 in dacite (Tables 2, 3). With the increase in $\mathrm{SiO}_{2}$ there is a systematic decrease in $\mathrm{CaO}$ and $\mathrm{MgO}$ coupled with an increase in $\mathrm{Na}_{2} \mathrm{O}$ and $\mathrm{K}_{2} \mathrm{O}$ (Fig. 5). If the lavas are considered collectively, the trends for $\mathrm{FeO}, \mathrm{Al}_{2} \mathrm{O}_{3}, \mathrm{TiO}_{2}, \mathrm{MnO}$, and $\mathrm{P}_{2} \mathrm{O}_{5}\left(\mathrm{P}_{2} \mathrm{O}_{5}\right.$ not shown) are segmented (e.g. when plotted against $\mathrm{MgO}$, Fig. 5). In Kliuchevskoi $\mathrm{HMB}$ to $\mathrm{HAB}, \mathrm{Al}_{2} \mathrm{O}_{3}, \mathrm{TiO}_{2}$, and $\mathrm{P}_{2} \mathrm{O}_{5}$ increase with decreasing $\mathrm{MgO}$, whereas $\mathrm{FeO}$ and $\mathrm{MnO}$ are almost constant up to 5-6 wt.\% MgO.

The transition from basaltic to intermediate lavas occurs at around 5-6 wt.\% MgO and it is characterized by a sharp inflection at which $\mathrm{TiO}_{2}$, FeO, and $\mathrm{MnO}$ start to decrease. This is accompanied by a complementary enrichment in $\mathrm{SiO}_{2} . \mathrm{Al}_{2} \mathrm{O}_{3}$ tends to be relatively constant after this inflection point. The observed inflection may correspond to the onset of crystallization of certain minerals. For example, the termination of $\mathrm{A}_{2} \mathrm{O}_{3}$ enrichment can be attributed to the onset of Plag crystallization. A strong depletion of $\mathrm{FeO}$ and $\mathrm{TiO}_{2}$ can be linked to the onset of $M t$ fractionation. This implies that Plag and Mt should coexist in Bezymianny melt from the earliest magma differentiation stages. On the other hand, strong $\mathrm{FeO}, \mathrm{TiO}_{2}, \mathrm{CaO}$, and $\mathrm{K}_{2} \mathrm{O}$ (and minor elements, see below) linear trends in Bezymianny lavas can be interpreted as a result of magma mixing between two end-member compositions, e.g. HABs and dacites. We will examine this possibility in a later section.

\subsection{Trace element compositions}

Trace element X-ray fluorescence (XRF) and inductively coupled plasma mass spectrometry (ICP-MS) analyses for lavas from Kliuchevskoi and Bezymianny are listed in Table 3 and are plotted against differentiation indices ( $\mathrm{MgO}$ and $\mathrm{K}_{2} \mathrm{O}$ in Figs. 6 and 7 respectively). Similar to major elements, compatible trace elements also display strong and systematic variations with respect to $\mathrm{MgO}$. Sharp boomerang-like inflections in $\mathrm{Cr}$, $\mathrm{Ni}, \mathrm{Sc}$, and $\mathrm{V}$ are shown at $\mathrm{MgO}=\sim 6 \mathrm{wt} . \%$. $\mathrm{Y}$ and $\mathrm{Yb}$ exhibit similar but smaller inflections (Fig. 6).

$\mathrm{Ni}$ and $\mathrm{Cr}$ behavior is strongly controlled by $\mathrm{Ol}$ and $\mathrm{Cpx}+\mathrm{Sp}$ crystallization, respectively (Kinzler et al., 1990; Righter et al., 2006). A weak Sc decrease in basalt and its stronger decrease in the evolved lavas can be attributed to the onset of $C p x$ fractionation in basaltic andesite, followed by subsequent fractionation of $\mathrm{Hbl}$ in andesites and dacites (Hart and Dunn, 1993; Sisson, 1994). V, Y, and Yb abundances first show a slight increase in basalts, followed by depletion in andesite and dacite compositions. A strong $\mathrm{V}$ decrease in the Bezymianny lavas denotes the onset of $M t$ crystallization (Luhr and Carmichael, 1980). The similar but less notable behavior of $\mathrm{Y}$ and $\mathrm{Yb}$ as well as other mid rare earth elements (MREE) to heavy REE (HREE) is explained by the onset of $\mathrm{Cpx}$ and/or $\mathrm{Hbl}$ fractional crystallization (Hart and Dunn, 1993; Adam and Green, 1994; Sisson, 1994; Dobosi and Jenner, 1999; Davidson et al., 2007). Sr and Ga increase almost linearly with subtle inflections at $\mathrm{MgO}=\sim 6 \mathrm{wt} . \%$. These inflections are likely controlled by the onset of Plag fractionation because these elements are compatible with Plag. Therefore these two elements behave similarly to $\mathrm{Al}_{2} \mathrm{O}_{3}$ (Bedard, 2006).

In contrast, incompatible trace elements demonstrate strong positive correlations with the index of differentiation (Fig. 7). Concentrations of all highly incompatible trace elements continuously increase by a factor of $\sim 3$ for $\mathrm{Be}, \mathrm{Zr}, \mathrm{Nb}, \mathrm{Ba}$, $\mathrm{La}$, Ta, and $\mathrm{Pb}$, factor of $\sim 4$ for $\mathrm{Li}$, Th, and $U$, and factor of $\sim 5$ for $\mathrm{Rb}$ during fractional crystallization of the primary HMB (Table 3, Fig. 7). A simple Rayleigh fractionation for the entire volcanic suite, from HMB to dacites, suggests that all geochemical variations found in the Kliuchevskoi-Bezymianny lavas can be explained by $\sim 70 \%$ crystal fractionation using HMB as the parental liquid composition. The differences in enrichment factor result in a weak inflection of the geochemical trends for some elements (e.g. Rb vs. $\mathrm{K}_{2} \mathrm{O}$ ). In addition, individual Kliuchevskoi and Bezymianny geochemical trends have somewhat different slopes for some elements (e.g. Li, Th in Fig. 7). In general, andesite-dacite Bezymianny lavas appear to inherit their geochemical characteristics with respect to many trace elements from Kliuchevskoi basalts (with some non-systematic outliers). However there is a group of elements which may indicate the existence of mantle source geochemical variability (e.g. $\mathrm{Nb}$ in Fig. 7; see discussion below).

Fig. 8 shows the REE patterns of Kliuchevskoi and Bezymianny lavas normalized to chondrite values (Sun and McDonough, 1989). The studied lavas have relatively low total REE contents (36-73 ppm) and light REE (LREE)-enriched patterns $\left((\mathrm{La} / \mathrm{Yb})_{\mathrm{n}}=1.5\right.$ to 5.8$)$ typical for island arc magmas. The most primitive HMB displays almost flat REE patterns with a weak HREE depletion. From HMB through HAB and further to basaltic andesite, REE patterns are nearly parallel but abundance increases systematically with progressive differentiation in all samples (Fig. 8a, b). In andesites (Fig. 8c, d, e), this parallel shift is also tilted and as a result the LREE are enriched, while MREE and HREE are slightly depleted. These "spoon"-shaped patterns develop continuously with differentiation and are most pronounced in dacites (Fig. 8d, e, f). The profound MREE-HREE depletion observed in $\mathrm{Hbl}$-bearing andesites (Fig. 8e) and dacites (Fig. 8f) indicates $\mathrm{Hbl}$ fractionation during crystallization (Adam and Green, 2003; Davidson et al., 2007; Brophy, 2008) in addition to the fractionation of apatite crystals (see Fig. 12 and relevant discussions below).

Although Plag is the prevailing phenocryst in the differentiated lavas, the lack of positive Eu anomalies suggests no Plag accumulation during magma differentiation (Crawford et al., 1987). All the calculated $\mathrm{Eu} / \mathrm{Eu}^{*}$ ratios, where $\mathrm{Eu}^{*}=(\mathrm{Sm} \cdot \mathrm{Gd})^{0.5}$, are below 1 with mean value $0.95 \pm 0.02$, indicating Plag fractionation rather than accumulation as suggested by $\mathrm{Al}_{2} \mathrm{O}_{3}, \mathrm{Sr}$, and $\mathrm{Ga}$ (Figs. 5, 6).

Mid-Ocean Ridge Basalt (MORB)-normalized multi-element plot patterns of the Kliuchevskoi and Bezymianny lavas are spiked and have characteristics typical of island arc magmas. They are enriched in $\mathrm{Li}, \mathrm{Th}, \mathrm{U}, \mathrm{Pb}$, and large ion lithophile elements (LILEs, such as Cs, Tl, $\mathrm{Rb}, \mathrm{Ba}, \mathrm{Sr}$, and $\mathrm{K}$ ) and depleted in high field strength elements (HFSEs, $\mathrm{Nb}$ and Ta, Fig. 9) relative to REE. With progressive silica enrichment in the rocks, all differentiated samples are also enriched in incompatible elements. Exceptions are (1) $\mathrm{Zr}$ and Hf concentrations which are low in

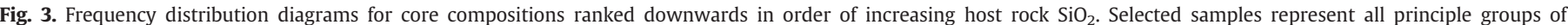

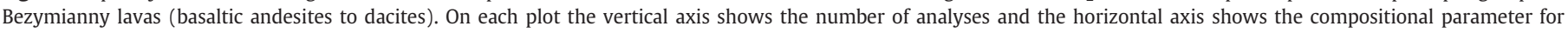

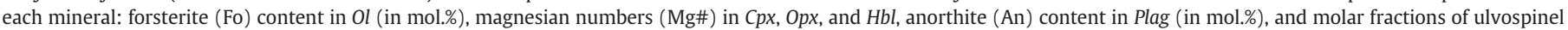
$\left(X^{\mathrm{USP}}\right)$ or ilmenite $\left(X^{\mathrm{ILM}}\right)$ components in titanomagnetite and $\mathrm{Ilm}$ respectively. Inset shows samples selected for phenocryst composition analysis on an $\mathrm{SiO}_{2}-\mathrm{MgO}$ plot. 

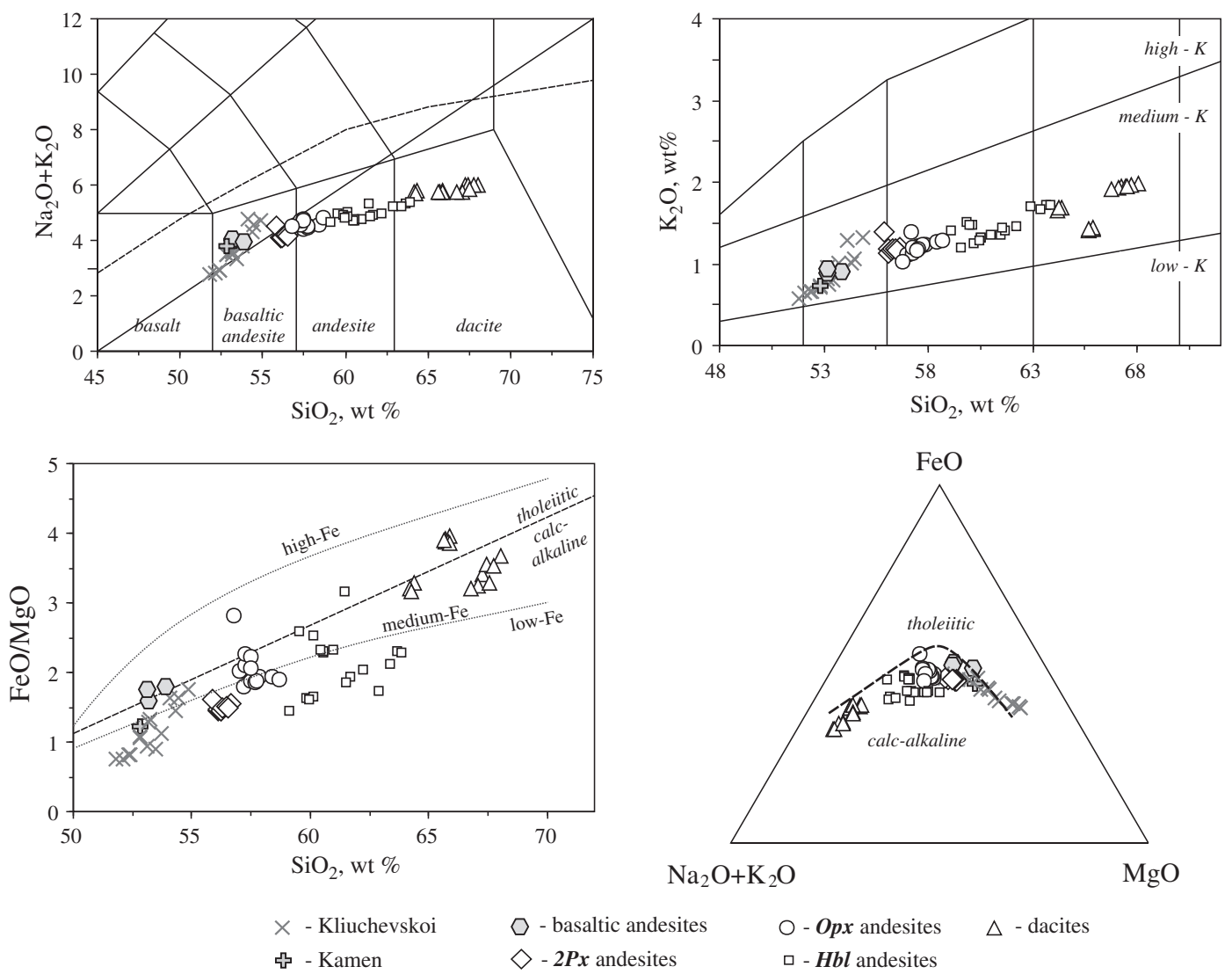

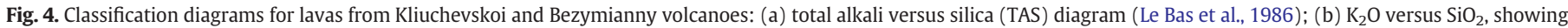

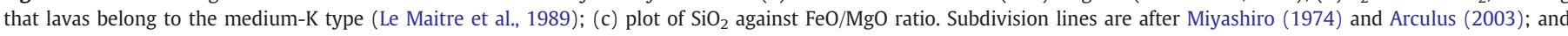
(d) AFM plot subdividing tholeiitic and calc-alkaline differentiation trends (Irvine and Baragar, 1971).

dacites probably due to zircon crystallization, and (2) Y, MREE, and HREE contents in andesites and dacites which are low due to $\mathrm{Hbl}$ and apatite crystallization as shown by REEs (see above, Fig. 8).

\subsection{1. $\mathrm{Sr}, \mathrm{Nd}$, and $\mathrm{Pb}$ isotopes}

The $\mathrm{Sr}, \mathrm{Nd}$, and $\mathrm{Pb}$ isotope ratios analyzed in selected samples are given in Table 3. Our new isotope data for the Kliuchevskoi and Bezymianny lavas are in good agreement with results obtained previously for CKD lavas in general and for the studied volcanoes in particular (Kersting, 1995; Kepezhinskas et al., 1997; Ozerov et al., 1997; Turner et al., 1998; Dorendorf et al., 2000; Churikova et al., 2001). The Nd isotope compositions of the Kliuchevskoi and Bezymianny lavas are within the range of depleted N-MORB mantle source. The radiogenic Sr and $\mathrm{Pb}$ isotope compositions indicate involvement of the $\mathrm{Sr}$ and $\mathrm{Pb}$ rich slab-derived fluids (or melts) in magma genesis (Kepezhinskas et al., 1997; Turner et al., 1998; Dorendorf et al., 2000; Churikova et al., 2001). Relatively unradiogenic Pb compositions of the lavas indicate only a small contribution by subducted sediments, and instead more involvement of $\mathrm{Pb}$ from altered oceanic crust in magma genesis (Kersting, 1995; Dorendorf et al., 2000; Churikova et al., 2001).

Lavas from both volcanoes are indistinguishable in terms of their $\mathrm{Sr}-\mathrm{Nd}-\mathrm{Pb}$ isotopic ratios and display non-systematic variations (Fig. 10a, b) within a very narrow range: ${ }^{87} \mathrm{Sr} /{ }^{86} \mathrm{Sr}=0.7034-0.7037$ and ${ }^{143} \mathrm{Nd} /{ }^{144} \mathrm{Nd}=0.51305-0.51313$. Similarly, Pb isotopes cannot resolve any details of lava source heterogeneity because they vary in the ranges ${ }^{206} \mathrm{~Pb} /{ }^{204} \mathrm{~Pb}=18.269-18.319,{ }^{207} \mathrm{~Pb} /{ }^{204} \mathrm{~Pb}=15.472-$ 15.541, and ${ }^{208} \mathrm{~Pb} /{ }^{204} \mathrm{~Pb}=37.852-38.098$. There are slight positive linear correlations between ${ }^{206} \mathrm{~Pb} /{ }^{204} \mathrm{~Pb},{ }^{207} \mathrm{~Pb} /{ }^{204} \mathrm{~Pb}$, and ${ }^{208} \mathrm{~Pb} /{ }^{204} \mathrm{~Pb}$. However, there is neither systematic variation between lavas from two volcanoes nor any age correlation (see Fig. 10 caption). Also, from basalts to dacites there are no systematic and significant correlations between $\mathrm{Sr}-\mathrm{Nd}-\mathrm{Pb}$ isotope ratios and differentiation indices (Fig. 10e, f). These isotope data preclude significant contributions of crustal material in the magma genesis of Kliuchevskoi and Bezymianny volcanoes.

\section{Discussion}

5.1. Mantle source: geochemical evidence for a common primary basalt source

Previous studies have demonstrated large variations of $\delta^{18} \mathrm{O}$ and ${ }^{87} \mathrm{Sr} /{ }^{86} \mathrm{Sr}$ and some trace element ratios in Kliuchevskoi rocks which cannot be explained by simple fractional crystallization and require crustal assimilation and/or variable mantle-derived parents (Dorendorf et al., 2000). Recently, Auer et al. (2009) confirmed extremely high $\delta^{18} \mathrm{O}$ in the Kliuchevskoi lavas and suggested a contribution by the hydrothermally altered lithospheric mantle. Portnyagin et al. (2007a, 2007b) studied melt inclusions in magnesian $\mathrm{Ol}$ and demonstrated slightly different geochemical characteristics for the CKD primary magmas in general and for the historic and pre-historic primitive melts beneath Kliuchevskoi Volcano in particular. Our new geochemical data are useful for assessing the geochemical variability of the Bezymianny and pre-Bezymianny eruptive products as well as their genetic relations to the Kliuchevskoi magmas.

As was described in Section 4.4.1, the Bezymianny lavas have isotopic compositions within the field of Kliuchevskoi rocks (Fig. 10) indicating derivation from a similar mantle source(s). This conclusion is emphasized by the similar behavior of incompatible elements (Fig. 7). In Fig. 11 trace element ratios are plotted against Li chosen as an index of differentiation. The strong positive correlation of $\mathrm{Li}$ with $\mathrm{K}_{2} \mathrm{O}$ in the 

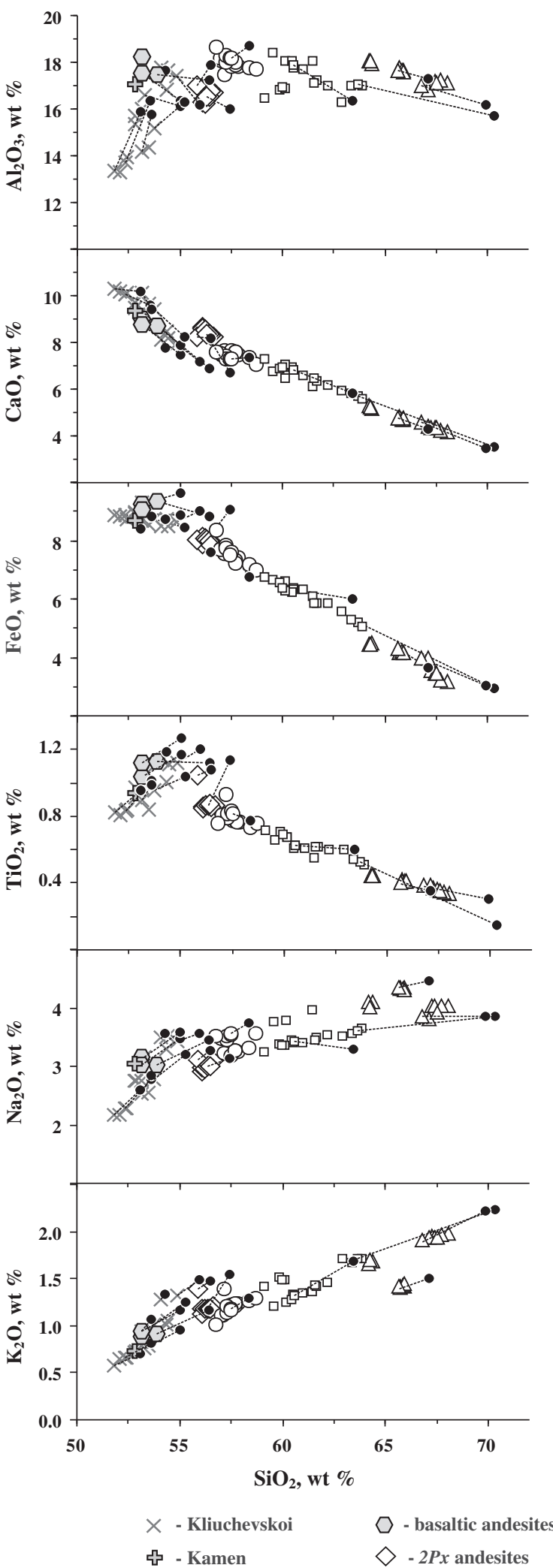

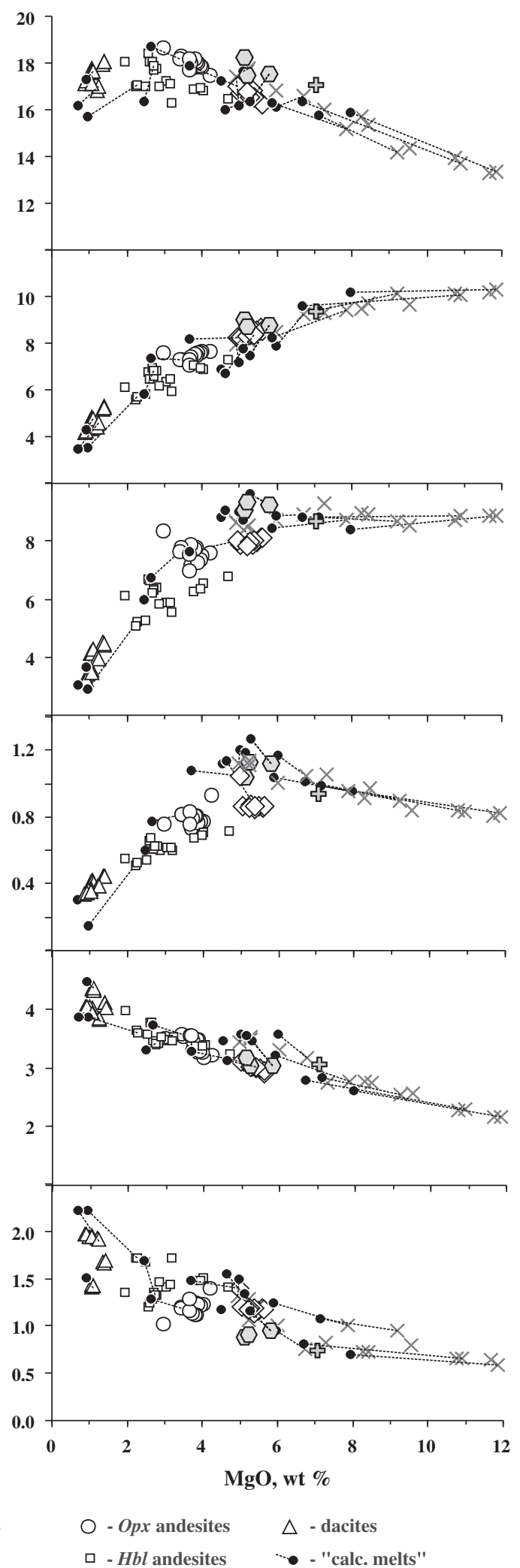

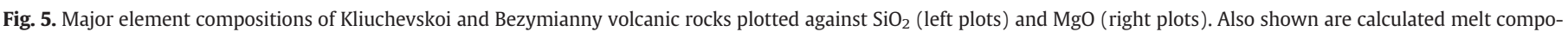
sitions obtained as a result of point-counting and modal analysis performed on representative samples (Table 1).

Kliuchevskoi and Bezymianny lavas (Fig. 7) suggests that Li was strongly partitioned into the melt and it is not affected by degassing processes (Herd et al., 2004). Excluding some non-systematic outliers, a number of trace element ratios such as $\mathrm{Ba} / \mathrm{La}, \mathrm{Be} / \mathrm{Ce}, \mathrm{Th} / \mathrm{U}, \mathrm{Y} / \mathrm{Yb}, \mathrm{La} / \mathrm{K}_{2} \mathrm{O}, \mathrm{Pb} / \mathrm{K}_{2} \mathrm{O}$, and $\mathrm{Nb} / \mathrm{Ce}$ remain nearly constant over the whole Li range thus indicating a similar mantle source or the same source variability for basalts, andesites, and dacites. It is noteworthy that both Bezymianny and preBezymianny lavas inherit trace element ratios from the Kliuchevskoi 

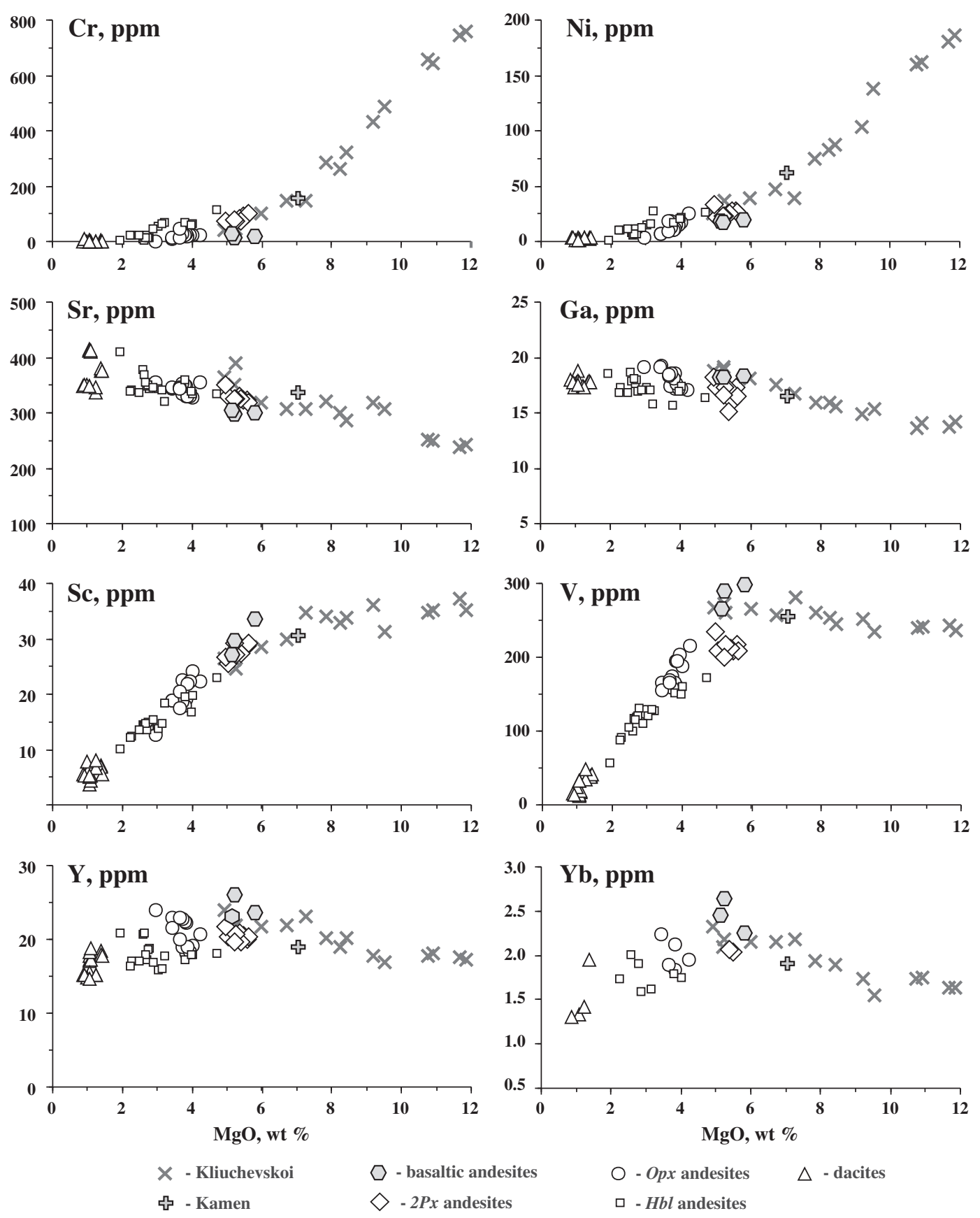

$$
\bigcirc \text { - } O p x \text { andesites } \triangle \text { - dacites }
$$

Fig. 6. Compatible trace element compositions of Kliuchevskoi and Bezymianny volcanic rocks.

less-differentiated lavas and collectively may represent the same array as was proposed for the mantle-derived CKD parental magmas (Tolbachik and prehistoric (4.6 ppm Li) and historic (6.4 ppm Li) Kliuchevskoi Volcano compositions (Portnyagin et al., 2007a) shown by black and open thick crosses respectively in Fig. 11).

Large ranges of trace element ratios are observed for $\mathrm{Hf} / \mathrm{Nb}, \mathrm{Nb} / \mathrm{K}_{2} \mathrm{O}$, and $\mathrm{Ba} / \mathrm{Rb}$. $\mathrm{Hf} / \mathrm{Nb}$ (and $\mathrm{Zr} / \mathrm{Nb}$, not shown since $\mathrm{Hf} / \mathrm{Zr}$ is nearly constant) are decoupled from each other. $\mathrm{Hf} / \mathrm{Nb}$ tend to be lower in dacitic compositions of the pre-Bezymianny stage suggesting the importance of zircon crystallization in evolved lavas during late crystallization stages (see Section 5.2 below).

$\mathrm{Nb} / \mathrm{K}_{2} \mathrm{O}$ demonstrates the highest scatter even for primitive compositions, an observation which strongly supports ideas about mantle compositional variability beneath Kliuchevskoi with respect to some
HFSE (Nb, Ta) (Portnyagin et al., 2007a, 2007b). On the Nb/ $\mathrm{K}_{2} \mathrm{O}$ versus Li plot, pre-Bezymianny stage lavas differ from Bezymianny stage lavas; they exhibit two different trends of $\mathrm{Nb} / \mathrm{K}_{2} \mathrm{O}$ decrease at given Li. In addition, old dacites from different lava domes have contrasting $\mathrm{Nb} / \mathrm{K}_{2} \mathrm{O}$, indicating strong $\mathrm{Nb}$ (and Ta) source heterogeneity.

Ba correlates linearly with $\mathrm{K}_{2} \mathrm{O}$, whereas $\mathrm{Rb}$ exhibits slightly different slopes in Kliuchevskoi and Bezymianny rocks (Fig. 7). However, the $\mathrm{Ba} / \mathrm{Rb}$ ratio is quite distinct for different lavas: only a few andesites, basaltic andesite, and basalt from Kamen Volcano have Ba/Rb similar to that of Kliuchevskoi. Most pre-Bezymianny lavas and a few andesites from stages $\mathrm{B}-\mathrm{I}$ to $\mathrm{B}$-III have systematically lower $\mathrm{Ba} / \mathrm{Rb}$ ratios, and the $\mathrm{Ba} / \mathrm{Rb}$ decrease correlates with the degree of differentiation (e.g. $\mathrm{K}_{2} \mathrm{O}$, Fig. 7). Due to the lack of $\mathrm{Rb}$ analyses for primitive melts in Portnyagin et al. (2007a) we cannot evaluate the range of $\mathrm{Ba} / \mathrm{Rb}$ in the mantle- 

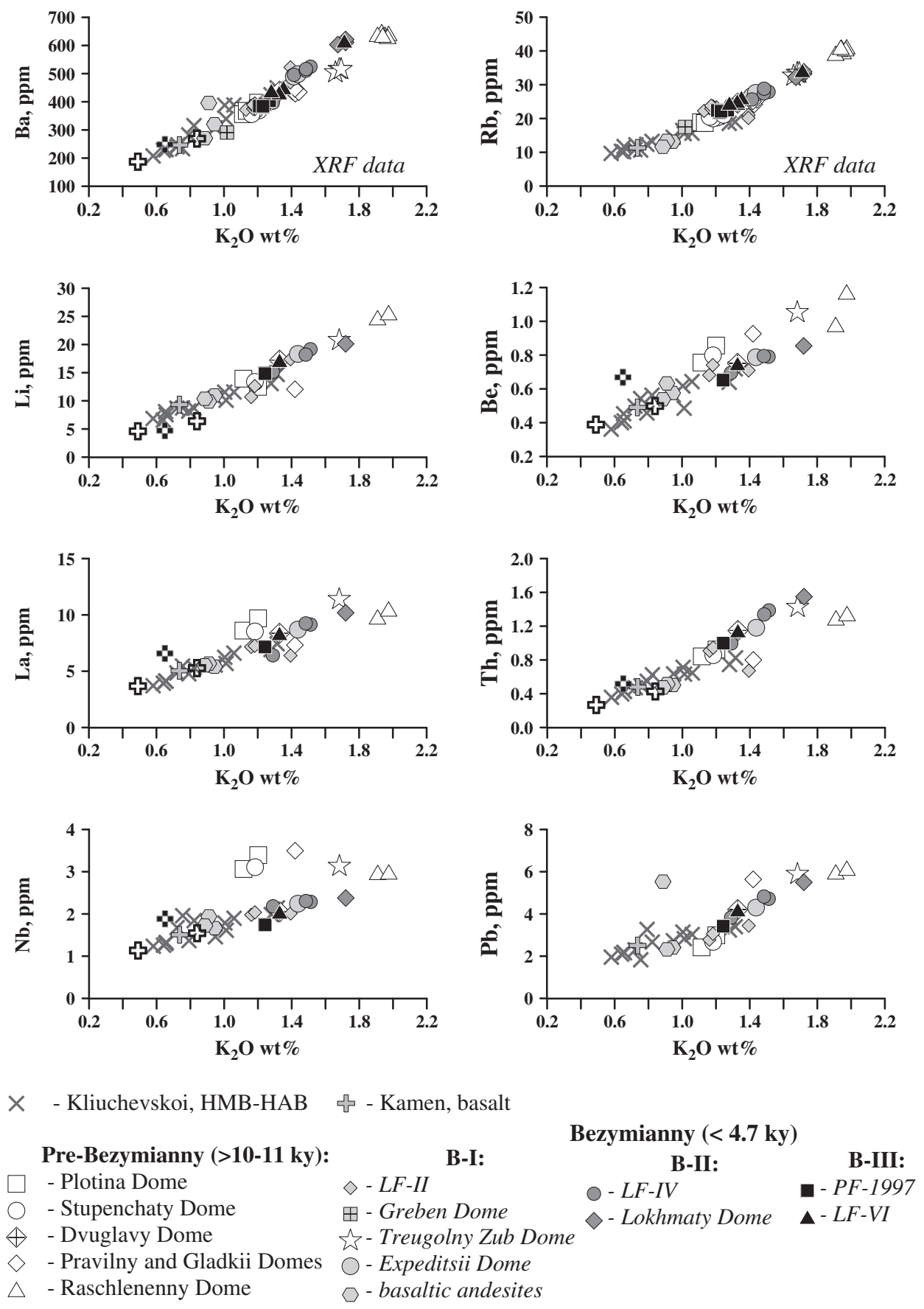

CKD primitive parental melts (Portnyagin et al., 2007):

$$
\text { њ-Kliuchevskoi } \$ \text {-Tolbachik }
$$

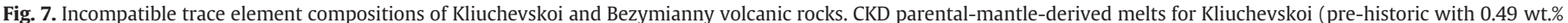
$\mathrm{K}_{2} \mathrm{O}$ and historic with 0.84 wt.\% $\mathrm{K}_{2} \mathrm{O}$ ) and Tolbachik volcanoes are from Portnyagin et al. (2007b).

derived melts; however, this ratio seems not to change significantly in less-differentiated Kliuchevskoi basalts. We think that the source mantle could be heterogeneous in $\mathrm{Ba} / \mathrm{Rb}$, which would explain the large variations of these elements.

Despite a little scatter observed for many samples, one sample (OB-40, open diamond in Figs. 7 and 11) is systematically off the common trend on many plots. This sample from Pravilny lava dome exhibits slightly higher $\mathrm{Nb} / \mathrm{Ce}, \mathrm{Be} / \mathrm{Ce}, \mathrm{Pb} / \mathrm{K}_{2} \mathrm{O}, \mathrm{Sr} / \mathrm{Y}$ (not shown), and $\mathrm{Zr} / \mathrm{Hf}$ (not shown), and lower $\mathrm{Y} / \mathrm{Yb}, \mathrm{Th} / \mathrm{U}, \mathrm{Ce} / \mathrm{Pb}$ (not shown), and $\mathrm{Li} / \mathrm{Be}$ (not shown) trace element ratios. It is also slightly lower in $\mathrm{K}_{2} \mathrm{O}$ and $\mathrm{Li}$ and higher in $\mathrm{FeO} / \mathrm{MgO}$ than other dacites (Fig. 4b, c) This suggests that the Pravilny lava dome mantle source characteristics (and probably 

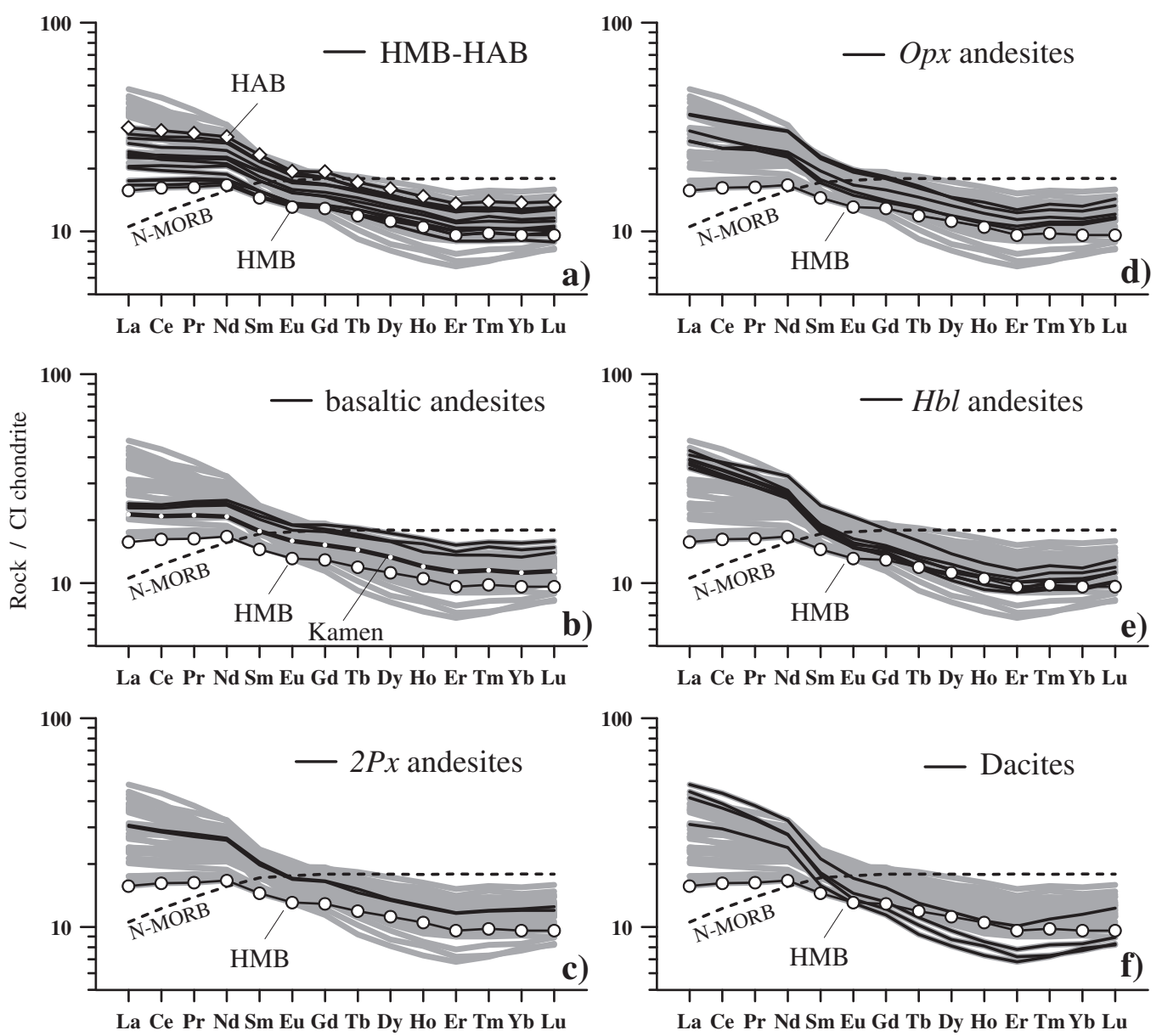

Kliuchevskoi-Bezymianny (all samples)

Fig. 8. REE spectra in Kliuchevskoi and Bezymianny volcanic rocks.

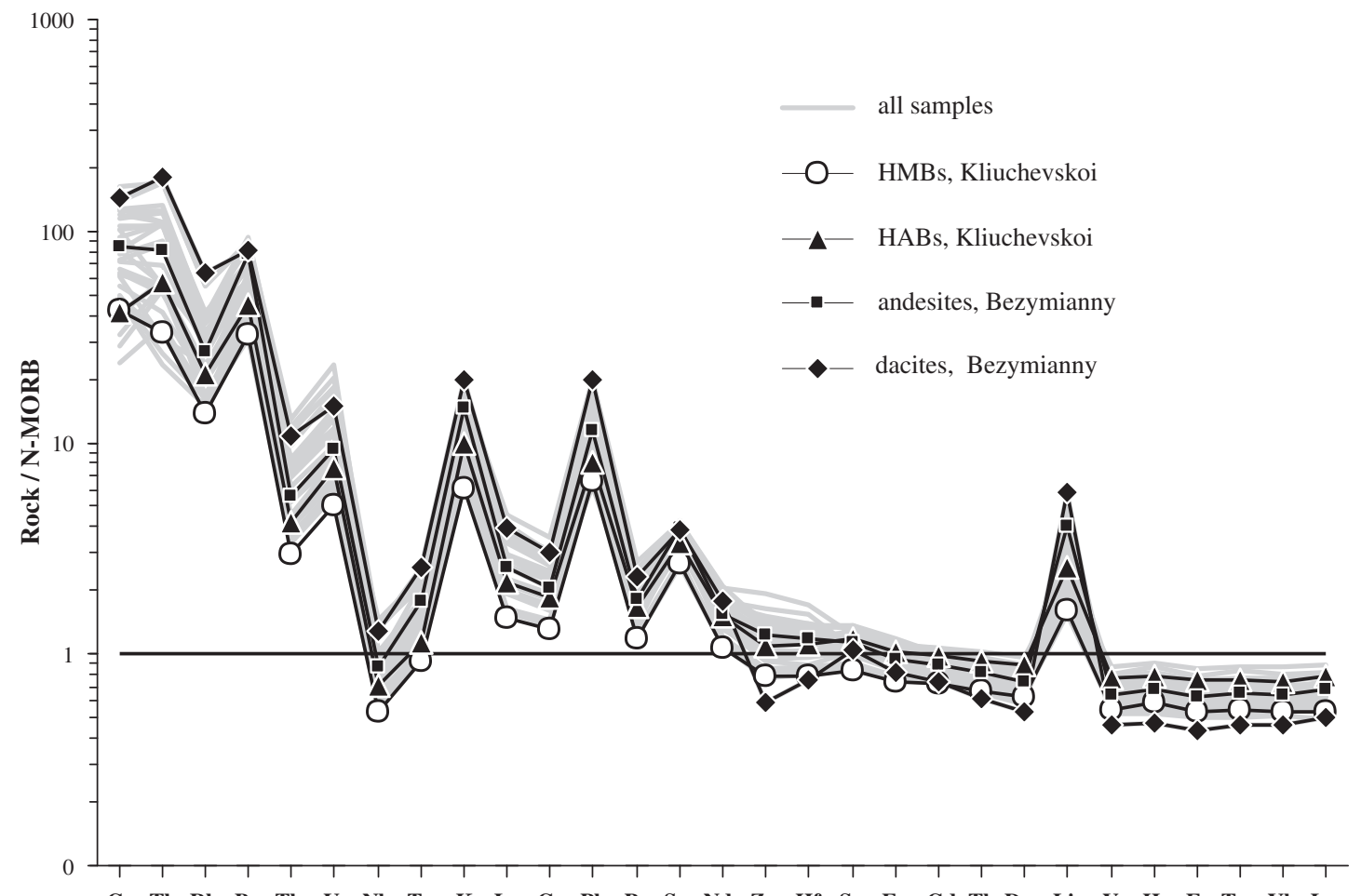

Fig. 9. Trace element patterns in selected Kliuchevskoi and Bezymianny volcanic rocks. 

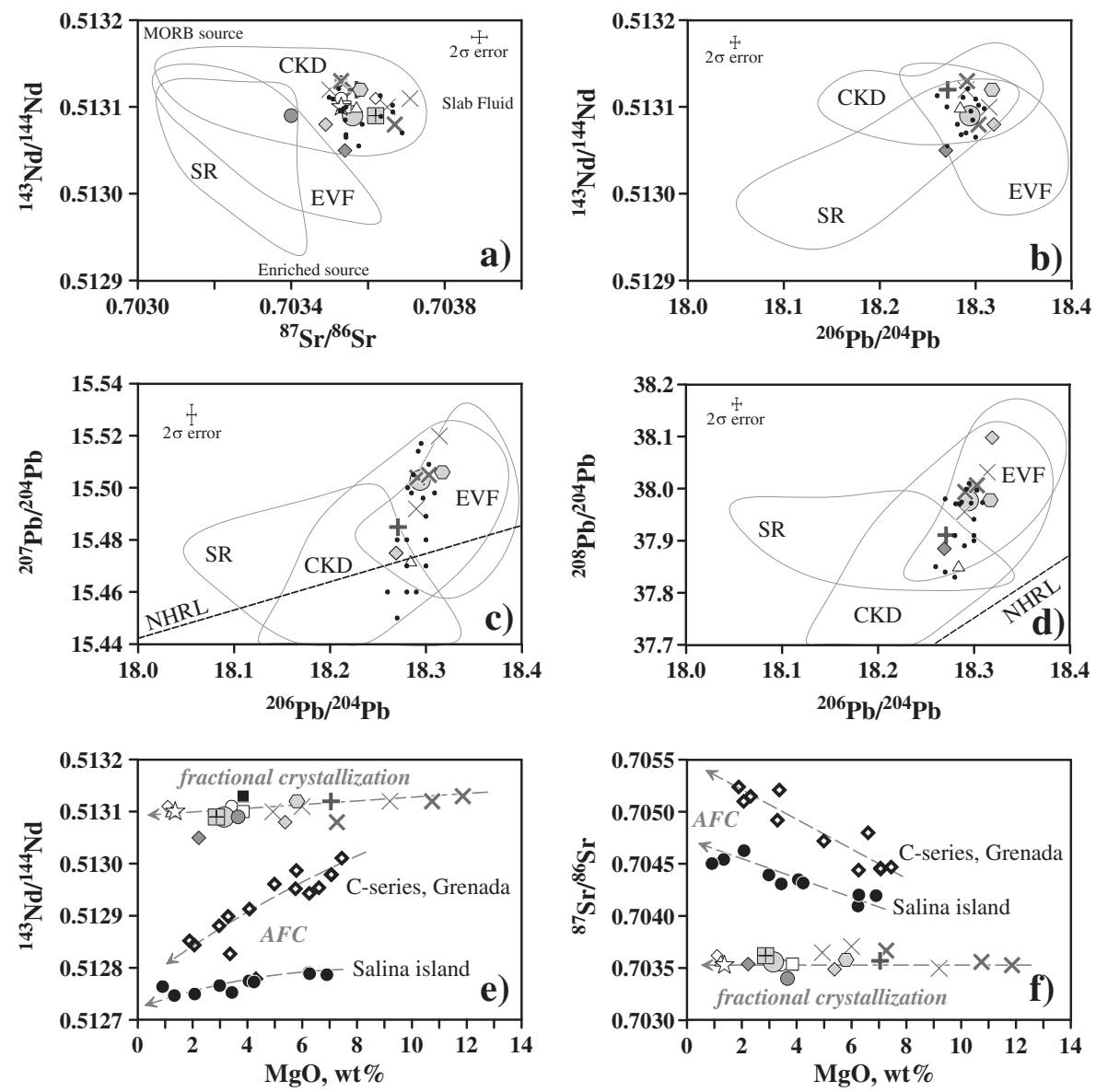

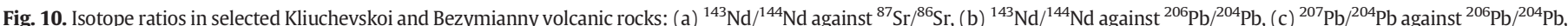

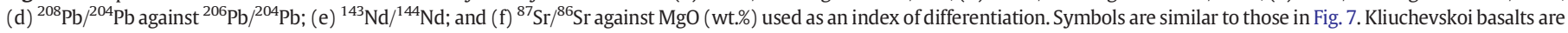

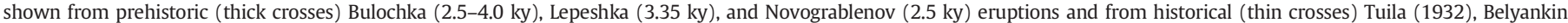

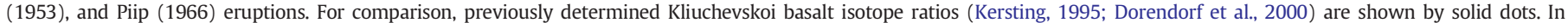

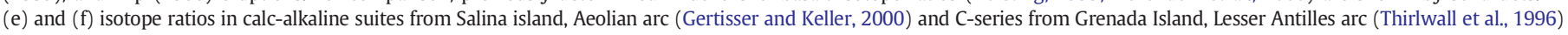

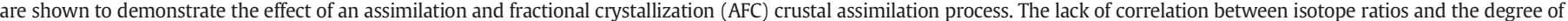
rock differentiation indicates that crystal-liquid fractionation is the dominant process in the genesis of Kliuchevskoi and Bezymianny magmas.

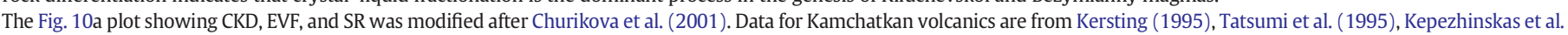
(1997), Ozerov et al. (1997), Turner et al. (1998), Dorendorf et al. (2000), and Churikova et al. (2001).

those of the Gladkii lava dome which exhibits identical major element composition and is spatially and temporally very close to the Pravilny) were probably quite distinct.

Although there are several features of incompatible elements arguing for source mantle heterogeneity, most of the chemical peculiarities in the Kliuchevskoi-Bezymianny system are best explained by magma differentiation, such as fractional crystallization or mixing. The relative role of these processes will be examined in the following sections.

\subsection{Liquid lines of descent: the combined effect of crystal fractionation} and magma mixing?

The striking feature of the Kliuchevskoi and Bezymianny lavas is the linearity of their geochemical trends with a common inflection point at $\mathrm{MgO}=\sim 6.0 \mathrm{wt} . \%$ observed for both major and compatible trace elements. Whole rock compositions are not always realistic proxies of liquid lines of descent (LLDs) since crystal accumulation may wipe out true cotectic mineral proportions (Eichelberger et al., 2006). In fact, there is evidence of disequilibrium conditions during crystallization as shown, for example, by complex phenocryst zoning, sieved Plag textures, and bimodal Plag composition in the Kliuchevskoi-Bezymianny system.
However, strong linear trends with inflection points, the lack of significant scatter for most major and trace elements, and the relatively low phenocryst mode (5-25 vol.\%, Table 1 ), suggest that the Kliuchevskoi-Bezymianny geochemical trends can be approximated as LLDs. Calculated groundmass (= liquid) compositions are apparently more silica rich, but they are generally located on the trends defined by the whole-rock compositions (see calculated melt composition in Fig. 5). Only in basaltic andesite and $2 P x$ andesite there is a discrepancy between bulk-rock and groundmass compositions with respect to $\mathrm{FeO}$, $\mathrm{TiO}_{2}$, and $\mathrm{CaO}$.

In contrast to whole rocks, calculated liquids have fairly constant or slightly increased $\mathrm{FeO}$ and $\mathrm{TiO}_{2}$, indicating the low crystallization proportion or still insignificant presence of $M t$ in the crystallizing mineral assemblage (Fig. 5). We observed the same behavior in our crystallization experiments performed on Bezymianny basaltic andesite compositions at $100 \mathrm{MPa}$ (Almeev et al., 2012): the experimental melt compositions in equilibrium with $\mathrm{Pl}+\mathrm{Cpx}+\mathrm{Opx}+\mathrm{Mt}$ were also slightly enriched in $\mathrm{TiO}_{2}$. These observations contradict the prominent $\mathrm{V}$ and $\mathrm{TiO}_{2}$ depletion observed in andesites and dacites (Fig. 6). Due to $\mathrm{V}$ and Ti partitioning in Fe-Ti oxides (as indicated by the partition coefficient of $\mathrm{V}$ and Ti between Mt and groundmass in andesite $>10$ (Luhr and Carmichael, 1980)) $\mathrm{V}$ and $\mathrm{TiO}_{2}$ depletions can be attributed to $\mathrm{Mt}$ (or Ilm) precipitation. 

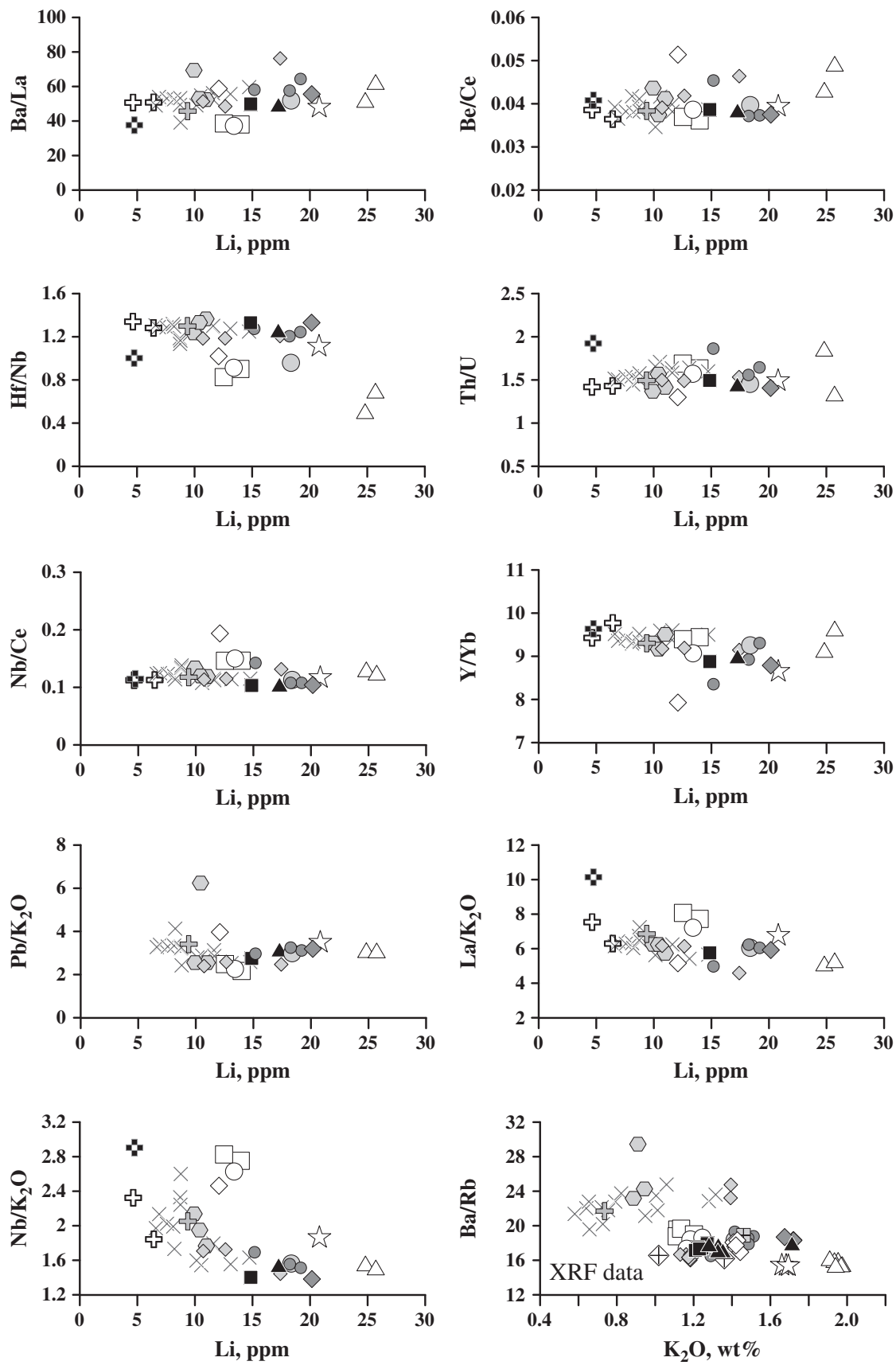

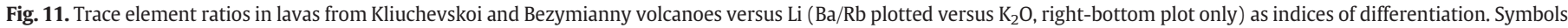
are similar to those in Fig. 7. Note that composition of the Pravilny lava dome (open diamonds) exhibits slightly different ratios of many trace elements (also see text). CKD parental-mantle-derived melts (for Kliuchevskoi and Tolbachik) are from Portnyagin et al. (2007b).

We observe Ti depletion in bulk-rock compositions but weak Ti enrichment in calculated/experimental melts (Fig. 5) because the bulk-rock compositions of $2 P x$ andesites may also be the product of magma mixing, and their original LLDs are hidden by this additional process. The importance of magma mixing in the genesis of Bezymianny lavas is illustrated in Fig. 12. Together with Kliuchevskoi basaltic lavas, the intermediate and evolved pre-Bezymianny stage rocks demonstrate a prominent crystal fractionation trend. For example on the $\mathrm{Ni}-\mathrm{Rb}$ plot there is a strong decrease in $\mathrm{Ni}$ in a very narrow range $(5-10 \mathrm{ppm})$ of $\mathrm{Rb}$, followed by nearly constant $\mathrm{Ni}(<10 \mathrm{ppm})$ across a wide range (10-40 ppm) of $\mathrm{Rb}$ concentrations (all pre-Bezymianny stage lavas are open symbols in Fig. 12a). The beginning of $\mathrm{Zr}$ crystallization in pre-Bezymianny melts is also recorded by the sharp drop of $\mathrm{Zr}$ in andesite-dacite compositions at $\sim 25 \mathrm{Rb}$ ppm (Fig. 12b).
In contrast, all younger Bezymianny lavas (Stages B-I to B-III) plot along mixing lines that connect less-differentiated basaltic andesite compositions with the most evolved dacites (Fig. 12). Major elements provide further evidence for the contribution of magma mixing in the generation of young Bezymianny rocks. For example, pre-Bezymianny lavas are systematically higher in $\mathrm{Na}_{2} \mathrm{O}$; they exhibit a convex trend which can be attributed to an increased proportion of sodic Plag crystallization in andesites and dacites. In contrast, younger lavas are lower in $\mathrm{Na}_{2} \mathrm{O}$ at the same $\mathrm{SiO}_{2}$ and plot along mixing line(s) between basaltic andesite and dacite end-members. The $\mathrm{Na}_{2} \mathrm{O}$ concentrations of $2 \mathrm{Px}$ andesites tend to be even lower, indicating (1) mixing with more primitive basaltic end-members, or (2) a higher proportion of Ca-rich Plag, probably resulting from degassing-driven crystallization (see below). $\mathrm{P}_{2} \mathrm{O}_{5}$ variations indicate that apatite crystallization occurred in the 

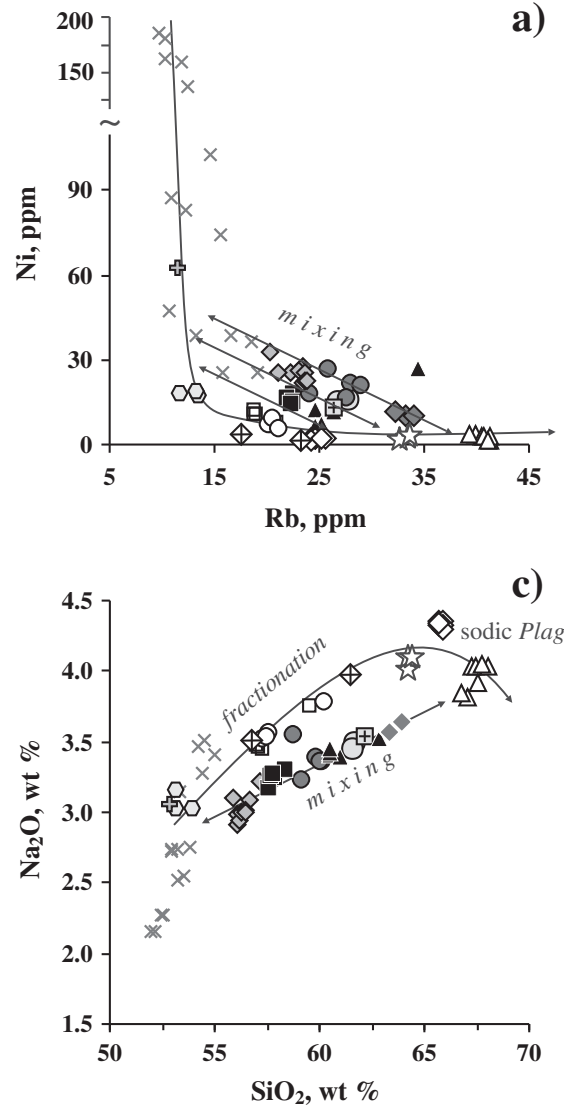

$\times$ HMBs-HABs, Kliuchevskoi

pre-Bezymianny (>10-11 ky)

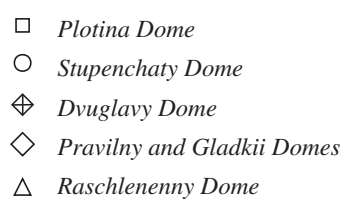

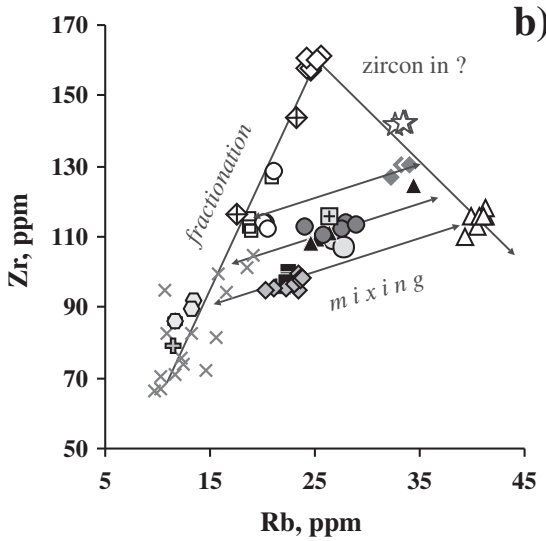

b)

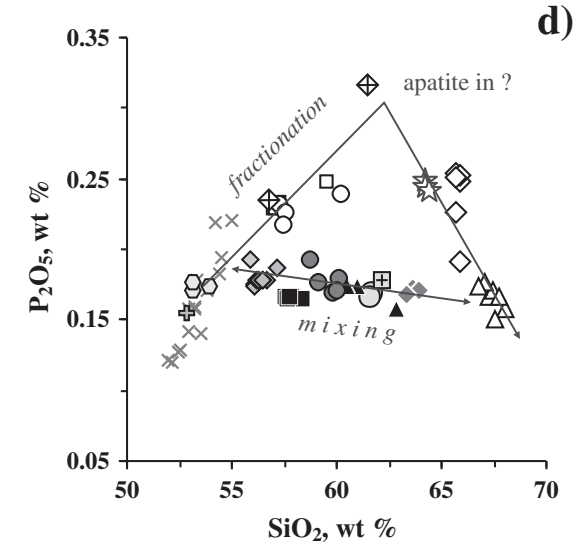

\& basalt, Kamen

Bezymianny $(<4.7 \mathrm{ky})$

\begin{tabular}{|c|c|c|}
\hline B-I & B-II & B-III \\
\hline$\diamond \quad L F-I I$ & $\bigcirc L F-I V$ & 口 $P F-1997$ \\
\hline Greben Dome & $\diamond$ Lokhmaty Dome & $\boldsymbol{\Delta} L F-V I$ \\
\hline ¿ Treugolny Zub Dome & & \\
\hline$\bigcirc$ Expedition Dome & & \\
\hline$\square$ basaltic andesites & & \\
\hline
\end{tabular}

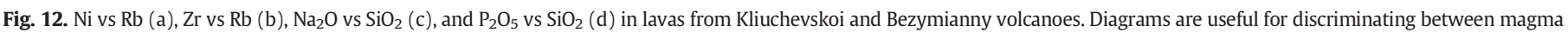
fractionation and magma mixing processes which may control compositional variations during the pre-Bezymianny and Bezymianny stages of volcano evolution (see also text).

pre-Bezymianny magmas with $\mathrm{SiO}_{2}=\sim 63$ wt.\% (approximately corresponding to $\mathrm{Rb}=\sim 25$ ppm; Fig. 12d).

All these observations illustrate the importance of basic magma replenishment to the differentiated (probably stratified) magma chamber. At the same time, mixing of $\mathrm{V}$ - and $\mathrm{TiO}_{2}$-deficient dacite melt with $\mathrm{V}$ - and $\mathrm{TiO}_{2}$-rich basaltic andesite melt will drive andesite composition to be less enriched in these elements in bulk rocks but not in the calculated melt compositions.

Here we come to a preliminary conclusion that magma mixing should be considered to be an important factor in the origin of Bezymianny andesites, especially their trace element systematics. Magma mixing signatures can also be found for major elements, although alternative mechanisms are also possible. Therefore, below, we continue examining whole rock major element compositions as a proxy of LLDs, keeping the effects of magma mixing in mind.

\subsection{Whole rock chemical variations in mineral pseudo-ternary space}

We use the approach of Grove and Kinzler (1986) by recasting lava compositions into mineral components and projecting those components onto pseudo-ternary diagrams (Grove, 1993) (Fig. 13). This allows us to trace crystallization paths of natural magmas/melts along different mineral cotectics (Sisson and Grove, 1993; Grove et al., 2003) assuming that the trends defined by the bulk-rock composition represent the LLDs.

It should be noted that the positions of mineral saturation and reaction boundaries depends on magma composition and pressure (Tormey et al., 1987; Sisson and Grove, 1993; Yang et al., 1996; Grove et al., 2003) and water activity (Grove et al., 1982; Botcharnikov et al., 2008; Almeev et al., 2012). Therefore, we can only estimate crystallization conditions for natural lavas, in particular hydrous island arc lavas, if cotectics are derived by experiments with compositions similar to that of their natural counterparts. Otherwise the saturation and reaction boundaries can only be utilized as a reference of certain thermodynamic conditions.

For the Kliuchevskoi-Bezymianny basalts, andesites, and dacites, these ternary projections are useful to demonstrate the general trend of magma evolution driven by precipitation of different mineral assemblages. For example, the Kliuchevskoi lavas demonstrate strong $\mathrm{Al}_{2} \mathrm{O}_{3}$ enrichment (Fig. 5). The evolutionary path is directed from the $\mathrm{Ol}+\mathrm{Cpx}$ sideline towards the Plag apex until melt reaches HAB and basaltic andesite compositions (Fig. 13a, b, c). These alumina-rich 

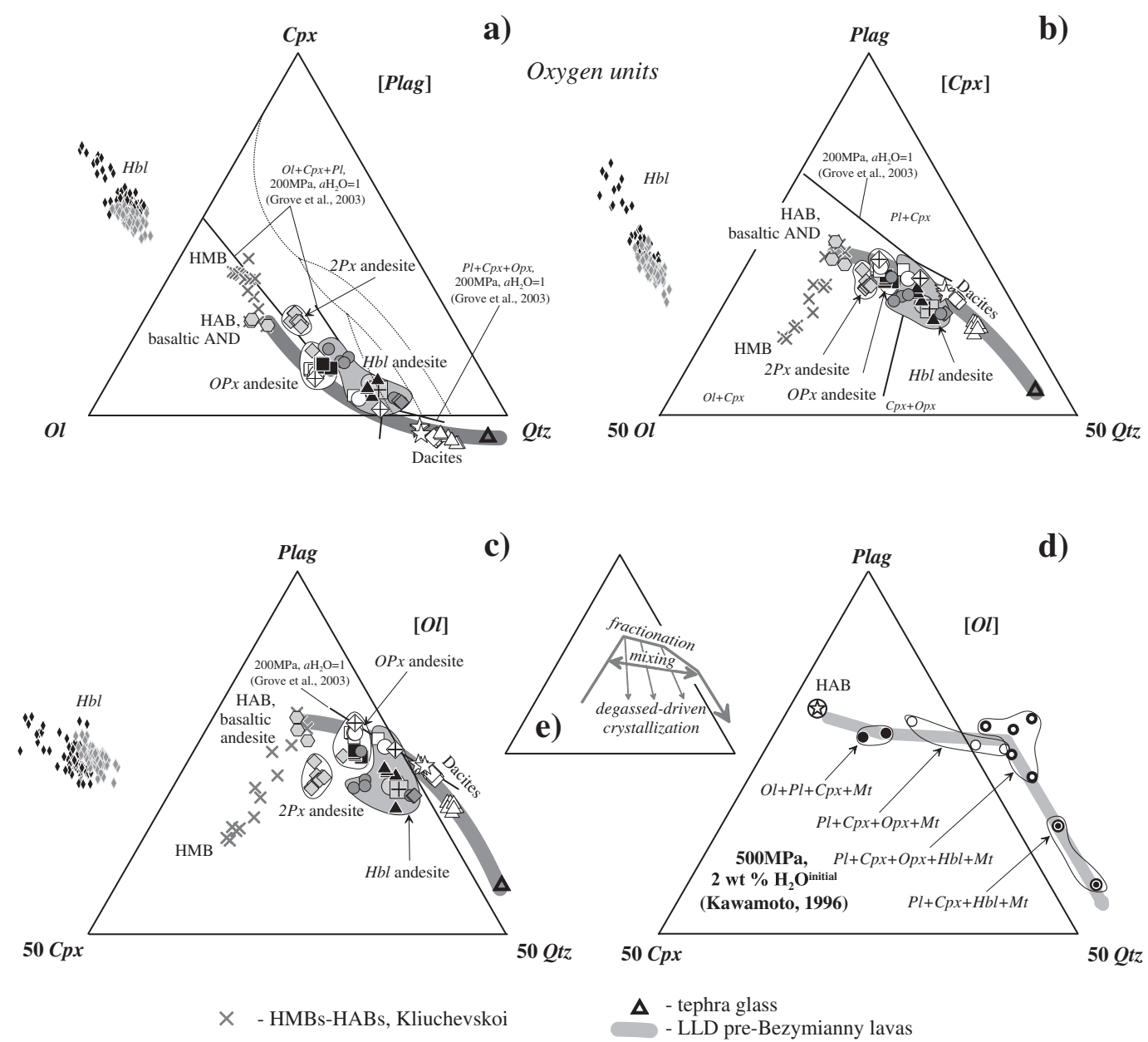

d)
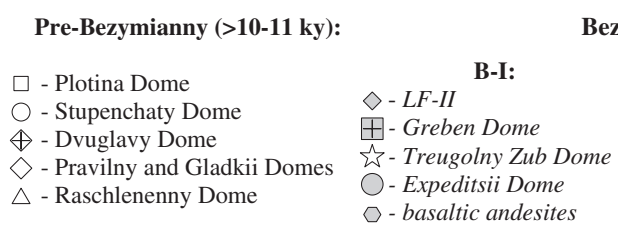

- $\mathrm{Hbl}$ from basaltic andesite OB-21

- $H b l$ from dacite OB-40

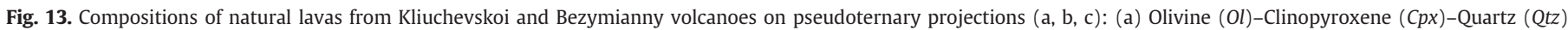

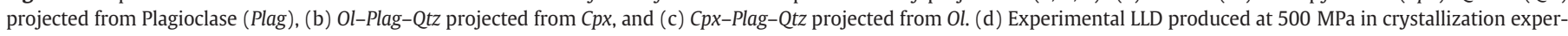

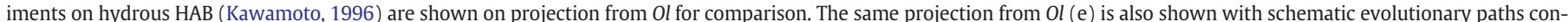

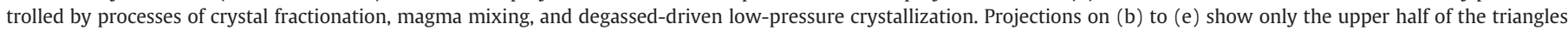

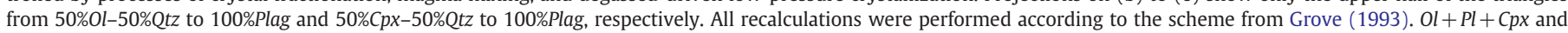

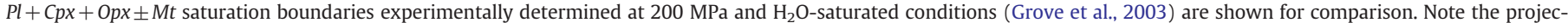
tions of natural $\mathrm{Hbl}$ compositions outside of the ternary plots.

melts represent the final stages of decompressional fractional crystallization of the initial HMB (Ariskin et al., 1995; Ariskin, 1999). At this stage Plag starts to crystallize, driving melts away from the Plag apex towards silica-rich compositions. Finally, melts reach rhyolite compositions with $73.8 \% \mathrm{SiO}_{2}$ and $0.53 \% \mathrm{MgO}$ as recorded by Bezymianny tephra glasses (in Fig. 13 a thick open triangle represents the average of 45 composition analyses; M. Portnyagin, pers. comm.).

The Bezymianny lavas are scattered between Kliuchevskoi HAB and the silica-rich tephra glass compositions and demonstrate two important features (Fig. 13a, b, c). First, different groups of $2 P x$ andesite, $\mathrm{Opx}$ andesite, $\mathrm{Hbl}$ andesite, and dacite define restricted fields on all the pseudo-ternary plots (Fig. 13) demonstrating that the change in melt compositions occurs when the mineral assemblage is changed. This suggests that lava compositions may closely resemble magmatic liquids, which inherit their compositional variations caused by fractional crystallization (controlled by crystal-melt equilibria). Systematic evolution of the experimental melts along different sets of mineral cotectics has been investigated in detail by Kawamoto (1996) who performed his experiments on $\mathrm{HAB}$ with 2 wt.\% initial $\mathrm{H}_{2} \mathrm{O}$ at $500 \mathrm{MPa}$ and showed liquids in equilibrium with different mineral assemblages (Fig. 13d). Crystallizing liquids continuously evolve along the following set of saturation and reaction boundaries: $\mathrm{Ol}^{\mathrm{OUT}}+\mathrm{Plag}+\mathrm{Cpx}+$ $\mathrm{Mt} \rightarrow \mathrm{Plag}+\mathrm{Cpx}+\mathrm{Opx}^{I N}+\mathrm{Mt} \rightarrow \mathrm{Plag}+\mathrm{Cpx}+\mathrm{Opx} \mathrm{OUT}^{\mathrm{UT}} \mathrm{Hbl}^{I N}+\mathrm{Mt} \rightarrow$ $\mathrm{Plag}+\mathrm{Cpx}+\mathrm{Hbl}+\mathrm{Mt}$, where $I N$ and OUT in superscript indicate phases which appear (IN) and disappear (OUT) from the liquidus phase. Such mineral assemblages are commonly observed in natural island arc lavas (e.g. see figures on pages 170-171 in Wilson, 1989).

Due to the difference in starting composition, the data determined by Kawamoto (1996) cannot be compared directly with the Bezymianny LLDs. However the experimental results may serve as a proxy of 
magmatic liquid evolution. The evolutionary paths of Bezymianny lavas closely resemble experimental trend determined by Kawamoto (1996) (Fig. 13d). However, the Bezymianny melt evolution is controlled by slightly different mineral cotectics: $\mathrm{Ol}^{\mathrm{OUT}}+\mathrm{Plag}+\mathrm{Cpx}+\mathrm{Opx} \pm \mathrm{Mt}$ (transition from basaltic andesite to $2 P x$ andesite), $P l a g+C p x^{O U T}+O p x+M t$ (2Px andesite to Opx andesite), Plag $+O p x^{O U T}+H b l^{I N}+M t$ (Opx andesite to $\mathrm{Hbl}$ andesite), and Plag $+\mathrm{Hbl}+\mathrm{Mt}$ ( $\mathrm{Hbl}$ andesite to dacite). When Plag is stabilized on the liquidus of a basaltic andesite melt, its progressive crystallization with $\mathrm{Ol}$ and $\mathrm{Cpx}$ drives the liquid composition away from the Plag-Cpx or Plag-Ol sidelines towards the Qtz apex on all pseudo-ternary projections (Fig. 13). In the course of crystallization, $\mathrm{Ol}$ is consumed first and later Cpx through reaction with the melt. Neither mineral is present in $O p x$ andesite melts.

When $\mathrm{Hbl}$ starts to crystallize (note the field of natural $\mathrm{Hbl}$ outside the ternary plot), the trend of liquid evolution also changes slightly, indicating strong silica enrichment (Fig. 13). This trend is controlled by both Plag and $\mathrm{Hbl}$ components so that the melt composition follows the line connecting the Plag apex and natural $\mathrm{Hbl}$ compositions (Fig. 13a, b, c). Although the Bezymianny lavas are slightly displaced from the water-saturated cotectics at $200 \mathrm{MPa}$ and the reaction boundaries given in Sisson and Grove (1993) and Grove et al. (2003), the lavas exhibit a crystallization sequence similar to that determined by Sisson and Grove (1993), Kawamoto (1996), and Grove et al. (2003). The schematic path of the Kliuchevskoi-Bezymianny LLDs is shown in Fig. 13e by a solid polyline labeled "fractionation" (a small inset ternary diagram presents a projection from $\mathrm{Ol}$ onto the $\mathrm{Pl}-\mathrm{Cpx}-\mathrm{Qtz}$ plane).
The second observation from the pseudo-ternary diagrams is that the lavas from pre-Bezymianny and Bezymianny stages can be easily discriminated. For example, all pre-Bezymianny lavas define the LLD with the highest proportion of Plag and lowest proportion of normative $\mathrm{Qtz}$ at any given $\mathrm{Cpx}$ and $\mathrm{Ol}$ (Fig. 13b, c). In the case of Fig. 13a, the pre-Bezymianny compositions plot closer to the Plag vertex.

All Bezymianny stage lavas (<4.7 kyr) are more depleted in Plag than are pre-Bezymianny lavas. These separate trends can be interpreted as a product of mixing between dacites and basalts (see a schematic mixing line in Fig. 13e inset). 2Px andesite lavas demonstrate the most pronounced depletion in the Plag component consistent with the magma mixing hypothesis (Section 5.2). Alternatively, when an andesitic liquid undergoes Plag over-saturation due to rapid $\mathrm{H}_{2} \mathrm{O}$ loss (degassing-driven crystallization), extensive Plag crystallization occurs which drives the melt composition away from the Plag apex (see schematic dashed lines in Fig. 13e). This effect will be further discussed in a later section.

\subsection{Fractional crystallization recorded in Cpx compositions}

The importance of crystal fractionation in the genesis of KliuchevskoiBezymianny lavas is further supported by trace element variations in $C p x$ phenocrysts. The full presentation of this extensive dataset (which also includes data for Plag and $\mathrm{Hbl}$ ) requires a separate publication and will appear elsewhere. Below we discuss the most fundamental results, summarized for the trace element variations in Cpx (Fig. 14).
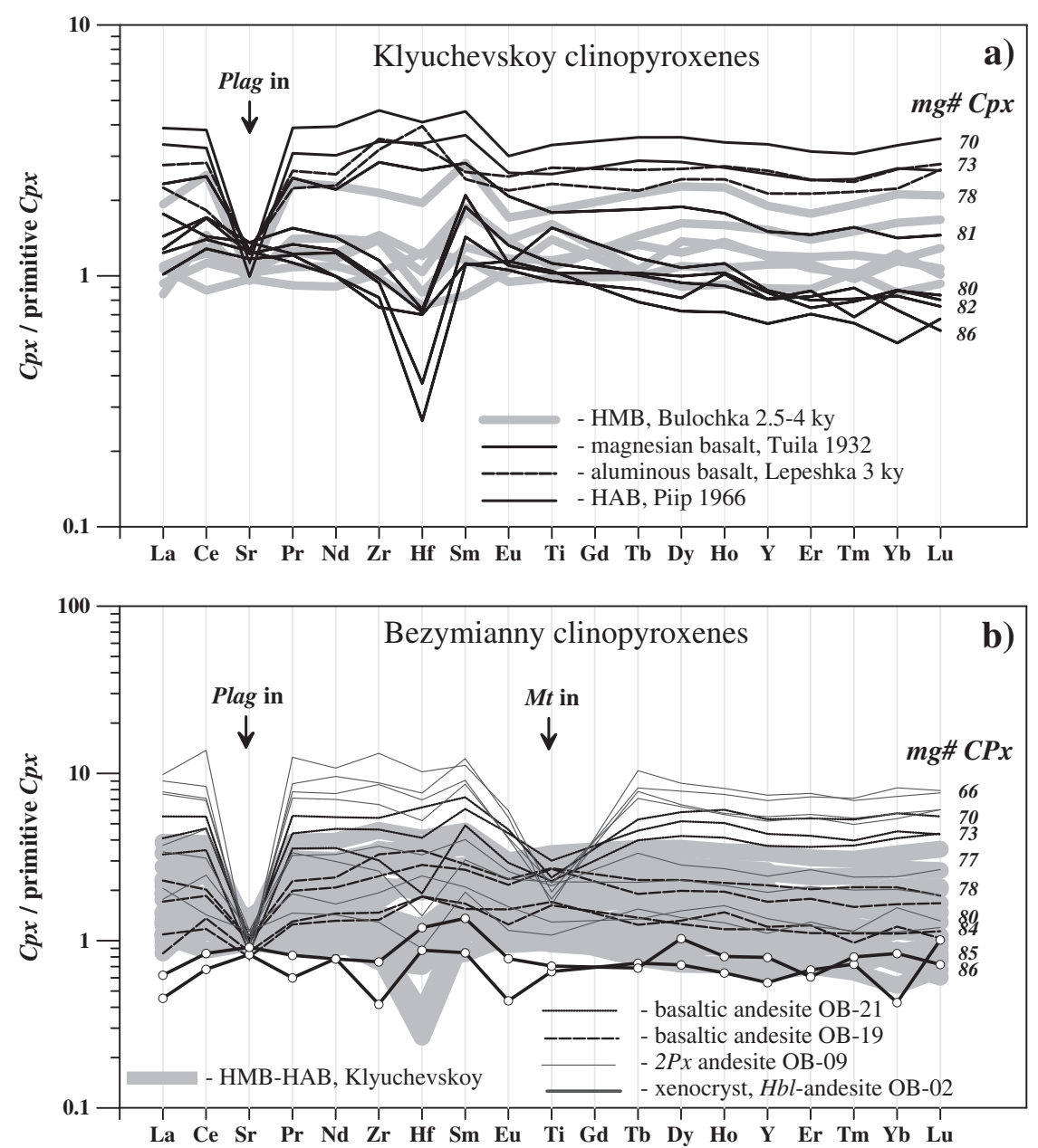

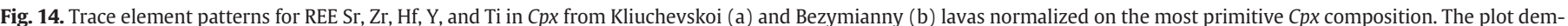

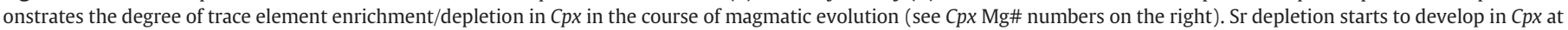
Mg\# $~ 80$ and Ti depletion develops in Cpx at Mg\# 73 with the appearance of Plag and Mt crystallization, respectively. 
Cpx trace element abundances normalized to the most primitive Cpx composition demonstrate general enrichment in incompatible trace elements with decreasing Mg\# (Fig. 14a, b). It should be noted that some Cpx from Tuila magnesian basalts and Bulochka HMB lavas have slightly different $\mathrm{Zr}$ and $\mathrm{Hf}$ abundances, inviting a discussion of source variability (Fig. 14a). This topic is beyond the scope of the present discussion and herein we simply note that variations in $D(\mathrm{Zr})$ and $D(\mathrm{Hf})$ for $C p x$-melt partitioning may reach one to two orders of magnitude (Green, 1994; Green et al., 2000).

The most important finding from $C p x$ trace element chemistry is that Bezymianny lava Cpx inherits the geochemical characteristics of Kliuchevskoi Cpx (Fig. 14b). The xenocrystic Cpx in Expeditsii dome $\mathrm{Hbl}$ andesite is more primitive than that found in Kliuchevskoi HMB lavas (Fig. 14b).

Another striking characteristics of the $\mathrm{Cpx}$ spectra are the $\mathrm{Sr}$ minimums observed in aluminous basalts, HABs, and $2 P x$ andesite, and the Ti minimums observed in basaltic andesite and 2Px andesite (Fig. 14a, b). According to the discussions above (Sections 5.2 and 5.3), elemental $\mathrm{Sr}$ and Ti depletions in $\mathrm{Cpx}$ are attributed to depletion in host melts. Therefore, onsets of Plag fractionation for $\mathrm{Sr}$ and $\mathrm{Mt}$ fractionation for Ti coincided with Cpx compositions of $\mathrm{Mg \#}_{80}$ and $\mathrm{Mg} \#_{75}$, respectively.

The magma mixing process is also recognized in the trace element Cpx composition. Cpx crystals from the same lava sometimes display different scales of $\mathrm{Sr}$ minimum; for example one $\mathrm{Cpx}$ from HMB shows a strong negative Sr spike, but $\mathrm{Cpx}$ from other HMBs do not (Fig. 14). The same is true for the Ti minimums as shown by the most magnesian Cpx from 2Px andesite, where Ti is not depleted. However other magnesian Cpx phenocrysts exhibit a strong negative Ti anomaly. These observations support the derivations of the Cpx crystals from diverse melts originated at different evolutionary stages along the similar cotectic. Therefore, magma mixing of this kind is regarded as internal mixing (automixing).

\subsection{Oxygen fugacity conditions for the Bezymianny andesites}

Compositions of coexisting Ilm-Mt pairs (e.g. Ilm inclusions in Mt phenocrysts) were used to estimate oxygen fugacity and temperatures according to the method outlined in Spencer and Lindsley (1981) with the recalculation scheme of Stormer (1983). Estimated temperature and oxygen fugacity are shown in Fig. 15. The Bezymianny lavas show a wide range from $\mathrm{Ni}-\mathrm{NiO}(\mathrm{NNO})$ to $\mathrm{NNO}+2$ with temperature ranging from 1140 to $700{ }^{\circ} \mathrm{C}$ in basalt to dacite compositions. These estimates

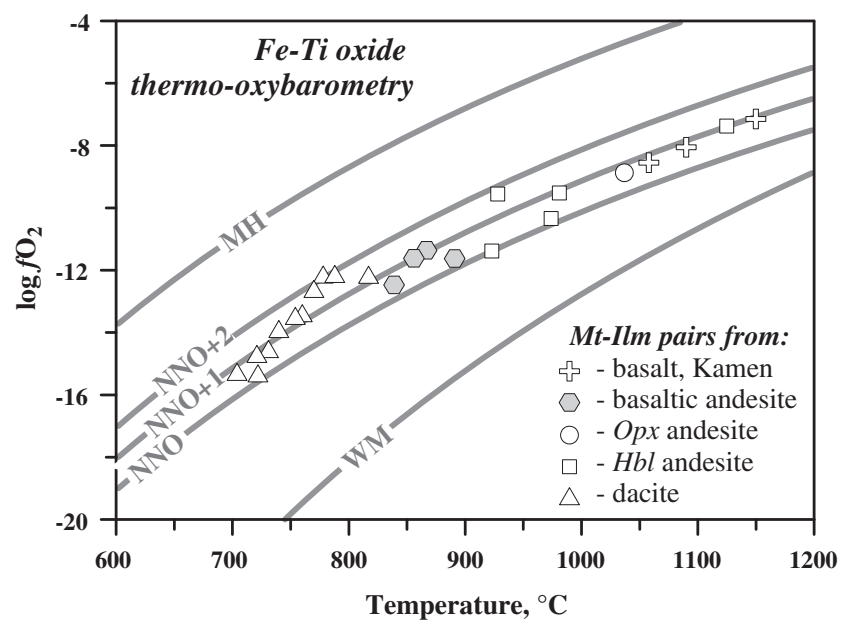

Fig. 15. Thermo-oxybarometry of Bezymianny volcanic rocks: $\log$ oxygen fugacity $\left(\log \mathrm{fO}_{2}\right)$ versus temperature, estimated from Mt-Ilm equilibria (Stormer, 1983) using the ILMAT electronic table (Lepage, 2003). The thickness of the oxygen buffer curves (NNO: Ni-NiO, MH: Mt-hematite, WM: wüstite-magnetite) corresponds to pressure variations of a particular oxygen buffer between $1 \mathrm{~atm}$ and $500 \mathrm{MPa}$. are in good agreement with known redox conditions for magmas in subduction zones (Gill, 1981; Kelley and Cottrell, 2009).

\subsection{Geothermobarometry for the Bezymianny magma plumbing system}

Temperatures and pressures calculated for representative lava compositions (Table 1) are shown in Figs. 16-17 (see Supplementary Material 5 for details of calculations). First, the amphibole geothermobarometer of Ridolfi et al. (2010) is applied for $\mathrm{Hbl}$-bearing lavas (Fig. 16). Although the uncertainty of this method utilizing only amphibole composition is larger than that of mineral-melt geothermometers (Putirka, 2008), we believe that $P-T$ variations shown in Fig. 16 are systematic for genetically related samples. Next, for each sample, $P-T$ estimates are obtained for both whole rock and groundmass compositions (symbols connected by lines in Fig. 17) assuming that whole rock-phenocryst cores and groundmassphenocryst rims do represent equilibrium assemblages (Putirka, 2008). For better comparison, the line-connected whole rock-groundmass pairs are provided for all $\mathrm{Ol}-, \mathrm{Plag}-, \mathrm{Cpx}$ - and $\mathrm{Opx}$-melt equilibria (Putirka, 2008) geothermobarometers, shown in Fig. 17 by the small inner symbols, such as circles for $\mathrm{Ol}-$, black dots for $\mathrm{Cpx}-$, crosses for Plag- and "no inner symbol" for Opx-melt equilibria.

\subsubsection{Amphibole-melt geothermobarometry for andesite-dacite}

$P-T$ conditions of crystallization determined by the amphibole geothermobarometer demonstrate strong polybaric cooling trends (Fig. 16). For example, 700-250 MPa pressures and 990-860 ${ }^{\circ} \mathrm{C}$ temperatures are obtained for Expeditsii dome andesite (OB-2), 350-200 MPa, $960-875{ }^{\circ} \mathrm{C}$ for Lokhmaty dome andesite (OB-16), and 500-400 MPa, 910-950 ${ }^{\circ} \mathrm{C}$ for Pravilny dome dacite (OB-40). It should be noted that different magmas which form discrete lava domes/lava flows define distinct $P-T$ evolutionary paths recorded in the compositions of $\mathrm{Hbl}$ phenocrysts (see regression lines for each lava dome in Fig. 16). This implies that the $\mathrm{Hbl}$-bearing andesitic to dacitic lavas may represent magma batches equilibrated at different temperatures and different depths. Most magnesian $\mathrm{Hbl}$ from less-differentiated basaltic andesites (OB-21) indicate the highest temperatures $\left(960-1000{ }^{\circ} \mathrm{C}\right)$, similar to magnesian $\mathrm{Hbl}$ from $\mathrm{Hbl-Cpx}$ cumulates hosted by Plotina dome

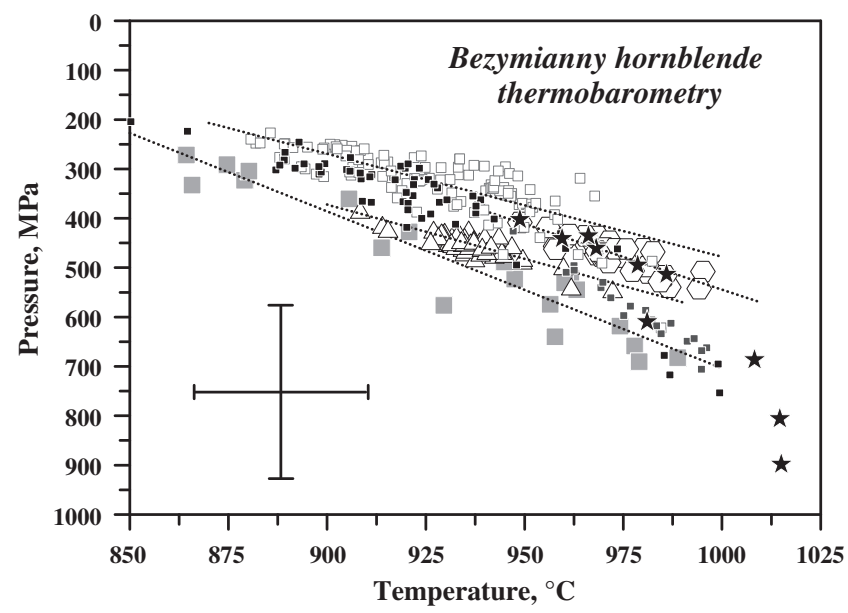

$$
\begin{array}{cc}
\text { Pre-Bezymianny: } & \text { B-II: } \\
\triangle \text { - dacite OB-40 } & -H b l \text { andesite OB-16 } \\
\star \text { - } H b l \text {-Cpx cumulates, } & \\
\text { Plotina dome } & \text { B-III: } \\
\text { B-I: } & -H b l \text { andesite, Bez97-5 } \\
\square \text { - bas. andesite OB-21 } & \\
-H b l \text { andesite OB-2 } &
\end{array}
$$

Fig. 16. Pressure-temperature estimates of $\mathrm{Hbl}$ crystallization for representative $\mathrm{Hbl}$-bearing lavas (Table 1, Fig. 4), determined by using the amphibole geothermobarometer from Ridolfi et al. (2010). See text for details. 
a) $O p x$-, $C p x$-, Plag- and $O l$-melt thermobarometry: the case of 2 and $5 \mathrm{wt} \% \mathrm{H}_{2} \mathrm{O}$ in parental melt

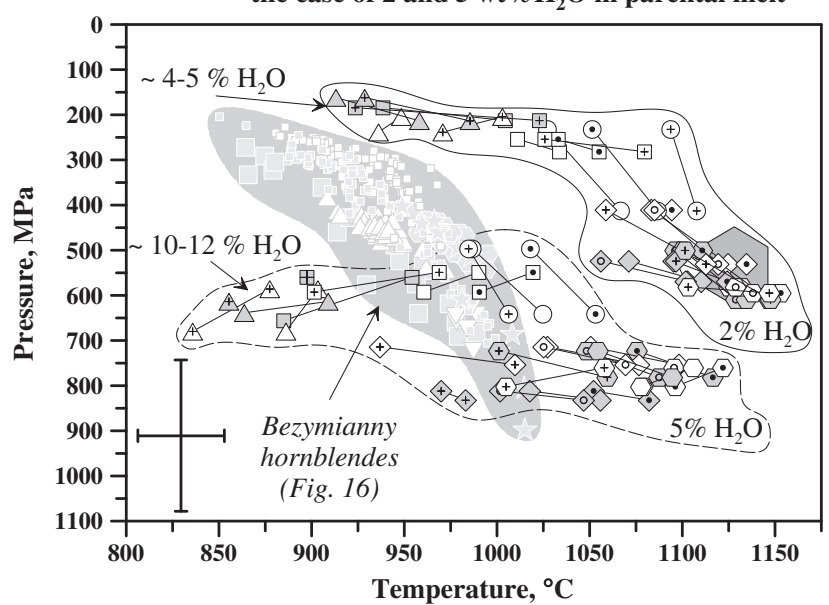

b) $O p x$-, Cpx-, Plag- and Ol-melt thermobarometry: melt $\mathrm{H}_{2} \mathrm{O}$ is chosen to produce the same temperatures using $O p x$-melt and Plag-melt equilibria $\left(T^{O p x-L}=T^{\text {Plag-L }}\right)$

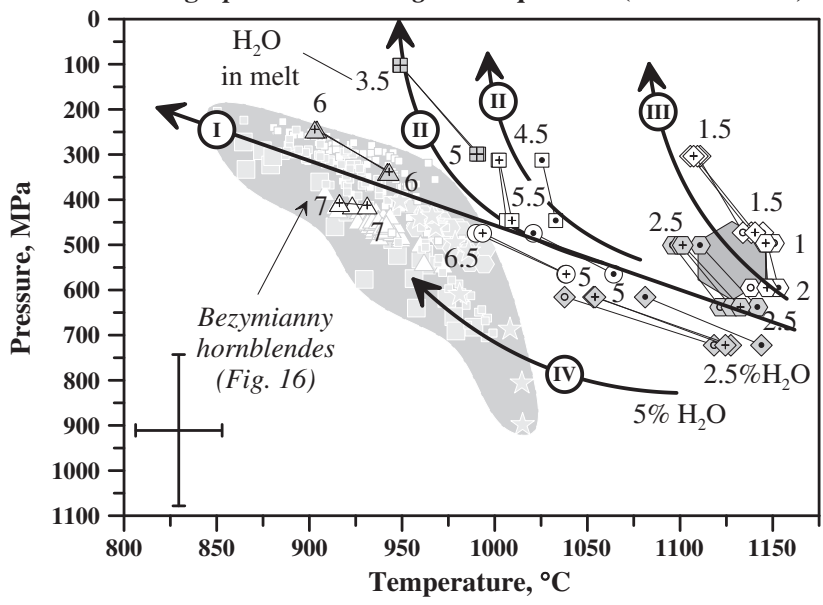

Mineral-melt (L) equilibira: $O p x-L \quad C p x-L \quad O l-L \quad P l-L$

\begin{tabular}{|c|c|c|c|}
\hline$\bullet$ & 아 & 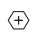 & - basaltic andesite (OB-19) \\
\hline$\bullet$ & (0) & $\oplus$ & - basaltic andesite (OB-21) \\
\hline$\diamond$ & 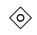 & $\stackrel{+}{ }$ & - $2 P x$ andesite (OB-9) \\
\hline 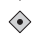 & 아 & $\leftrightarrow$ & - $2 P x$ andesite (OB-11) \\
\hline$\odot$ & & $\oplus$ & - Opx andesite (OB-42) \\
\hline$\bullet$ & & $\boxplus$ & - $\mathrm{Hbl}$ andesite (Bez97-5) \\
\hline ๑ & & $\boxplus$ & - $\mathrm{Hbl}$ andesite (OB-16) \\
\hline & & $\triangle$ & - dacite (OB-40) \\
\hline & & $\mathbb{A}$ & - dacite (OB-15) \\
\hline
\end{tabular}

$$
\begin{aligned}
& \text { - experimental } P l+C p x+O p x \text { saturation in } \\
& \text { basaltic andesite melt (Almeev et al., 2013a) } \\
& \text { - whole rock - groundmass pair }
\end{aligned}
$$

Fig. 17. Pressure-temperature $(P-T)$ estimates for representative lavas (Table 1$) . P-T$ estimates are obtained for whole rock and groundmass compositions in each sample (line-connected symbols) using $\mathrm{Opx}-, \mathrm{Cpx}-, \mathrm{Plag}-$, and $\mathrm{Ol}$-melt geothermobarometers (Putirka, 2008). (a) The case of initial 2 and 5 wt.\% $\mathrm{H}_{2} \mathrm{O}$ and Raleigh water accumulation. (b) Melt water contents were chosen to obtain the same calculated temperatures by Opxmelt and Plag-melt geothermometers. See details of calculations in the text and Supplementary Material 4. P-T conditions determined by amphibole thermobarometry (Fig. 16) are shown by the gray field. $P-T$ conditions of Plag-Cpx-Opx saturation in basaltic andesite melt experimentally determined in Almeev et al. (2012) are shown as a dark gray symbol. Note the closeness of temperatures for basaltic andesites and $2 P x$ andesites with $\sim 2 \mathrm{wt} . \%$ $\mathrm{H}_{2} \mathrm{O}$ in parental melt calculated using different thermometers and the large range of temperatures obtained with initial 5 wt.\% $\mathrm{H}_{2} \mathrm{O}$ in parental melt. See text for details. andesites. Some Expeditsii dome $\mathrm{Hbl}(\mathrm{OB}-2)$ also crystallized at high temperatures. However, the Expeditsii dome $\mathrm{Hbl}$ forms its own distinct trend in Fig. 16, showing systematically lower temperatures at given pressure. Moreover, $\mathrm{P}-\mathrm{T}$ conditions of the $\mathrm{OB}-2 \mathrm{Hbl}$ are directed towards the most magnesian $\mathrm{Hbl}$ from $\mathrm{Hbl}-\mathrm{Cpx}$ cumulates, which demonstrate the highest pressure (800-900 MPa) (Fig. 16).

Although the $P-T$ estimates based on amphibole chemistry have a high degree of uncertainty, one can follow systematic temporal changes in the $P-T$ conditions of the $\mathrm{Hbl}$-bearing magmas. For example, the oldest dacitic Pravilny dome lavas (OB-40) and Expeditsii dome $\mathrm{Hblan-}$ desites (OB-2, Stage B-I) show high calculated pressures, whereas the historical eruptions of the Stage B-II Lokhmaty dome (OB-16) and the Stage B-III 1956-eruptives crystallize $\mathrm{Hbl}$ at 100-200 MPa lower pressures (when compared at the same temperature). The $\mathrm{Hbl}$ from basaltic andesite OB-21 demonstrates lower pressures (Fig. 16). However, the age of the basaltic andesite OB-21 is not well constrained and, in general, the origin of $\mathrm{Hbl}$ in this sample is still under question (whether it is a phenocryst or xenocryst).

\subsubsection{Mineral-melt geothermobarometry for basaltic andesite}

In a previous section, amphibole geothermobarometry provided first estimates of $\mathrm{Hbl}$ crystallization $P-T$ conditions in the Bezymianny magmas. To assess $\mathrm{P}-\mathrm{T}$ conditions of both $\mathrm{Hbl}$-bearing and $\mathrm{Hbl}$-free magma storages, we applied a set of geothermobarometers from Putirka (2008). Two models with different $\mathrm{H}_{2} \mathrm{O}$ concentrations in parental and differentiated magmas were tested. In the first model we assume Raleigh fractionation with $\mathrm{H}_{2} \mathrm{O}$ accumulation (Fig. 17a). In the second model (Section 5.6.5) we assume open system fractionation, where $\mathrm{H}_{2} \mathrm{O}$ in the system was varied to obtain similar modeled temperatures by using two independent $0 p x$-melt and Plag-melt geothermometers (Fig. 17b, also see Supplementary Material 5 for details).

Thus, in our first model (Fig. 17a) with 2 wt.\% $\mathrm{H}_{2} \mathrm{O}$ in the parental melt we obtained $P-T$ conditions for the basaltic andesite and $2 P x$ andesite in a relatively narrow range using all four geothermobarometers, such as Opx-melt, Cpx-melt, Plag-melt, and Ol-melt (Putirka, 2008). Interestingly, these $P-T$ estimates are in good agreement with our experimentally determined $P-T$ conditions of $P l a g+C p x+O p x$ saturation in basaltic andesite melts (see large gray octahedrons in Fig. 17a, b; experiments are from Almeev et al., 2012). This may indicate the reliability of the thermodynamic mineral-melt pair calculations presented in this chapter. It also confirms the conclusions by Almeev et al. (2012) that the multiple saturation in the Bezymianny parental basaltic andesite magma occurs at melt $\mathrm{H}_{2} \mathrm{O}=\sim 2$ wt.\%, $\mathrm{P}=\sim 500-600 \mathrm{MPa}$, and $\mathrm{T}=$ $1100-1150{ }^{\circ} \mathrm{C}$. Thus, we define the parental magma storage depths to be $15-20 \mathrm{~km}$ beneath Bezymianny Volcano.

\subsubsection{Mineral-melt geothermobarometry of the "andesite transition" (2 wt.\% $\mathrm{H}_{2} \mathrm{O}$ initial)}

Putirka's (2008) geothermobarometers applied to intermediate melts from $\mathrm{Opx}$ andesites to $\mathrm{Hbl}$ andesites show large discrepancies between estimates for each individual sample (for both whole rock and groundmass compositions, in the case of $2 \mathrm{wt} . \% \mathrm{H}_{2} \mathrm{O}$ in parental melt). Temperatures determined by the Plag-melt geothermometer are systematically higher than those determined by $O p x$-melt or Cpx-melt geothermometers (Fig. 17a). It is obvious that to suppress Plag crystallization, modeled melts should include more $\mathrm{H}_{2} \mathrm{O}$ than those that follow from simple Raleigh fractionation.

The calculations with initial 2 wt.\% $\mathrm{H}_{2} \mathrm{O}$ also resulted in higher temperatures $\left(\sim 900-1025{ }^{\circ} \mathrm{C}\right)$ obtained by anhydrous mineral-melt geothermobarometers compared to the low temperatures ( 875$950{ }^{\circ} \mathrm{C}$ ) determined by the amphibole geothermobarometer for intermediate and silicic compositions. Although temperatures calculated by $O p x$-melt and Plag-melt geothermometers in dacite are closer, application of the geothermobarometric method to dacite composition is not as straightforward as for less differentiated magmas for two 
reasons. The first reason is that the $O p x$ is not present in dacites as phenocrysts. It exists in dacitic melts only as abundant $O p x$ inclusions in Plag phenocrysts. The second reason is related to the choice of natural Plag composition to be in equilibrium with the dacitic bulk-rock or groundmass compositions. In general, the Plag composition depends on melt composition, temperature, pressure, and water activity. The empirical parameterization of $\mathrm{KD}^{\mathrm{Na}-\mathrm{Ca}}$ between Plag and melt (Hamada and Fujii, 2007) used in our study for the selection of equilibrium Plag compositions may be problematic since dacite compositions were not included in the Hamada and Fujii (2007) calibration. Thus, the $P-T$ estimates for dacite need to be considered with caution.

5.6.4. Role of variable $\mathrm{H}_{2} \mathrm{O}$ contents on mineral-melt equilibria (5 wt.\% $\mathrm{H}_{2} \mathrm{O}$ initial)

As was mentioned above, comparison of the amphibole geothermobarometer and "anhydrous mineral"-melt geothermobarometers, assuming closed system fractionation and using 2 wt.\% $\mathrm{H}_{2} \mathrm{O}$ in parental basaltic andesite, resulted in a $P$-T estimate discrepancy for differentiated $\mathrm{Hbl}$-bearing andesite, which almost certainly crystallized under more hydrous conditions. It is also evident from Fig. 17a that in the case of 2 wt.\% initial $\mathrm{H}_{2} \mathrm{O}$, "high pressure" amphiboles from the $\mathrm{Hbl}-\mathrm{Cpx}$ cumulates cannot be linked to their parental basaltic andesite magmas through polybaric or isobaric crystallization processes.

We examined $5 \mathrm{wt} . \% \mathrm{H}_{2} \mathrm{O}$ in parental magmas to test the possibility of crystallization of "high pressure" $\mathrm{Hbls}$. The $P-T$ estimates reasonably shifted to lower temperatures and higher pressures for all melt compositions (Fig. 17b, field outlined by a dashed line for the case of $5 \mathrm{wt} . \%$ $\mathrm{H}_{2} \mathrm{O}$ ). As one can see in Fig. 17b, $P-T$ conditions for "high-pressure" $\mathrm{Hbl}$ can be simulated under nearly isobaric conditions. However, the temperatures obtained by different geothermometers are significantly different. Due to the high melt water contents, Plag crystallization is significantly suppressed, and Plag-melt equilibria gives temperatures $50-100{ }^{\circ} \mathrm{C}$ lower than those predicted by low- or high-Ca PX-melt equilibria.

In addition, although $P-T$ conditions obtained by anhydrous geothermobarometers can approach $P-T$ conditions determined earlier for $\mathrm{Hbl}$-bearing melt, the simulated high water concentrations seem unrealistic (10-12 wt.\% $\mathrm{H}_{2} \mathrm{O}$ in the most evolved dacite). The temperatures predicted by Plag-melt equilibria are always $25-50{ }^{\circ} \mathrm{C}$ lower than those predicted by other methods. These differences emphasize that the basaltic andesite melt with $5 \mathrm{wt} . \% \mathrm{H}_{2} \mathrm{O}$ at $700-900 \mathrm{MPa}$ and $1050-1100{ }^{\circ} \mathrm{C}$ was not saturated in Plag. Interestingly, a natural counterpart can be proposed for this modeled magma. Such a hydrous magma is closer to the $\mathrm{Hbl}$ stability field, and the abundant Plag-free $\mathrm{Hbl-Cpx}$ cumulates that have been observed in Plotina dome $\mathrm{Hbl}-$ andesites may represent natural counterparts of these modeled magmas. According to Fig. 17a, they can be stored in the lower half of the crust (26-30 km).

\subsubsection{Open system behavior of $\mathrm{H}_{2} \mathrm{O}$ in magma differentiation}

The results of modeling with 2 and 5 wt.\% $\mathrm{H}_{2} \mathrm{O}$ in the parental basaltic andesite lead us to the conclusion that the closed system fractionation cannot explain the crystallization sequences in the Bezymianny magma plumbing system. Below we examine the open system behavior of magma $\mathrm{H}_{2} \mathrm{O}$. To approach this goal, for every melt composition, $\mathrm{H}_{2} \mathrm{O}$ content was adjusted in order to obtain the same temperatures using both Plag-melt and Opx-melt geothermobarometers (Fig. 17b). These calculations were carried out assuming that Plag-Opx pairs coexist across a wide range of natural melts and should continuously evolve under similar conditions. As the result of the calculations, we obtained the following crystallization parameters: (1) $1100-1150{ }^{\circ} \mathrm{C}, 500-640 \mathrm{MPa}$, 1-2.5 wt.\% $\mathrm{H}_{2} \mathrm{O}$ in basaltic andesite; (2) $1130-1050{ }^{\circ} \mathrm{C}, 700-600 \mathrm{MPa}$, $2.5-5$ wt.\% $\mathrm{H}_{2} \mathrm{O}$ in $2 \mathrm{Px}$ andesite; (3) $1040-990{ }^{\circ} \mathrm{C}, 560-470 \mathrm{MPa}$, 5-6.5 wt.\% $\mathrm{H}_{2} \mathrm{O}$ in $\mathrm{Opx}$ andesite; (4) $1000{ }^{\circ} \mathrm{C}, 450-300 \mathrm{MPa}, 4.5-$ 5.5 wt.\% $\mathrm{H}_{2} \mathrm{O}$ in $\mathrm{Hbl}$ andesite Bez97-5 and $990-950{ }^{\circ} \mathrm{C}, 300-100 \mathrm{MPa}$,
3.5-5.5 wt.\% $\mathrm{H}_{2} \mathrm{O}$ in $\mathrm{Hbl}$ andesite OB-16; and (5) $900-950{ }^{\circ} \mathrm{C}$, 250-410 MPa, 6-7 wt.\% $\mathrm{H}_{2} \mathrm{O}$ in dacite.

In general, the calculated temperatures and pressures (but not melt $\mathrm{H}_{2} \mathrm{O}$ contents) resemble the results from closed system calculations. For $2 P x$ andesite OB-9 alone (open diamond in Fig. 17b) we obtained lower pressure $(300-470 \mathrm{MPa})$, higher temperature $\left(1140-1100{ }^{\circ} \mathrm{C}\right)$, and lower water content $\left(\sim 1.5\right.$ wt. $\left.\% \mathrm{H}_{2} \mathrm{O}\right)$. In contrast to another $2 \mathrm{Px}$ andesite OB-11 (gray diamond in Fig. 17b), OB-9 is richer in crystals (more phyric, $26 \%$ crystals, Table 1), more magnesian, and more (!) silica rich, with a larger modal proportion of Plag. Fig. 17b also shows that estimated conditions for $\mathrm{Hbl}$ andesite and dacite are now closer to the $P-T$ conditions field of $\mathrm{Hbl}$ stability determined using the amphibole geothermobarometer of Ridolfi et al. (2010). Based on the results, several magma evolutionary paths can be now proposed.

The conditions required for $\mathrm{Hbl}$-bearing magmas to exist can be reached via polybaric crystallization of a parental basaltic andesite with $\sim 2$ wt.\% $\mathrm{H}_{2} \mathrm{O}$ (evolutionary path I in Fig. 17b). This polybaric evolution represents a slow magma ascent from 500 to $600 \mathrm{MPa}$ depth with formation of $\mathrm{Opx}$-bearing andesites, $\mathrm{Hbl}$-bearing andesites, and finally dacites at 200-300 MPa, i.e. at depths where they could stagnate due to their high viscosity. Evolutionary path II (Fig. 17b) is responsible for the formation of $\mathrm{Hbl}$-bearing andesite with a relatively high modal proportion of Plag. It is attributed to the degassing of a closed magma system, promoting rapid crystallization due to faster decompression. This degassing mechanism is probably more important for the formation of OB-9-like 2Px andesites, characterized by a high proportion of Plag, low $\mathrm{H}_{2} \mathrm{O}$, and high $\mathrm{SiO}_{2}$ and $\mathrm{MgO}$. In this case the degassing should occur at higher $P-T$ conditions (evolutionary path III in Fig. 17b). Finally, if a hydrous $\left(\sim 5 \mathrm{wt} . \% \mathrm{H}_{2} \mathrm{O}\right)$ parental magma is stored at pressures of 600 to $900 \mathrm{MPa}$, "high-pressure" amphiboles can crystallize, and Plag-free $\mathrm{Hbl}-\mathrm{Cpx}$ assemblages ( $\mathrm{Hbl}-\mathrm{Cpx}$ cumulates) may form (evolutionary path IV in Fig. 17b).

5.6.6. Role of $\mathrm{H}_{2} \mathrm{O}-\mathrm{CO}_{2}$ rich fluids during Bezymianny magma evolution

In the last section we discuss the role of $\mathrm{H}_{2} \mathrm{O}-\mathrm{CO}_{2}$ fluid in the genesis of the Bezymianny magmas. Although we do not have any direct determinations of $\mathrm{H}_{2} \mathrm{O}$ and $\mathrm{CO}_{2}$ in the Bezymianny melts, the combined $\mathrm{H}_{2} \mathrm{O}-\mathrm{CO}_{2}$ solubility plot can be used if $P$ and $\mathrm{H}_{2} \mathrm{O}$ are known, estimating $\mathrm{CO}_{2}$ from conditions of fluid saturation at known pressure. The basic assumptions are that (1) our pressure estimates (see above) are valid, (2) magmas are fluid-saturated at these pressures, and (3) the fluids contained only $\mathrm{H}_{2} \mathrm{O}$ and $\mathrm{CO}_{2}$. Thus, the Bezymianny magma evolutionary trends I to III can be transferred from Fig. $17 \mathrm{~b}$ into $\mathrm{P}-\mathrm{H}_{2} \mathrm{O}-\mathrm{CO}_{2}$ space in Fig. 18. In Fig. 18, path I represents fluid-saturated polybaric crystallization followed by a subsequent open-system degassing (Newman and Lowenstern, 2002) during the slow ascent of the parental basaltic andesite magma. Such crystallization is accompanied by strong $\mathrm{H}_{2} \mathrm{O}$ enrichment due to much higher solubility of $\mathrm{H}_{2} \mathrm{O}$ than of $\mathrm{CO}_{2}$ in the silicate melts and results in the formation of the hydrous but nearly $\mathrm{CO}_{2}$-free dacites (path I in Fig. 18). Such $\mathrm{H}_{2} \mathrm{O}$ enrichment is consistent with estimated degrees of fractionation from incompatible trace elements (enrichment factor varies from 3 to 5 for different elements, Section 4.4). Once degassing occurs in a closed system, crystallization promotes formation of $\mathrm{Hbl}$ andesite (see path II). In contrast, fast decompression and open-system degassing (path III), with a rapid release of $\mathrm{CO}_{2}$ and a slight decrease of $\mathrm{H}_{2} \mathrm{O}$, can explain the formation of some relatively dry Plag phyric $2 \mathrm{Px}$ andesite magmas (e.g. sample OB-9).

We believe that the role of the $\mathrm{H}_{2} \mathrm{O}-\mathrm{CO}_{2}$ fluids in Bezymianny magma evolution remains to be more accurately elucidated. Due to the large uncertainty around the pressure estimates and the lack of direct information on volatile component abundances, the model presented above is a rather qualitative scheme which should be confirmed by both melt inclusions and experimental studies. One such study (Mironov and Portnyagin, 2011) has recently emphasized the importance of the decompression-degassing path for the HMB-HAB 


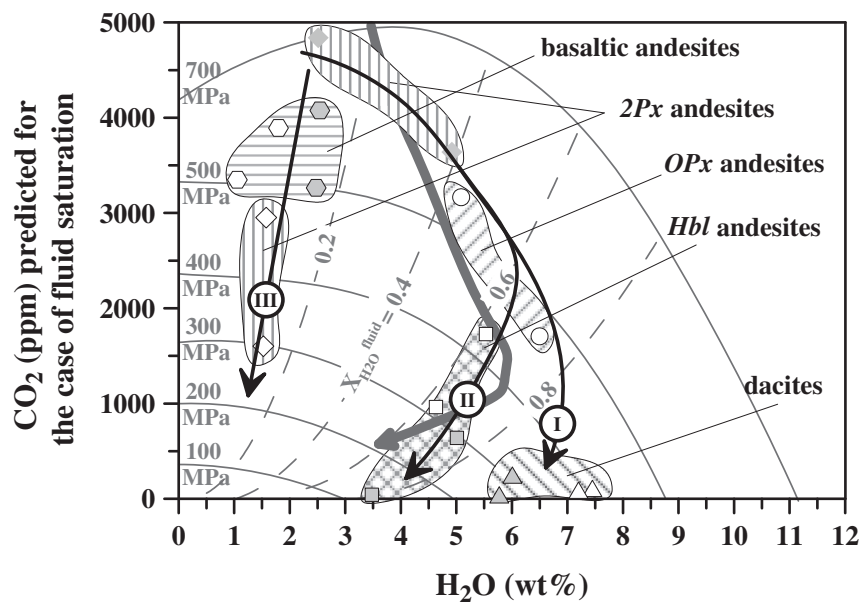

$\square-H b l$ andesite (Bez97-5)

$\square-\mathrm{Hbl}$ andesite (OB-16)

$\triangle$ - dacite (OB-40)

$\triangle$ - dacite (OB-15)

- Opx andesite (OB-42)

\section{I - Bezymianny Vocano magma evolutionary paths from Fig. 17}

$\int$

$$
\begin{aligned}
& \text { - estimated trend of the evolution of melt compositions } \\
& \text { during decompression crystallization of Kliuchevskoi } \\
& \text { Volcano magmas (Mironov and Portnyagin, 2011) }
\end{aligned}
$$

Fig. 18. Solubility plot for $\mathrm{H}_{2} \mathrm{O}$ and $\mathrm{CO}_{2}$ in basaltic melts with position of Bezymianny magmas (from basaltic andesite to dacites) and magma evolutionary paths from Fig. 17b. The solid isobars from 100 to $700 \mathrm{MPa}$ and dashed-line isopleths of constant fluid composition (in terms of $\mathrm{XH}_{2} \mathrm{O}^{\mathrm{FLUID}}$ ) for the melts saturated with $\mathrm{H}_{2} \mathrm{O}-\mathrm{CO}_{2}$-bearing fluids are from Shishkina et al. (2010) and Shishkina (2012). See text for details.

Kliuchevskoi Volcano magmas (gray thick curve in Fig. 18), which closely resembles magma evolutionary path I obtained for Bezymianny Volcano by indirect methods.

\section{Conclusions}

We interpret the trend defined by Bezymianny whole rock compositions as an LLD proxy, mostly preserved in lavas of the preBezymianny stage of volcano evolution, at the time of Raschlenionny, Dvuglavy, Plotina, and Stupenchaty lava dome formation. Later, when magma chamber(s) beneath Bezymianny started to develop and the Bezymianny edifice emerged (stages B-I to B-III), the fractional crystallization processes occurring in the developing plumbing system were continuously overlaid by additional processes of magma mixing and magma recharge in a progressively growing magma chamber. However, magmas produced in the plumbing system internally interacted and blended and finally represent cogenetic portions of different magma batches (crystal mush) that experienced crystallization along similar mineral cotectic proceeding during different periods of magma chamber evolution. Thus, magma stagnation and mixing complicates and slightly obscures the main chemical differentiation trends but does not completely erase their original LLDs. The Bezymianny plumbing system continues to evolve at present, and recently erupted andesites are produced via combined processes of (1) fractional crystallization proceeding at different levels of the plumbing system during ascent, (2) magma mixing with products of earlier differentiation stages in the chamber or in the conduit(s), and (3) inputs of new, less-differentiated magmas. Deep-seated chemical differentiation can be further complicated by the processes of (4) magma degassing and reheating at very shallow levels of the plumbing system.

Supplementary data to this article can be found online at http:// dx.doi.org/10.1016/j.jvolgeores.2013.01.003.

\section{Acknowledgments}

We thank Alexander Maksimov for sharing with us his knowledge of Bezymianny Volcano, for providing some missing samples, and for stimulating discussions. We thank Vera Ponomareva for providing dating of the Kliuchevskoi cinder cones. AR thanks the Ministry of Education of Japan for financial support of the study in 2001-2003. We gratefully acknowledge Malcolm Rutherford, Maxim Portnyagin, Francois Holtz, Roman Botcharnikov and an anonymous reviewer for their insightful comments. The editorial work of Candace O'Connor and Pavel Izbekov is greatly appreciated.

\section{References}

Adam, J., Green, T.H., 1994. The effects of pressure and temperature on the partitioning of $\mathrm{Ti}, \mathrm{Sr}$, and REE between amphibole, clinopyroxene, and basanitic melts. Chemical Geology 117 (1-4), 219-233.

Adam, J., Green, T.H., 2003. The influence of pressure, mineral composition and water on trace element partitioning between clinopyroxene, amphibole and basanitic melts. European Journal of Mineralogy 15 (5), 831-841.

Almeev, R.R., 2005. Geochemistry of Bezymianny volcano lavas: signatures of the mantle source and magma fractionation processes. PhD Thesis, Vernadsky Institute, Moscow, 238 pp.

Almeev, R.R., Ariskin, A.A., Ozerov, A.Y., Kononkova, N.N., 2002. Problems of the stoichiometry and thermobarometry of magmatic amphiboles: an example of hornblende from the andesites of Bezymianny volcano, Eastern Kamchatka. Geochemistry International 40 (8), 723-738.

Almeev, R.R., Kimura, J.-I., Ariskin, A.A., Holtz, F., Ozerov, A.Y., 2012. The role of fractional crystallization versus magma mixing in the genesis of andesites: the example of Bezymianny volcano, EMPG-XIV, Kiel, Germany, 35 pp.

Anosov, G.I., Bikenina, S.K., Popov, A.A., Sergeev, K.F., Utnasin, V.K., Fedorchenko, V.I., 1978. Deep Seismic Sounding of Kamchatka. Nauka, Moscow (129 pp., in Russian).

Arculus, R.J., 2003. Use and abuse of the terms calc-alkaline and calcalkalic. Journal of Petrology 44 (5), 929-935.

Ariskin, A.A., 1999. Phase equilibria modeling in igneous petrology: use of COMAGMAT model for simulating fractionation of ferro-basaltic magmas and the genesis of high-alumina basalt. Journal of Volcanology and Geothermal Research 90, 115-162.

Ariskin, A.A., Barmina, G.S., Ozerov, A.Y., Nielsen, R.L., 1995. Genesis of high-alumina basalts from Klyuchevskoy volcano. Petrology 3, 449-472.

Auer, S., Bindeman, I., Wallace, P., Ponomareva, V., Portnyagin, M., 2009. The origin of hydrous, high- $\delta^{18} \mathrm{O}$ voluminous volcanism: diverse oxygen isotope values and high magmatic water contents within the volcanic record of Klyuchevskoy volcano, Kamchatka, Russia. Contributions to Mineralogy and Petrology 157 (2), 209-230.

Baker, M.B., Grove, T.L., Price, R., 1994. Primitive basalts and andesites from the Mt. Shasta region, N. California - products of varying melt fraction and watercontent. Contributions to Mineralogy and Petrology 118 (2),111-129.

Baranov, B.V., Seliverstov, N.I., Murav'ev, A.V., Muzurov, E.L., 1991. The Komandorsky Basin as a product of spreading behind a transform plate boundary. Tectonophysics 199, 237-270.

Beard, J.S., Lofgren, G.E., 1991. Dehydration melting and water-saturated melting of basaltic and andesitic greenstones and amphibolites at 1, 3, and $6.9 \mathrm{~kb}$. Journal of Petrology 32 (2), 365-401.

Bedard, J.H., 2006. Trace element partitioning in plagioclase feldspar. Geochimica et Cosmochimica Acta 70 (14), 3717-3742.

Belousov, A., 1996. Deposits of 30 March 1956 directed blast at Bezymianny volcano, Kamchatka, Russia. Bulletin of Volcanology 57, 649-662.

Belousov, A., Voight, B., Belousova, M., Petukhin, A., 2002. Pyroclastic surges and flows from the 8-10 May 1997 explosive eruption of Bezymianny volcano, Kamchatka, Russia. Bulletin of Volcanology 64 (7), 455-471.

Blatter, D.L., Carmichael, I.S.E., 2001. Hydrous phase equilibria of a Mexican high-silica andesite: a candidate for a mantle origin? Geochimica et Cosmochimica Acta 65 (21), 4043-4065

Bogoyavlenskaya, G.E., Braitseva, O.A., Melekestsev, I.V., Kiriyanov, V.Y., Miller, C.D., 1985. Catastrophic eruptions of the directed-blast type at Mount St. Helens, Bezymianny and Shiveluch volcanoes. Journal of Geodynamics 3 (3-4), 189-218.

Bogoyavlenskaya, G.Y., Braitseva, O.A., Melekestsev, I.V., Maksimov, A.P., Ivanov, B.V., 1991. Bezymianny volcano. In: Fedotov, S.A., Masurenkov, Y.P. (Eds.), Active Volcanoes of Kamchatka. Nauka, Moscow, pp. 195-197.

Botcharnikov, R.E., Almeev, R.R., Koepke, J., Holtz, F., 2008. Experimental phase relations, mineral-melt equilibria and liquid lines of descent in a hydrous ferrobasalt implications for the Skaergaard intrusion and Columbia River flood basalts. Journal of Petrology 49 (9), 1687-1727.

Braitseva, O.A., Melekestsev, I.V., Bogoyavlenskaya, G.E., Maksimov, A.P., 1991. Bezymianny: eruptive history and dynamics. Volcanology and Seismology 12, 165-195.

Braitseva, O.A., Melekestsev, I.V., Ponomareva, V.V., Sulerzhitsky, L.D., 1995. The ages of calderas, large explosive craters and active volcanoes in the Kuril-Kamchatka region, Russia. Bulletin of Volcanology 57 (6), 383-402. 
Brophy, J.G., 1986. The Cold Bay volcanic center, Aleutian volcanic arc:1. implications for the origin of high-alumina arc basalt. Contributions to Mineralogy and Petrology 93 (3), 368-380.

Brophy, J.G., 1987. The Cold Bay volcanic center, Aleutian volcanic arc: 2. Implications for fractionation and mixing mechanism in calc-alkaline genesis. Contributions to Mineralogy and Petrology 97, 378-388.

Brophy, J.G., 1989. Can high-alumina arc basalt be derived from low-alumina arc basalt evidence from Kanaga Island, Aleutian Arc, Alaska. Geology 17 (4), 333-336.

Brophy, J., 2008. A study of rare earth element (REE) $-\mathrm{SiO}_{2}$ variations in felsic liquids generated by basalt fractionation and amphibolite melting: a potential test for discriminating between the two different processes. Contributions to Mineralogy and Petrology 156 (3), 337-357.

Churikova, T., Dorendorf, F., Wörner, G., 2001. Sources and fluids in the mantle wedge below Kamchatka, evidence from across-arc geochemical variation. Journal of Petrology 42 (8), 1567-1593.

Conte, A.M., Palladino, D.M., Perinelli, C., Argenti, E., 2010. Petrogenesis of the highalumina basalt-andesite suite from Sant'Antioco Island, SW Sardinia, Italy. Periodico Di Mineralogia 79 (1), 27-55.

Crawford, A.J., Falloon, T.J., Eggins, S., 1987. The origin of island arc high-alumina basalts. Contributions to Mineralogy and Petrology 97, 417-430.

Davidson, J., Turner, S., Handley, H., Macpherson, C., Dosseto, A., 2007. Amphibole "sponge" in arc crust? Geology 35 (9), 787-790.

Defant, M.J., Drummond, M.S., 1990. Derivation of some modern arc magmas by melting of young subducted lithosphere. Nature 347, 662-665.

Dobosi, G., Jenner, G.A., 1999. Petrologic implications of trace element variation in clinopyroxene megacrysts from the Nograd volcanic province, north Hungary: a study by laser ablation microprobe-inductively coupled plasma-mass spectrometry. Lithos 46 (4), 731-749.

Dorendorf, F., Wiechert, U., Wörner, G., 2000. Hydrated sub-arc mantle: a source for the Klyuchevskoy volcano, Kamchatka/Russia. Earth and Planetary Science Letters 175 $(1-2), 69-86$.

Dosseto, A., Bourdon, B., Joron, J.L., Dupre, B., 2003. U-Th-Pa-Ra study of the Kamchatka arc: new constraints on the genesis of arc lavas. Geochimica et Cosmochimica Acta 67 (15), 2857-2877.

Eichelberger, J.C., 1978. Andesitic volcanism and crustal evolution. Nature 275, 21-27.

Eichelberger, J.C., Izbekov, P.E., Browne, B.L., 2006. Bulk chemical trends at arc volcanoes are not liquid lines of descent. Lithos 87 (1-2), 135-154.

Ermakov, V.A., 1977. Associations of Quaternary Volcanic Rocks. Nedra, Moscow (223 pp.).

Fedotov, S.A., Zharinov, N.A., Gorel'chik, V.I., 1988. Deformation and earthquakes of Klyuchevskoy volcano: a model of its activity. Volcanology and Seismology 2, 3-42.

Fedotov, S.A., Masurenkov, Y.P., Balesta, S.T., Gushchenko, I.I., Kozhemyaka, N.N., Svyatlovsky, A.Y., Khrenov, A.P., Chirkov, A.M., Yanshina, F.T., Shutova, G.S., 1991. Active Volcanoes of Kamchatka. Nauka, Moscow (302 pp.).

Geist, E.L., Scholl, D.W., 1994. Large-scale deformation related to the collision of the Aleutian Arc with Kamchatka. Tectonics 13, 538-560.

Gertisser, R., Keller, J., 2000. From basalt to dacite: origin and evolution of the calcalkaline series of Salina, Aeolian Arc, Italy. Contributions to Mineralogy and Petrology 139 (5), 607-626.

Gill, J.B., 1981. Orogenic andesite and plate tectonics. Springer-Verlag, Berlin, Heidelberg, New York (385 pp.).

Gorbatov, A., Kostoglodov, V., Suarez, G., Gordeev, E.I., 1997. Seismicity and structure of the Kamchatka subduction zone. Journal of Geophysical Research 102 (B8), 17883-17898.

Gordeev, E.I. (Ed.), 2006. Problems of the explosive volcanism (dedicated to the 50th year of the catastrophic eruption of Bezymianny volcano), PetropavlovskKamchatsky, p. 186 (in Russian).

Gorshkov, G.S., 1959. Gigantic eruption of the volcano Bezymianny. Bulletin of Volcanology 20 (1), 77-109.

Gorshkov, G.S., Bogoyavlenskaya, G.E., 1965. Bezymianny volcano and specifics of its most recent eruption in 1955-1963. Nauka, Moscow (170 pp.)

Green, D.H., 1973. Contrasted Melting Relations in a Pyrolite Upper Mantle under MidOceanic Ridge, Stable Crust and Island Arc Environments. Tectonophysics 17 (3), 285-297.

Green, T.H., 1994. Experimental studies of trace-element partitioning applicable to igneous petrogenesis - Sedona 16 years later. Chemical Geology 117 (1-4), 1-36.

Green, T.H., Blundy, J.D., Adam, J., Yaxley, G.M., 2000. SIMS determination of trace element partition coefficients between garnet, clinopyroxene and hydrous basaltic liquids at 2-7.5 GPa and $1080-1200{ }^{\circ} \mathrm{C}$. Lithos 53 (3-4), 165-187.

Grove, T.L., 1993. Corrections to expressions for calculating mineral components in "Origin of calc-alkaline series lavas at Medicine Lake volcano by fractionation, assimilation and mixing" and "Experimental petrology of normal MORB near the Kane Fracture Zone: $22^{\circ}-25^{\circ} \mathrm{N}$, mid-Atlantic ridge". Contributions to Mineralogy and Petrology 114 (3), 422-424.

Grove, T.L., Baker, M.B., 1984. Phase equilibrium controls on the tholeiitic versus calcalkaline differentiation trends. Journal of Geophysical Research 89 (B5), 3253-3274.

Grove, T.L., Kinzler, R.J., 1986. Petrogenesis of andesites. Annual Review of Earth and Planetary Sciences 14, 417-454.

Grove, T.L., Gerlach, D.C., Sando, T.W., 1982. Origin of calc-alkaline series lavas at Medicine Lake volcano by fractionation, assimilation and mixing. Contributions to Mineralogy and Petrology 80 (2), 160-181.

Grove, T.L., Elkins-Tanton, L.T., Parman, S.W., Chatterjee, N., Muntener, O., Gaetani, G.A., 2003. Fractional crystallization and mantle-melting controls on calcalkaline differentiation trends. Contributions to Mineralogy and Petrology 145 (5), 515-533.

Hamada, M., Fujii, T., 2007. $\mathrm{H}_{2} \mathrm{O}$-rich island arc low-K tholeiite magma inferred from Carich plagioclase-melt inclusion equilibria. Geochemical Journal 41 (6), 437-461.
Hart, S.R., Dunn, T., 1993. Experimental cpx/melt partitioning of 24 trace elements. Contributions to Mineralogy and Petrology 113, 1-8.

Heath, E., Macdonald, R., Belkin, H., Hawkesworth, C., Sigurdsson, H., 1998. Magmagenesis at Soufriere Volcano, St Vincent, Lesser Antilles Arc. Journal of Petrology 39 (10), 1721-1764.

Herd, C.D.K., Treiman, A.H., McKay, G.A., Shearer, C.K., 2004. The behavior of Li and B during planetary basalt crystallization. American Mineralogist 89 (5-6), 832-840.

Irvine, T.N., Baragar, W.R., 1971. A guide to the chemical classification of the common igneous rocks. Canadian Journal of Earth Sciences 8 (523-548).

Ivanov, B.V., 1990. Types of andesitic volcanism of the Pacific active zone. Nauka Moscow (213 pp.)

Kadik, A.A., Maksimov, A.P., Ivanov, B.V., 1986. Physico-chemical conditions of crystallization and genesis of andesites. Nauka, Moscow (158 pp.).

Kawamoto, T., 1996. Experimental constraints on differentiation and $\mathrm{H}_{2} \mathrm{O}$ abundance of calc-alkaline magmas. Earth and Planetary Science Letters 144, 577-589.

Kelley, K.A., Cottrell, E., 2009. Water and the Oxidation State of Subduction Zone Magmas. Science 325 (5940), 605-607.

Kepezhinskas, P., McDermont, F., Defant, M.J., Hochstaedter, A., Drummod, M.S. Hawkesworth, C.J., Koloskov, A., Maury, R.C., Bellon, H., 1997. Trace element and $\mathrm{Sr}-\mathrm{Nd}-\mathrm{Pb}$ isotopic constraints on a three-component model of Kamchatka arc petrogenesis. Geochimica et Cosmochimica Acta 61 (3), 577-600.

Kersting, A.B., 1995. $\mathrm{Pb}$ isotope ratios of north pacific sediments, sites 881, 883, and 884: Implications for sediment recycling in the Kamchatka arc. Proceedings of the Ocean Drilling Program, Scientific Results 145, 383-388.

Kersting, A.B., Arculus, R.J., 1994. Klyuchevskoy volcano, Kamchatka, Russia: the role of high-flux recharged, tapped, and fractionated magma chamber(s) in the genesis of high- $\mathrm{Al}_{2} \mathrm{O}_{3}$ from high-MgO basalt. Journal of Petrology 35 (1), 1-41.

Khrenov, A.P., Dvigalo, V.N., Kirsanov, I.T., Fedotov, S.A., Gorel'chik, V.I., Zharinov, N.A., 1991. Klyuchevskoy volcano. In: Fedotov, S.A., Masurenkov, Y.P. (Eds.), Active volcanoes of Kamchatka. Nauka, Moscow, pp. 146-153.

Khubunaya, S.A., Bogoyavlenskii, S.O., Novgorodtseva, T.Y., Okrugina, A.I., 1993. Mineralogical features of magnesian basalts: evidence of fractionation in the magmatic chamber of Klyuchevskoy volcano. Volcanology and Seismology (3), 46-68.

Khubunaya, S.A., Gontovaya, L.I., Sobolev, A.V., Nizcous, I.V., 2007. Magma chambers beneath Klyuchevskoy Group of volcanoes (Kamchatka). Journal of Volcanology and Seismology 1 (2), 98-118.

Kinzler, R.J., Grove, T.L., Recca, S.I., 1990. An experimental study on the effect of temperature and melt composition on the partitioning of nickel between olivine and silicate melt. Geochimica et Cosmochimica Acta 54 (5), 1255-1265.

Koulakov, I., Gordeev, E.I., Dobretsov, N.L., Vernikovsky, V.A., Senyukov, S., Jakovlev, A 2011. Feeding volcanoes of the Kluchevskoy group from the results of local earthquake tomography. Geophysical Research Letters 38 (9), L09305.

Kushiro, I., 1969. The system forsterite-diopside-silica with and without water at high pressures. American Journal of Science 267A, 269-294.

Le Bas, M.J., Le Maitre, R.W., Streckeisen, A., Zanettin, B., 1986. A chemical classification of volcanic rocks based on the total alkali-silica diagram. Journal of Petrology 27, 745-750.

Le Maitre, R.W., Bateman, P., Dudek, A., Keller, J., Lameyre, J., Le Bas, M.J., Sabine, P.A Schmid, R., Sorensen, H., Streckeisen, A., Woolley, A.R.Z.B., 1989. A Classification of Igneous Rocks and Glossary of Terms: Recommendations of the International Union of Geological Sciences Subcommission on the Systematics of Igneous Rocks. Blackwell Scientific, Oxford.

Lees, J.M., Symons, N., Chubarova, O., Gorelchik, V., Ozerov, A.Y., 2007. Tomographic Images of Klyuchevskoy Volcano P-wave Velocity. In: Eichelberger, J.C., Gordeev, E.I., Kasahara, M., Izbekov, P., Lees, J. (Eds.), Volcanism and Subduction: The Kamchatka Region Geophysical Monograph Series. American Geophysical Union, Washington, pp. 297-306.

Lepage, L.D., 2003. ILMAT: an Excel worksheet for ilmenite-magnetite geothermometry and geobarometry. Computers \& Geosciences 29 (5), 673-678.

Levin, V., Shapiro, N., Park, J., Ritzwoller, M., 2002. Seismic evidence for catastrophic slab loss beneath Kamchatka. Nature 418 (6899), 763-767.

Luhr, J.F., Carmichael, I.S.E., 1980. The Colima volcanic complex, Mexico: 1. Post-caldera andesites from Volcan Colima. Contributions to Mineralogy and Petrology 71 (4), 343-372.

Maksimov, A.P., Kadik, A.A., Korovushkina, E.Y., Ivanov, B.V., 1978. Crystallization of an andesite melt with a fixed water content at pressures up to 12 kbar. Geochemistry International 15, 20-29.

Martel, C., Pichavant, M., Holtz, F., Scaillet, B., 1999. Effects of $\mathrm{fO}_{2}$ and $\mathrm{H}_{2} \mathrm{O}$ on andesite phase relations between 2 and 4 kbar. Journal of Geophysical Research 104 (B12), 29453-29470.

Mironov, N.L., Portnyagin, M.V., 2011. $\mathrm{H}_{2} \mathrm{O}$ and $\mathrm{CO}_{2}$ in parental magmas of Kliuchevskoi volcano inferred from study of melt and fluid inclusions in olivine. Russian Geology and Geophysics 52 (11), 1353-1367.

Mironov, N.L., Portnyagin, M., Plechov, P.Y., Khubunaya, C.A., 2001. Final stages of magma evolution in Klyuchevskoy volcano, Kamchatka: evidence from melt Inclusions in minerals of high-alumina basalts. Petrology 9 (1), 46-62.

Miyashiro, A., 1974. Volcanic rock series in island arcs and active continental margins. American Journal of Science 274, 321-355.

Moore, G., Carmichael, I.S.E., 1998. The hydrous phase equilibria (to $3 \mathrm{kbar}$ ) of an andesite and basaltic andesite from western Mexico: constraints on water conten and conditions of phenocryst growth. Contributions to Mineralogy and Petrology 130, 304-319.

Münker, C., Wörner, G. Yogodzinski, G.M., Churikova, T., 2004. Behaviour of high field strength elements in subduction zones: constraints from Kamchatka-Aleutian arc lavas. Earth and Planetary Science Letters 224 (3-4), 275-293.

Newman, S., Lowenstern, J.B., 2002. VolatileCalc: a silicate melt- $\mathrm{H}_{2} \mathrm{O}-\mathrm{CO}_{2}$ solution model written in Visual Basic for Excel. Computers \& Geosciences 28 (5), 597-604. 
Nye, C.J., Reid, M.R., 1986. Geochemistry of primary and least fractionated lavas from Okmok volcano, central Aleutians: implications for arc magma genesis. Journal of Geophysical Research 91, 10271-10287.

Ozerov, A.Y., 2000. The evolution of high-alumina basalts of the Klyuchevskoy volcano, Kamchatka, Russia, based on microprobe analyses of mineral inclusions. Journal of Volcanology and Geothermal Research 95 (1-4), 65-79.

Ozerov, A.A., Ariskin, A.A., Kyle, P., Bogoyavlenskaya, G.E., Karpenko, S.F., 1997. Petrological-geochemical model for genetic relationships between basaltic and andesitic magmatism of Klyuchevskoy and Bezymianny volcanoes, Kamchatka. Petrology 5 (6), 550-569.

Pichavant, M., Macdonald, R., 2007. Crystallization of primitive basaltic magmas at crustal pressures and genesis of the calc-alkaline igneous suite: experimental evidence from St Vincent, Lesser Antilles arc. Contributions to Mineralogy and Petrology 154 (5), 535-558.

Pichavant, M., Martel, C., Bourdier, J.-L, Scaillet, B., 2002. Physical conditions, structure and dynamics of a zoned magma chamber: Mount Pelee (Martinique, Lesser Antilles Arc). Journal of Geophysical Research 107 (B5), 1-26.

Pineau, F., Semet, M.P., Grassineaua, N., Okrugin, V.M., Javoy, M., 1999. The genesis of the stable isotope $(\mathrm{O}, \mathrm{H})$ record in arc magmas: the Kamtchatka's case. Chemical Geology 153 (1-4), 93-124.

Plechov, P.Y., Mironov, N.L., Plechova, A.A., Khubunaya, S.A., 2000. Compositional peculiarities and genesis of melt inclusions in plagioclase from the Apakhonchich lava flow, Klyuchevskoy volcano, Kamchatka. Geochemistry International 1, 34-41.

Plechov, P., Tsai, A., Shcherbakov, V., Dirksen, O., 2008. Opacitization conditions of hornblende in Bezymyannyi volcano andesites (March 30, 1956 eruption). Petrology $16(1), 19-35$.

Portnyagin, M., Manea, V.C., 2008. Mantle temperature control on composition of arc magmas along the Central Kamchatka Depression. Geology 36 (7), 519-522.

Portnyagin, M., Hoernle, K., Avdeiko, G., Hauff, F., Werner, R., Bindeman, I., Uspensky, V., Garbe-Schonberg, D., 2005. Transition from arc to oceanic magmatism at the Kamchatka-Aleutian junction. Geology 33 (1), 25-28.

Portnyagin, M., Hoernle, K., Plechov, P., Mironov, N., Khubunaya, S., 2007a. Constraint on mantle melting and composition and nature of slab components in volcanic arcs from volatiles ( $\mathrm{H} 2 \mathrm{O}, \mathrm{S}, \mathrm{Cl}, \mathrm{F})$ and trace elements in melt inclusions from the Kamchatka Arc. Earth and Planetary Science Letters 255 (1-2), 53-69.

Portnyagin, M., Bindeman, I.N., Hoernle, K., Hauff, F., 2007b. Geochemistry of primitive lavas of the central Kamchatka Depression: magma generation at the edge of the Pacific plate. In: Eichelberger, J.C., Gordeev, E.I., Kasahara, M., Izbekov, P., Lees, J. (Eds.), Volcanism and Subduction: The Kamchatka Region Geophysical Monograph Series. American Geophysical Union, Washington, pp. 203-244.

Putirka, K.D., 2008. Thermometers and barometers for volcanic systems. Reviews in Mineralogy and Geochemistry 69 (1), 61-120.

Reubi, O., Blundy, J., 2009. A dearth of intermediate melts at subduction zone volcanoes and the petrogenesis of arc andesites. Nature 461 (7268), 1269-1273.

Ridolfi, F., Renzulli, A., Puerini, M., 2010. Stability and chemical equilibrium of amphibole in calc-alkaline magmas: an overview, new thermobarometric formulation and application to subduction-related volcanoes. Contributions to Mineralogy and Petrology 160 (1), 45-66.

Righter, K., Leeman, W.P., Hervig, R.L., 2006. Partitioning of Ni, Co and V between spinel-structured oxides and silicate melts: importance of spinel composition. Chemical Geology 227 (1-2), 1-25.

Sakuyama, M., 1981. Petrological study of the Myoko and Kurohime volcanoes, Japan: crystallization sequence and evidence for magma mixing. Journal of Petrology 22, 553-583.

Shcherbakov, V., Plechov, P., Izbekov, P., Shipman, J., 2011. Plagioclase zoning as an indicator of magma processes at Bezymianny Volcano, Kamchatka. Contributions to Mineralogy and Petrology 162 (1), 83-99.

Shishkina, T.A., 2012. Storage conditions and degassing processes of low-K and high-A tholeiitic island-arc magmas: Experimental constraints and natural observations for Mutnovsky volcano, Kamchatka. PhD Thesis, Leibniz University of Hannover, Hannover, $214 \mathrm{pp}$.

Shishkina, T., Botcharnikov, R., Holtz, F., Almeev, R.R., Portnyagin, M., 2010. Solubility of $\mathrm{H}_{2} \mathrm{O}$ and $\mathrm{CO}_{2}$-bearing fluids in tholeiitic basalts at pressures up to $500 \mathrm{MPa}$. Chemical Geology 277 (1-2), 115-125.
Sisson, T.W., 1994. Hornblende-melt trace element partitioning measured by ion microprobe. Chemical Geology 117, 331-344.

Sisson, T.W., Grove, T.L., 1993. Experimental investigations of the role of $\mathrm{H}_{2} \mathrm{O}$ in calcalkaline differentiation and subduction zone magmatism. Contributions to Mineralogy and Petrology 113 (2), 143-166.

Smith, T.E., Thirlwall, M., Holm, P.E., Harris, M.J., 2004. Petrogenesis of orthopyroxeneand amphibole-bearing andesites, Mustique, Grenadine Islands, Lesser Antilles Arc: isotope, trace element and physical constraints. Island Arc 13 (1), 73-94.

Spencer, K.J., Lindsley, D.H., 1981. A solution model for coexisting iron-titanium oxides. American Mineralogist 66 (11/12), 1189-1201.

Stormer, J.C.J., 1983. The effects of recalculation on estimates of temperature and oxygen fugacity from analyses of multicomponent iron-titanium oxides. American Mineralogist 68, 586-594.

Sun, S.-S., McDonough, W.F., 1989. Chemical and isotopic systematics of oceanic basalts: implications for mantle composition and processes. In: Saunders, A.D. Norry, M.J. (Eds.), Magmatism in the Ocean Basins. Geological Society London Special Publications, London, pp. 313-345.

Tatsumi, Y., Kogiso, T., 2003. The subduction factory: its role in the evolution of Earth's crust and mantle. In: Larter, R.D., Leat, P.T. (Eds.), Intra-Oceanic Subduction Systems: Tectonic and Magmatic Processes. Geological Society Special Publication. Geological Society of London, London, pp. 55-80.

Tatsumi, Y., Takahashi, E., 2006. Operation of subduction factory and production of andesite. Journal of Mineralogical and Petrological Sciences 101 (3), 145-153.

Tatsumi, Y., Kogiso, T., Nohda, S., 1995. Formation of a third volcanic chain in Kamchatka: generation of unusual subduction-related magmas. Contributions to Mineralogy and Petrology 120 (2), 117-128.

Taylor, S.R., Capp, A.C., Graham, A.L., Blake, D.H., 1969. Trace element abundances in andesites. Contributions to Mineralogy and Petrology 23 (1), 1-26.

Thelen, W., West, M., Senyukov, S., 2010. Seismic characterization of the fall 2007 eruptive sequence at Bezymianny Volcano, Russia. Journal of Volcanology and Geothermal Research 194 (4), 201-213.

Thirlwall, M.F., G. A.M., Arculus, R.J., Harmon, R.S., MacPherson, C.G., 1996. Resolution of the effects of crustal assimilation, sediment subduction, and fluid transport in island arc magmas: $\mathrm{Pb}-\mathrm{Sr}-\mathrm{Nd}-\mathrm{O}$ isotope geochemistry of Grenada, Lesser Antilles. Geochimica et Cosmochimica Acta 60 (23), 4785-4810.

Tolstykh, M.L, Naumov, V.B., Bogoyavlenskaya, G.E., Kononkova, N.N., 1999. The role of andesitic-rhyolitic-dacitic melts in the crystallization of phenocrysts in andesite of Bezymyannyi Volcano, Kamchatka. Geokhimiya (1), 14-24.

Tormey, D.R., Grove, T.L., Bryan, W.B., 1987. Experimental petrology of normal MORB near the Kane Fracture Zone: $22^{\circ}-25^{\circ} \mathrm{N}$, mid-Atlantic ridge. Contributions to Mineralogy and Petrology 96 (1), 121-139.

Turner, S., McDermott, F., Hawkesworth, C.L., Kepezhinskas, P., 1998. A U-series study of lavas from Kamchatka and the Aleutians: constraints on source composition and melting processes. Contributions to Mineralogy and Petrology 133 (3), 217-234.

Turner, S., Sims, K.W.W., Reagan, M., Cook, C., 2007. A ${ }^{210} \mathrm{~Pb}-{ }^{226} \mathrm{Ra}-{ }^{230} \mathrm{Th}-{ }^{238} \mathrm{U}$ study of Klyuchevskoy and Bezymianny volcanoes, Kamchatka. Geochimica et Cosmochimica Acta 71 (19), 4771-4785.

Utnasin, V.K., Abdurakhmanov, A.L., Anosov, G.I., Budyansky, Y.A., Fedorchenko, V.I., Markhinin, Y.K., Balesta, S.T., 1976. Types of magma foci of island arc volcanoes and their study by the method of deep seismic sounding in Kamchatka. In: Aoki, H., Iizuka, S. (Eds.), Volcanoes and Tectonosphere. Tokai University Press, pp. 123-137.

Wilson, M., 1989. Igneous Petrogenesis.Kluwer, Dordrecht (466 pp.).

Wood, B.J., Turner, S.P., 2009. Origin of primitive high-Mg andesite: constraints from natural examples and experiments. Earth and Planetary Science Letters 283 (1-4), 59-66.

Yang, H.J., Kinzler, R.J., Grove, T.L., 1996. Experiments and models of anhydrous, basaltic olivine-plagioclase-augite saturated melts from 0.001 to $10 \mathrm{kbar}$. Contributions to Mineralogy and Petrology 124 (1), 1963-1973.

Yoder, H.S., Tilley, C.E., 1962. Origin of basaltic magma: an experimental study of natural and synthetic rock systems. Journal of Petrology 3 (3), 342-532.

Yogodzinski, G.M., Lees, J.M., Churikova, T.G., Dorendorf, F., Woerner, G., Volynets, O.N. 2001. Geochemical evidence for the melting of subducting oceanic lithosphere at plate edges. Nature 404, 500-504 